Florida International University FIU Digital Commons

FIU Electronic Theses and Dissertations

University Graduate School

3-29-2012

\title{
The US Financial Crisis and the Behavior of the Foreign Exchange Market
}

Chaiyuth Padungsaksawasdi

Florida International University, cpadu001@fiu.edu

DOI: $10.25148 /$ etd.FI12050246

Follow this and additional works at: https://digitalcommons.fiu.edu/etd

\section{Recommended Citation}

Padungsaksawasdi, Chaiyuth, "The US Financial Crisis and the Behavior of the Foreign Exchange Market" (2012). FIU Electronic Theses and Dissertations. 642.

https://digitalcommons.fiu.edu/etd/642

This work is brought to you for free and open access by the University Graduate School at FIU Digital Commons. It has been accepted for inclusion in FIU Electronic Theses and Dissertations by an authorized administrator of FIU Digital Commons. For more information, please contact dcc@fiu.edu. 


\title{
FLORIDA INTERNATIONAL UNIVERSITY \\ Miami, Florida
}

\section{THE US FINANCIAL CRISIS AND THE BEHAVIOR OF THE FOREIGN EXCHANGE MARKET}

\author{
A dissertation submitted in partial fulfillment of the \\ requirements for the degree of \\ DOCTOR OF PHILOSOPHY \\ in \\ BUSINESS ADMINISTRATION \\ By
}

Chaiyuth Padungsaksawasdi

2012 
To: Dean Joyce Elam

College of Business Administration

This dissertation, written by Chaiyuth Padungsaksawasdi, and entitled The US Financial Crisis and the Behavior of the Foreign Exchange Market, having been approved in respect to style and intellectual content, is referred to you for judgment.

We have read this dissertation and recommend that it be approved.

$\begin{array}{r}\hline \text { Gauri Ghai } \\ \hline \text { Arun J. Prakash } \\ \hline \text { Ali M. Parhizgari, Major Professor }\end{array}$

Date of Defense: March 29, 2012

The dissertation of Chaiyuth Padungsaksawasdi is approved.

Dean Joyce Elam

College of Business Administration

Dean Lakshmi N. Reddi

University Graduate School

Florida International University, 2012 
(C) Copyright 2012 by Chaiyuth Padungsaksawasdi All rights reserved. 


\section{ACKNOWLEDGMENTS}

First and foremost, I wish to acknowledge the assistance, guidance, support, and direction I received from my dissertation chair, Dr. Ali M. Parhizgari, in conducting this research. Without his enthusiastic support and encouragement, this dissertation would not have been possible.

I am grateful to my doctoral committee members, Dr. Arun J. Prakash, Dr. Suchismita Mishra, and Dr. Gauri Ghai for their support and guidance. I am also grateful to the faculty and staff of the Department of Finance and Real Estate for their support and encouragement throughout my Ph.D. studies.

Last, but not least, I wish to acknowledge my family and friends for all their support, patience, understanding, and love. I am very grateful to my parents, who shared with me the pains and pleasures of my doctoral studies at Florida International University and helped to make it a memorable and worthwhile experience. 


\section{ABSTRACT OF THE DISSERTATION}

\section{THE US FINANCIAL CRISIS AND THE BEHAVIOR OF THE FOREIGN}

\section{EXCHANGE MARKET}

by

Chaiyuth Padungsaksawasdi

Florida International University, 2012

Miami, Florida

\section{Professor Ali M. Parhizgari, Major Professor}

Foreign exchange market is the most active market in today's global financial domains. While the consensus on several aspects of this market is fairly established, the informational efficiency in this market is still unsettled, particularly during unexpected interruptions and unusual or unstable periods. The financial crisis of 2008 is the most recent example of such a period.

This dissertation focuses on the efficiency of the foreign exchange market during a unique, turbulent period using the six most actively traded currencies: the Australian dollar, Canadian dollar, Swiss franc, Euro, British pound, and Japanese yen. Considering nine months before the peak of the financial crisis to nine months thereafter, the entire sample is divided into three sub-samples: full-, non-crisis-, and crisis-periods. Both daily and minute-by-minute data are used. A variety of instruments are analyzed, including spot, forward, and exchange traded funds on the currencies. The methodologies that are employed range from standard econometric tests of efficiency to estimation of vector error correction models to identify price discovery, or leadership positions, in each of the currency markets. 
The findings indicate behavioral similarities and differences. The patterns of the volatility of the currencies are mixed: two-humped for the AUD, CAD, and EUR; Wshaped for the CHF; three-humped for the GBP, and flat U-shaped for the JPY. The daily results from several methodologies provide mixed evidence on market efficiency. Over the entire sample period, the estimated forward premium coefficients from the GARCH $(1,1)$ model are not significant for all currencies, while the null hypotheses of zero and one cointegrating vectors cannot be rejected for all currencies, except for the AUD. These findings are consistent with some of the previous studies, concluding that the efficiency tests in the foreign exchange market would depend on the methodology and the time period of the study.

The high frequency data results show different degrees of price discovery between pair-wise instruments. Specifically, the spot exchange market shows a greater contribution to price discovery than the corresponding exchange traded funds. A possible explanation is the current size of the market and its increased transparency through the use of electronic trading. 


\section{TABLE OF CONTENTS}

CHAPTER

PAGE

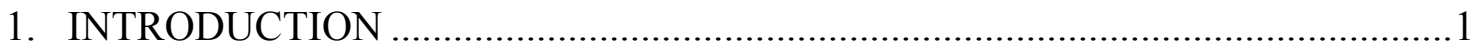

1.1. Motivation and Purpose ..........................................................................

1.2. Background ..............................................................................................

1.3. Prior Literature on the Efficiency of the Foreign Exchange Markets ..................

1.4. Objectives ..................................................................................................

2. FUNDAMENTAL ASPECTS OF THE BEHAVIOR OF THE FOREX MARKET

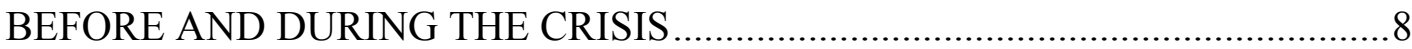

2.1. Data Description and the Determination of the Periods ......................................

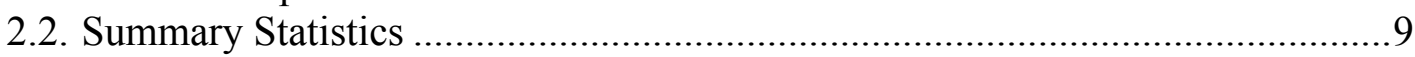

2.3. Tests of Equality of Means Between the Non-Crisis and Crisis Periods ...........13

2.4. Day-of-the-Week Effect .................................................................................. 14

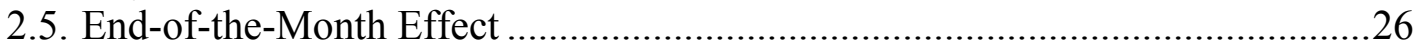

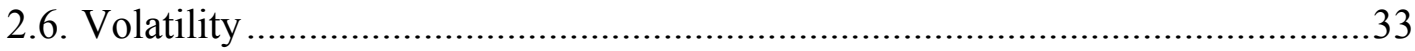

2.6.1. Volatility Pattern on Day-of-the-Week ...................................................33

2.6.2. Volatility Pattern on End-of-the-Month ....................................................37

2.7. Summary and Conclusions ............................................................................ 39

3. EFFICIENCY OF THE FOREX MARKET BEFORE AND DURING THE CRISIS

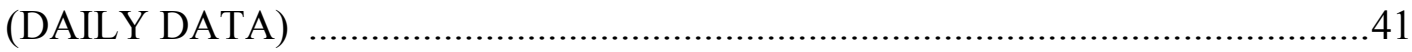

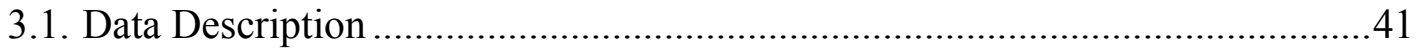

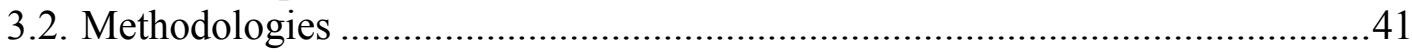

3.2.1. Forward Rate as an Unbiased Predictor of Future Spot Rate ...................42

3.2.2. GARCH $(1,1)$ Specification Model ..........................................................43

3.2.3. Dummy Variable Models ....................................................................4

3.2.4. Cointegration Method.........................................................................45

3.3. Empirical Results ......................................................................................

3.3.1. Regression Tests of Forward Rate as an Unbiased Predictor of Future

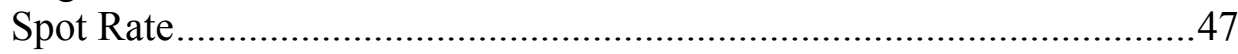

3.3.2. GARCH $(1,1)$ Specification Model .........................................................54

3.3.3. Tests of Equality of Estimated Intercepts and Coefficients Between the Non-Crisis and Crisis Periods of the GARCH $(1,1)$ Model .....................57

3.3.4. Bivariate Cointegration Analysis..........................................................58

3.4. Summary and Conclusions .........................................................................64

4. EFFICIENCY OF THE FOREX MARKET BEFORE AND DURING THE CRISIS

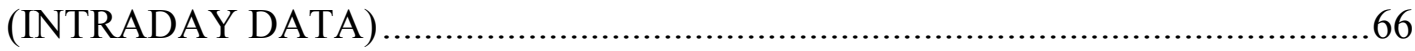

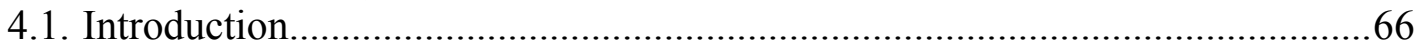

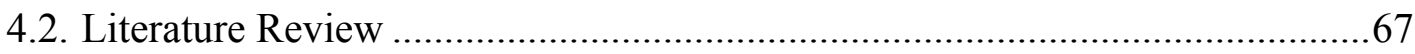

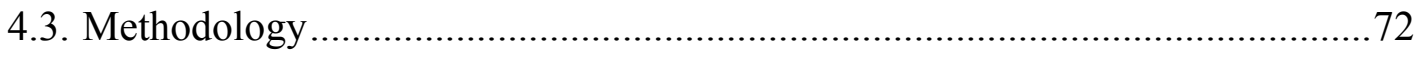

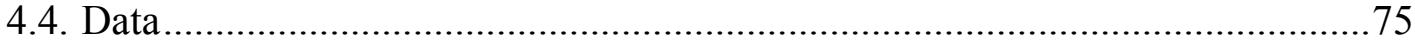


4.5. Empirical Results.

.76

4.6. Summary and Conclusions ........................................................................... 81

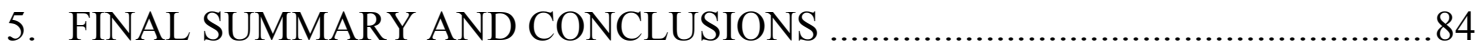

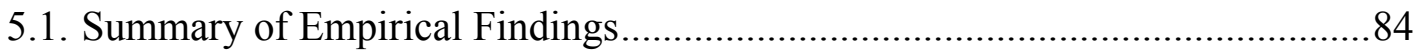

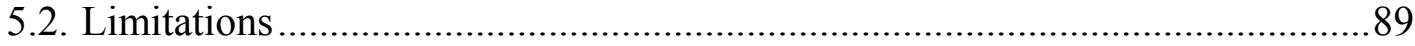

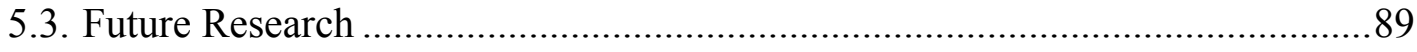

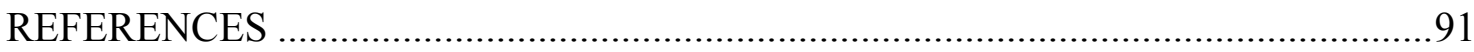

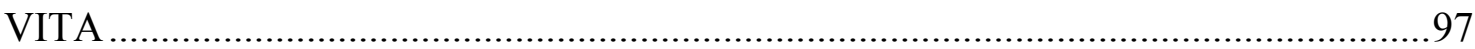




\section{LIST OF TABLES}

TABLE

PAGE

1. Summary Statistics of Spot and 90-Day Forward Rates

2. Tests of Equality of Means Between the Non-Crisis and Crisis Periods .14

3. Day-of-the-Week Effect Over the Entire Sample Period ......................................... 15

4. Day-of-the-Week Effect Over the Non-Crisis Sample Period .................................. 19

5. Day-of-the-Week Effect Over the Crisis Sample Period ........................................23

6. End-of-the-Month Effect Over the Entire Sample Period .....................................27

7. Univariate Tests of Forward Rate as an Unbiased Predictor of Future Spot Rate......52

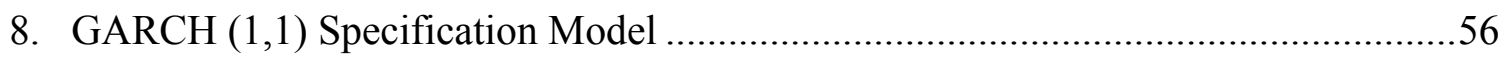

9. Tests of Equality of Estimated Intercepts and Coefficients Between the Non-Crisis and Crisis Periods of the GARCH $(1,1)$ Model ...................................................58

10. Stationarity Tests of Spot and Forward Exchange Rates.....................................59

11. Bivariate Cointegration Rank Tests for Unbiased Forward Rate Hypothesis

12. Error Correction Model for Spot Exchange Rate and Currency Exchange Traded

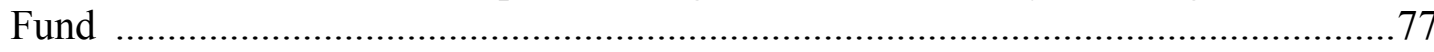

13. Information Share of Spot Exchange Rates and Currency ETFs ............................ 80

14. Test of Equality of Information Share Between the Non-Crisis and Crisis Periods...82 


\section{CHAPTER 1: INTRODUCTION}

\subsection{Motivation and Purpose}

The financial crisis of 2007 has created one of the greatest financial distresses since the Great Depression of the 1930s. This crisis differs from former crises (Asian Crisis in 1997 and Russian Crisis in 1998) because it is both severe and global. The basic underlying causes began with the failure resolution in the collapse of the equity markets at the end of the 1990s. Shortly thereafter, the techno bubble of the early 2000 s brought hot money into the real estate market, and under extensive competition in the banking sector, banks loaned vast amounts of money at low interest rates, which finally led to the participation of many unqualified and irresponsible borrowers (subprime debt). By early in the summer of 2007, the fixed income market and banking sector started to run into trouble. The equity market reaction was the second order to fail in later July/August 2008, and the real equity market response (collapse) started in the middle of September 2008 with the bankruptcy of Lehman and the bailout of AIG. From September 15 through late October 2008, nearly all financial markets fell sharply. The global foreign exchange markets were the last ones entering into the financial crisis (see Melvin and Taylor (2009)).

On August 16, 2007, investors in foreign exchange markets lost a lot of money from their carry trade strategies. The failures of Bear Stearns (March 2008) and Lehman (September 2008) as well as the bailout of AIG caused the foreign exchange markets to become more volatile, especially for foreign exchange spread in the fall of 2008, though the Federal Reserve and the U.S. Treasury launched TARP (Troubled Assets Relief Program). Melvin and Taylor (2009) document that in mid-August 2008, the euro began 
to appreciate steadily against the United States dollar (USD), where the U.S. subprime problems and aggressive Federal Reserve interest rate cuts were noticed. Consequently, due to the failure of big financial enterprises in the United States, counterparty and (il)liquidity risks around the world increased dramatically, which finally induced a global financial crisis.

The aim of this dissertation is to investigate the behavior of the foreign exchange market in this latest crisis. The crisis of 2008 was severe, unique, and global and has taken to task many foreign exchange investors. Among the several analyses that are performed, we test the efficiency of the foreign exchange market over different periods, pre-crisis and during-crisis, by using not only daily and high frequency data but also well-known methodologies and new developments. In addition, the behavior of foreign exchange markets is examined and compared over different periods in order to study how investors perform.

\subsection{Background}

Financial market efficiency is one of the central issues in finance. The market is perfectly efficient when asset prices reflect all relevant information. Fama (1970) proposes the theory of efficient markets under the fair game model. When security prices in a market fully reflect all available information, the market is efficient. He divides the overall efficient market hypothesis $(\mathrm{EMH})$ and the empirical tests of the hypothesis into three sub-hypotheses, depending on the information set involved. First, weak form EMH assumes that current stock prices fully reflect all security market information, including historical data such as price, return, and volume. This hypothesis implies that past returns 
(prices) should have no relationship with future returns (prices). The trading rules (technical analysis) cannot benefit information of past returns. Second, semi-strong form EMH asserts that security prices adjust rapidly to all public information. It implies that investors who base their decisions on any important new public information should not earn above the average risk-adjusted profits. Finally, strong form EMH insists that security prices fully reflect all information from public and private sources. It implies that no one can earn higher-than-average risk-adjusted profits.

On the empirical side, the cointegration introduced by Engle and Granger (1987) is one of the most popular methodologies to test weak form foreign exchange market efficiency. Under the weak form efficient market hypothesis, two asset prices cannot be cointegrated because the historical time series of one asset is not able to predict the other asset price. Under the Granger Representation Theorem, two time series are considered to be cointegrated if they are nonstationary. This suggests that there is a long term relationship (equilibrium relationship) between the two time series (exchange rate), and that short term deviations from the long term trend, which are eliminated over time, are useful for prediction, (Engle and Granger, (1987)). On the other hand, when two time series are cointegrated, they present the error-correction representation.

One of the first empirical results of testing foreign exchange market efficiency by applying the Granger approach was suggested by Baillie and Bollerslev (1989). They found cointegration (or unit root) for nominal spot and forward rates for seven exchange rates by applying univariate time series representation. However, the empirical evidence of applying cointegration is substantially inconclusive, which has lead to further econometric model development by Johansen $(1988,1991)$ and Johansen and Juselius 
(1990). Their findings are appealing for further studies; for example, Diebold et al. (1994) address the relation between cointegration and martingale behavior by using Baillie and Bollerslev (1989) data set and then applying Johansen (1988, 1991). Their results reject the cointegration assumption.

\subsection{Prior Literature on the Efficiency of the Foreign Exchange Markets}

The efficiency tests of the foreign exchange market, largely based on daily data, have led to inconclusive results. The varying results arise from differences in the studies time periods, databases, methodologies, and lag lengths. This brief review of the literature can be broken into two strands. The first strand presents pioneering and some theoretical works, and the second strand involves the application of cointegration for testing market efficiency.

The primary studies by MacDonald and Taylor (1989) and Hakkio and Rush (1989) reject the assumption of cointegration at the 5\% significance level by using the Dickey-Fuller test and Engle and Granger representation. More specifically, MacDonald and Taylor (1989) find cointegration for the French franc/USD and Deutsche mark/USD, while Baillie and Bollerslev (1989) assert the cointegration of their sample for seven currencies by using daily nominal spot and forward rates from March 1980 to January 1985 for Johansen's (1988) maximum likelihood technique. Sephton and Larsen (1991) suggest that the level of market efficiency is heavily sensitive to the sample period chosen; this is later confirmed by Barkoulas and Baum (1997). Lajauine and Naka (1992), employing Johansen's (1991) methodology, investigate four currencies traded in Tokyo's foreign exchange market and find evidence against Baillie and Bollerslev 
(1989). The difference is a result of superior methodology and a different data set. This evidence is reconfirmed by Lajauine et al. (1996), whose study shows the efficiency among Tokyo, London, and New York foreign exchange markets.

Diebold et al. (1994) argue the results of Baillie and Bollerslev (1989) by including drift to Johansen's (1991) technique; the results contradict previous findings. The forecasting performance of cointegrated VARs does not operate well, indicating that the null hypothesis of no cointegration cannot be rejected. At the same time, Baillie and Bollerslev (1994) claim that the market is able to be cointegrated because exchange rates are linked through a long memory $\mathrm{I}(d)$ process in which " $d$ " represents between 0 and 1 (called fractional integration).

Dwyer and Wallace (1992) apply cointegration under a pegged exchange rate regime. They argue that exchange rates under pegged regimes are cointegrated regardless of market efficiency. The currencies of the European Monetary System members, which comply with the European Rate Mechanism, exemplify this argument.

The second strand represents the application of cointegration in the foreign exchange market. There are two main alternatives to test the efficiency: the test of unbiasedness of forward rate (or futures) as a good (efficient) predictor of future spot rate (or within-country efficiency) and that of cross rate (triangular arbitrage or cross-border efficiency). Starting from the primary work by Hakkio and Rush (1989), the evidence suggests that the spot and forward Deutsche mark/ USD show a unit root pattern. Christodoulakis and Kalyvitis (1997) use a Bayesian model in the Greek foreign exchange market by arguing that the simple efficiency test excludes extraneous depreciation expectation. They find that the Greek spot and forward exchange markets 
are inefficient over the transition period. However, Doukas and Rahman (1986) document efficiency in the currency futures market over the pre- and post-1979 period by investigating the effect of the Fed's policy and discount rate announcements. The event study of discount rate announcements for five foreign currency futures traded on the International Monetary Market (IMM) shows the unanticipation of economic agents. The Fed's policy change event asserts an informationally efficient market. Some previous researchers argue that the risk premium may cause market inefficiency. This is documented by Liu and He (1992) and Hu (1997), who show that the foreign exchange market risk premium is time-varying volatility, causing the forward rate to be a biased predictor of future spot rate. Crowder $(1994,1996)$ explains that the covariancestationary time-varying risk premium is able to predict future spot rate. However, Ligeralde (1997) documents that the market efficiency test depends on the information set, prediction horizon, and covariance matrix estimator. Furthermore, Ligeralde (1997) rejects a time-varying market risk premium.

Due to increasing financial integration in Europe, Woo (1999), Haug et al. (2000), Rangvid and Sorensen (2002), and Aroskar et al. (2004) study currencies under the European Monetary Union. Under a fixed-but-adjustable exchange rate regime, the null hypothesis of no cointegration is largely rejected. This evidence supports early findings by Dwyer and Wallace (1992). Recently, Kühl (2010) tests the cointegration of five major currencies before and after the introduction of the Euro currency. The null hypothesis of no cointegration is not largely rejected before the introduction of the Euro, but after the Euro was introduced, it increases among currency pairs without an arbitrage opportunity. 


\subsection{Objectives}

The purposes of the dissertation can be summarized as follows:

- To examine the behavior of major currencies before and during the current U.S. financial crisis.

- To explore the efficiency of the foreign exchange market before and during the current U.S. financial crisis by applying several well known methodologies.

- $\quad$ To explore both daily and intra-day data for efficiency testing.

As the current crisis is severe, unique, and global and more importantly, has lasted longer than expected, we provide new evidence on the behavior of investors in foreign exchange markets. Rather than examining only in daily data, we explore the market at the intraday level. The currencies in our study include the Australian dollar (AUD), Canadian dollar (CAD), Swiss franc (CHF), Euro (EUR), British pound (GBP), and Japanese yen (JPY) over the period of 2004-2011. All are very actively traded and important for global financial stability. The currencies are quoted against the United States dollar (USD). 


\section{CHAPTER 2: FUNDAMENTAL ASPECTS OF THE BEHAVIOR OF THE FOREX}

\section{MARKET BEFORE AND DURING THE CRISIS}

The purpose of this chapter is to provide the basic but notable characteristics of daily spot and 90-day forward rates of the Australian dollar (AUD), Canadian dollar (CAD), Swiss franc (CHF), Euro (EUR), British pound (GBP), and Japanese yen (JPY). These currencies are quoted against the United States dollar (USD). We first analyze and present the evidence over the entire sample. Then the non-crisis and crisis periods are covered and the results are compared.

\subsection{Data Description and the Determination of the Periods}

The bid and ask prices are collected from DataStream, which excludes observations on weekends. The entire data period is from January 2004 to February 2011. In order to identify the pre-recession or non-crisis period, we follow the announcement by the Business Cycle Dating Committee, National Bureau of Economic Research (NBER), which states the U.S. economy entered a recession in December 2007 (see Labonte (2009)), although stock prices evidently started declining in the fall of 2008 . For the purpose of our analyses, the non-crisis period is from 2004 to 2007, totaling four years. The remaining interval is defined as the crisis period. There are 1,868 observations

over the entire sample period, of which 1,043 observations are from the non-crisis period, and 825 observations are noted during the crisis period. 


\subsection{Summary Statistics}

Table 1 presents the basic statistics of the spot and 90-day forward rates of the Australian dollar (AUD), Canadian dollar (CAD), Swiss franc (CHF), Euro (EUR), British pound (GBP), and Japanese yen (JPY) over different time periods. The spot and forward rates are the mid-point of their corresponding bid and ask prices. Panels A, B, and $\mathrm{C}$ show the results over the entire sample, non-crisis, and crisis periods, respectively. In general, from Panel A, the first four moments of spot and forward rates behave similarly for all currencies. For example, the spot and forward rates of the AUD, CAD, and GBP are right skewed, but those of the CHF, EUR, and JPY are left skewed. All spot and forward exchange rates are platykurtic. The more striking result is that the standard deviations of the spot and 90-day forward rates for the entire sample are not significantly different.

Panel B of Table 1 presents the results over the non-crisis period. The difference in mean values of spot and its corresponding forward rates of all currencies is statistically different. Compared with the results of the entire sample as shown in Panel A of Table 1, the average spot and forward rates are strengthened, with the exception of the GBP and EUR. This implies that investors positively view and trust the economy in the United States. The negative skews are noticeable for all spot rates, which is inconsistent with the forward rate. The CAD and CHF forward rates are positively skewed. Notably, the standard deviations of the spot and forward rates are not statistically different. Somewhat expected, the standard deviations over the non-crisis period are smaller than those of the entire sample period. 
Panel $\mathrm{C}$ of Table 1 presents the results throughout the crisis period. The USD is weakened against other currencies, with the exception of the GBP. The difference in mean values of the spot and its corresponding forward rates are not statisticvally different, which contrasts with the results during the non-crisis period. The standard deviations of the AUD, GBP, and JPY are tremendously greater than in the non-crisis period; however, those of the CHF and EUR are minimally larger. The most interesting result, and somewhat unexpected, is that the standard deviation of the CAD in the crisis period is less than in the non-crisis period. A possible explanation is the regional effect. This is evident by the strong positive correlation between the CAD and USD. However, the properties of higher moments differ from those of previous results. The forward rates are positively skewed, with the exception of the GBP. The AUD and CAD are leptokurtic, while the other currencies are platykurtic. From these preliminary results, the behavior of the GBP seems to perform differently from the other currencies.

In Table 1, we also provide the test of normality of the currencies. The SharpioWilks normality statistical test is far beyond the critical value irrespective of the time period, which suggests that the spot and 90-day forward rates of all currencies are far from a normal distribution. 
Table 1 Summary Statistics of Spot and 90-Day Forward Rates

This table presents the basic statistics of the spot and 90-day forward rates of the Australian dollar (AUD), Canadian dollar (CAD), Swiss franc (CHF), Euro (EUR), British pound (GBP), and Japanese yen (JPY) over different time periods, respectively. The non-crisis period begins January 2004 and ends December 2007. The crisis period begins January 2008 and ends February 2011. The spot rate is the mid-point of bid and ask prices. The currencies are quoted against United States dollar (USD). There are 1,868 observations over the entire sample period, which consists of 1,043 observations over the non-crisis period and 825 observation over the crisis period. The Sharpio-Wilk normality tests are presented and the $p$-value numbers are shown in brackets. $* * *$ is significant at the $1 \%$ level.

Panel A: Full Sample Period: January 2004-February 2011

\begin{tabular}{|c|c|c|c|c|c|c|c|c|c|c|c|c|}
\hline \multirow[t]{2}{*}{ Currency } & \multicolumn{6}{|c|}{ Spot Rate } & \multicolumn{6}{|c|}{ 90-Day Forward Rate } \\
\hline & Mean & Median & $\begin{array}{l}\text { Std. } \\
\text { Dev. }\end{array}$ & Skewness & Kurtosis & $\begin{array}{l}\text { Sharpio- } \\
\text { Wilk }\end{array}$ & Mean & Median & $\begin{array}{l}\text { Std. } \\
\text { Dev. }\end{array}$ & Skewness & Kurtosis & $\begin{array}{l}\text { Sharpio- } \\
\text { Wilk }\end{array}$ \\
\hline AUD & 1.2459 & 1.2748 & 0.1365 & 0.0446 & -0.6645 & $\begin{array}{c}0.9747 * * * \\
{[0.000]}\end{array}$ & 1.2684 & 1.2936 & 0.1305 & 0.0658 & -0.6745 & $\begin{array}{c}0.9749 * * * \\
{[0.000]}\end{array}$ \\
\hline CAD & 1.1336 & 1.1279 & 0.1059 & 0.3739 & -0.8245 & $\begin{array}{c}0.9584 * * * \\
{[0.000]}\end{array}$ & 1.1445 & 1.1386 & 0.1083 & 0.3312 & -0.9097 & $\begin{array}{c}0.9599 * * * \\
{[0.000]}\end{array}$ \\
\hline $\mathrm{CHF}$ & 1.1600 & 1.1814 & 0.1010 & -0.3596 & -1.0367 & $\begin{array}{c}0.9486 * * * \\
{[0.000]}\end{array}$ & 1.1670 & 1.1875 & 0.0942 & -0.3151 & -1.0200 & $\begin{array}{c}0.9566^{* * *} * \\
{[0.000]}\end{array}$ \\
\hline EUR & 0.7560 & 0.7618 & 0.0555 & -0.3845 & -0.6455 & $\begin{array}{c}0.9695 * * * \\
{[0.000]}\end{array}$ & 0.7586 & 0.7662 & 0.0568 & -0.3455 & -0.7215 & $\begin{array}{c}0.9717 * * * \\
{[0.000]}\end{array}$ \\
\hline GBP & 0.5689 & 0.5522 & 0.0596 & 0.6150 & -0.7149 & $\begin{array}{c}0.9302 * * * \\
{[0.000]}\end{array}$ & 0.5687 & 0.5549 & 0.0579 & 0.6760 & -0.5156 & $\begin{array}{c}0.9343 * * * \\
{[0.000]}\end{array}$ \\
\hline JPY & 104.80 & 107.10 & 11.496 & -0.4210 & -0.9775 & $\begin{array}{c}0.9417 * * * \\
{[0.000]}\end{array}$ & 105.01 & 107.30 & 10.261 & -0.5049 & -0.7888 & $\begin{array}{c}0.9450 * * * \\
{[0.000]}\end{array}$ \\
\hline
\end{tabular}


Panel B: Non-Crisis Sample Period: January 2004-December 2007

\begin{tabular}{|c|c|c|c|c|c|c|c|c|c|c|c|c|}
\hline \multirow[t]{2}{*}{ Currency } & \multicolumn{6}{|c|}{ Spot Rate } & \multicolumn{6}{|c|}{ 90-Day Forward Rate } \\
\hline & Mean & Median & $\begin{array}{l}\text { Std. } \\
\text { Dev. }\end{array}$ & Skewness & Kurtosis & $\begin{array}{l}\text { Sharpio- } \\
\text { Wilk }\end{array}$ & Mean & Median & $\begin{array}{l}\text { Std. } \\
\text { Dev. }\end{array}$ & Skewness & Kurtosis & $\begin{array}{l}\text { Sharpio- } \\
\text { Wilk }\end{array}$ \\
\hline AUD & 1.2987 & 1.3093 & 0.077 & -0.6314 & 0.3262 & $\begin{array}{c}0.9533 * * * \\
{[0.000]}\end{array}$ & 1.3231 & 1.3245 & 0.0691 & -0.2233 & 0.2423 & $\begin{array}{c}0.9757 * * * \\
{[0.000]}\end{array}$ \\
\hline CAD & 1.1801 & 1.1718 & 0.0984 & -0.0539 & -0.2957 & $\begin{array}{c}0.9850 * * * \\
{[0.000]}\end{array}$ & 1.2006 & 1.1807 & 0.0909 & 0.2141 & -0.8444 & $\begin{array}{c}0.9679 * * * \\
{[0.000]}\end{array}$ \\
\hline $\mathrm{CHF}$ & 1.2356 & 1.2377 & 0.0469 & -0.3667 & -0.2831 & $\begin{array}{c}0.9824 * * * \\
{[0.000]}\end{array}$ & 1.2378 & 1.2366 & 0.0441 & 0.0015 & -0.3478 & $\begin{array}{c}0.9946 * * * \\
{[0.000]}\end{array}$ \\
\hline EUR & 0.7842 & 0.7857 & 0.0419 & -0.5323 & -0.3345 & $\begin{array}{c}0.9619 * * * \\
{[0.000]}\end{array}$ & 0.7919 & 0.7909 & 0.0369 & -0.1372 & -0.9295 & $\begin{array}{c}0.9750 * * * \\
{[0.000]}\end{array}$ \\
\hline GBP & 0.5348 & 0.5369 & 0.0260 & -0.2074 & -0.9101 & $\begin{array}{c}0.9724 * * * \\
{[0.000]}\end{array}$ & 0.5425 & 0.5439 & 0.0266 & -0.0200 & -0.6687 & $\begin{array}{c}0.9833 * * * \\
{[0.000]}\end{array}$ \\
\hline JPY & 113.10 & 113.93 & 5.3877 & -0.1443 & -1.0548 & $\begin{array}{c}0.9664^{* * *} \\
{[0.000]}\end{array}$ & 111.82 & 111.31 & 5.1034 & -0.0331 & -1.0322 & $\begin{array}{c}0.9712 * * * \\
{[0.000]}\end{array}$ \\
\hline
\end{tabular}

Panel C: Crisis Sample Period: January 2008-February 2011

\begin{tabular}{|c|c|c|c|c|c|c|c|c|c|c|c|c|}
\hline \multirow[t]{2}{*}{ Currency } & \multicolumn{6}{|c|}{ Spot Rate } & \multicolumn{6}{|c|}{ 90-Day Forward Rate } \\
\hline & Mean & Median & $\begin{array}{l}\text { Std. } \\
\text { Dev. }\end{array}$ & Skewness & Kurtosis & $\begin{array}{l}\text { Sharpio- } \\
\text { Wilk }\end{array}$ & Mean & Median & $\begin{array}{l}\text { Std. } \\
\text { Dev. }\end{array}$ & Skewness & Kurtosis & $\begin{array}{l}\text { Sharpio- } \\
\text { Wilk }\end{array}$ \\
\hline AUD & 1.1792 & 1.1174 & 0.1633 & 1.1223 & 0.0983 & $\begin{array}{c}0.8484 * * * \\
{[0.000]}\end{array}$ & 1.1993 & 1.1381 & 0.1549 & 1.2224 & 0.2743 & $\begin{array}{c}0.8242 * * * \\
{[0.000]}\end{array}$ \\
\hline CAD & 1.0748 & 1.0445 & 0.0834 & 1.1491 & 0.0410 & $\begin{array}{c}0.8287 * * * \\
{[0.000]}\end{array}$ & 1.0737 & 1.0449 & 0.0845 & 1.0679 & 0.0079 & $\begin{array}{c}0.8578 * * * \\
{[0.000]}\end{array}$ \\
\hline $\mathrm{CHF}$ & 1.0644 & 1.0607 & 0.0629 & 0.1605 & -0.5238 & $\begin{array}{c}0.9889 * * * \\
{[0.000]}\end{array}$ & 1.0775 & 1.0742 & 0.0573 & 0.1771 & -0.7341 & $\begin{array}{c}0.9834 * * * \\
{[0.000]}\end{array}$ \\
\hline EUR & 0.7203 & 0.7211 & 0.0498 & -0.0383 & -0.8553 & $\begin{array}{c}0.9778 * * * \\
{[0.000]}\end{array}$ & 0.7165 & 0.7110 & 0.0491 & 0.2179 & -0.8372 & $\begin{array}{c}0.9732 * * * \\
{[0.000]}\end{array}$ \\
\hline GBP & 0.6121 & 0.6254 & 0.0620 & -0.5965 & -0.7195 & $\begin{array}{c}0.9037 * * * \\
{[0.000]}\end{array}$ & 0.6018 & 0.6181 & 0.0689 & -0.3567 & -1.1966 & $\begin{array}{c}0.9104 * * * \\
{[0.000]}\end{array}$ \\
\hline JPY & 94.306 & 92.870 & 8.0831 & 0.2965 & -0.9017 & $\begin{array}{c}0.9542 * * * \\
{[0.000]}\end{array}$ & 96.407 & 94.545 & 8.5318 & 0.3499 & -0.7893 & $\begin{array}{c}0.9630 * * * \\
{[0.000]}\end{array}$ \\
\hline
\end{tabular}




\subsection{Tests of Equality of Means Between the Non-Crisis and Crisis Periods}

We further analyze by comparing the equality of the means between the non-crisis and crisis periods in order to examine how investors view the direction of exchange rates as shown in Table 2. Both parametric and non-parametric statistic tests are utilized. The paired t-test (parametric test) is used to test the equality of means by assuming the equality of variance over the two different periods. The assumption of variance equality is supported by some currencies as shown in Table 1, which shows that there is a slight difference in the standard deviations between spot and forward rates. The Wilcoxon signed test is employed for the nonparametric approach, which does not require the assumption of distribution. The null and alternative hypotheses are as follows:

$$
\begin{aligned}
& \mathrm{H}_{0}: \mu_{i, j}^{\text {Non-Crisis }}=\mu_{i, j}^{\text {Crisis }} \\
& \mathrm{H}_{1}: \mu_{i, j}^{\text {Non-Crisis }} \neq \mu_{i, j}^{\text {Crisis }}
\end{aligned}
$$

where $\mu$ is the mean of currency $i$ and $j$ is the spot and forward exchange rates, respectively. Non-Crisis and Crisis denote non-crisis and crisis periods, respectively.

Both the paired t-test and Wilcoxon signed test reject the null hypothesis of the equality of means between the two periods at the $1 \%$ significance level. Thus, we can conclude that the difference in means of the non-crisis and crisis periods is economically and statistically significant. It is also intriguing to note that the Wilcoxon signed test does not reject the equality of the mean of GBP forward rate. 
Table 2 Tests of Equality of Means Between the Non-crisis and Crisis Periods

This table presents the test of equality of means between the before and crisis periods. Both parametric (paired t-test) and nonparametric (Wilcoxon signed test) are used for the analysis. *** is the significant at the $1 \%$ level.

\begin{tabular}{ccccc}
\hline \multirow{2}{*}{ Currency } & \multicolumn{2}{c}{ Spot Rate } & \multicolumn{2}{c}{$90-$ Day Forward Rate } \\
\cline { 2 - 5 } & Paired T-Test & Wilcoxon Signed Test & Paired T-Test & Wilcoxon Signed Test \\
\hline AUD & $24.02^{* * *}$ & $224366^{* * *}$ & $23.71^{* * *}$ & $223067^{* * *}$ \\
CAD & $35.45^{* * *}$ & $264216^{* * *}$ & $34.44^{* * *}$ & $264078^{* * *}$ \\
CHF & $57.21^{* * *}$ & $270036^{* * *}$ & $55.78^{* * *}$ & $269714^{* * *}$ \\
EUR & $32.11^{* * *}$ & $250154^{* * *}$ & $35.23 * * *$ & $252892 * * *$ \\
GBP & $-27.74 * * *$ & $-37346^{* * *}$ & $-17.44 * * *$ & -1377 \\
JPY & $42.21 * * *$ & $270153^{* * *}$ & $35.05^{* * *}$ & $260905^{* * *}$ \\
\hline
\end{tabular}

\subsection{Day-of-the-Week Effect}

The foreign exchange market is a 24-hour global trading market and is usually inactive during weekends and national holidays. Our database from DataStream excludes data on holidays and weekends. In this section, we examine the behavior of the foreign exchange rates on each day of the week (short seasonality). The quoted spread is computed as an ask price subtracting its corresponding bid price, and the percentage quoted spread is computed as $\frac{\text { Quoted Spread }}{\text { Mid Point }} X 100$. The average realized volatility on each day is presented by the return squared.

Tables 3, 4, and 5 present the basic statistics and spread behaviors for the entire, non-crisis, and crisis periods, respectively. For the full sample period, the standard deviations of both spot and forward rates are generally largest on Mondays and Fridays with the exception of the CAD and EUR. The forward markets are less volatile than their corresponding spot markets, but this is not true for the CAD. The standard deviations of the CAD and EUR are relatively stable over the entire week, although they seem to be largest on Wednesdays. In general, basic statistics of spot and forward markets are quite 
similar for all currencies. It is interesting to note that all currencies possess negative excess kurtosis, while the third-moment characteristics are varied: positive for the AUD, $\mathrm{CAD}$, and GBP, and negative for the rest. In addition, the forward markets have wider spreads than their corresponding spot markets, which is consistent with less activity and market participants in forward markets. The quoted spread and percentage quoted spread are relatively stable over a week, with the exception of the AUD. The percentage quoted spreads of the AUD are varied, with widest spreads happening on Tuesdays.

Table 3 Day-of-the-Week Effect Over the Entire Sample Period

This table presents the summary statistics on each day of the Australian dollar (AUD), Canadian dollar (CAD), Swiss franc (CHF), Euro (EUR), British pound (GBP), and Japanese yen (JPY) respectively over the entire sample period. The quoted spread is defined as the difference between quoted ask and quoted bid prices. \% quoted spread is equal to $\frac{\text { Quoted Spread }}{\text { Mid Point }} X 100$. The $\mathrm{r}^{2}$ is a proxy of daily realized volatility measure.

Panel A: AUD

\begin{tabular}{|c|c|c|c|c|c|c|c|}
\hline \multirow[t]{2}{*}{ Day } & \multicolumn{7}{|c|}{ Spot Rate } \\
\hline & Mean & Std. Dev. & Skew & Kurtosis & $\begin{array}{l}\text { Quoted } \\
\text { Spread }\end{array}$ & $\begin{array}{c}\% \text { Quoted } \\
\text { Spread }\end{array}$ & $\mathrm{r}^{2} * 10^{4}$ \\
\hline Monday & 1.2457 & 0.1371 & 0.0427 & -0.6280 & 0.0008 & 0.0621 & 4.8568 \\
\hline Tuesday & 1.2461 & 0.1360 & 0.0496 & -0.6635 & 0.0008 & 0.0627 & 4.1820 \\
\hline Wednesday & 1.2461 & 0.1363 & 0.0250 & -0.7252 & 0.0008 & 0.0612 & 4.2364 \\
\hline Thursday & 1.2457 & 0.1360 & 0.0341 & -0.6738 & 0.0008 & 0.0624 & 3.9814 \\
\hline Friday & 1.2462 & 0.1368 & 0.0721 & -0.6054 & 0.0008 & 0.0622 & 4.5329 \\
\hline \multirow[t]{2}{*}{ Day } & \multicolumn{7}{|c|}{ 90-Day Forward Rate } \\
\hline & Mean & Std. Dev. & Skew & Kurtosis & $\begin{array}{l}\text { Quoted } \\
\text { Spread }\end{array}$ & $\begin{array}{c}\% \text { Quoted } \\
\text { Spread } \\
\end{array}$ & $\mathrm{r}^{2} * 10^{4}$ \\
\hline Monday & 1.2541 & 0.1361 & 0.0785 & -0.6075 & 0.0013 & 0.1021 & 4.7802 \\
\hline Tuesday & 1.2544 & 0.1350 & 0.0881 & -0.6389 & 0.0013 & 0.1022 & 4.1450 \\
\hline Wednesday & 1.2544 & 0.1353 & 0.0635 & -0.7012 & 0.0013 & 0.1019 & 4.1969 \\
\hline Thursday & 1.2541 & 0.1351 & 0.0740 & -0.6484 & 0.0013 & 0.1025 & 3.9341 \\
\hline Friday & 1.2547 & 0.1360 & 0.1077 & -0.5894 & 0.0013 & 0.1039 & 4.6358 \\
\hline
\end{tabular}


Panel B: CAD

\begin{tabular}{|c|c|c|c|c|c|c|c|}
\hline \multirow[t]{2}{*}{ Day } & \multicolumn{7}{|c|}{ Spot Rate } \\
\hline & Mean & Std. Dev. & Skew & Kurtosis & $\begin{array}{l}\text { Quoted } \\
\text { Spread }\end{array}$ & $\begin{array}{c}\text { \% Quoted } \\
\text { Spread }\end{array}$ & $\mathrm{r}^{2} * 10^{4}$ \\
\hline Monday & 1.1334 & 0.1058 & 0.3779 & -0.8278 & 0.0006 & 0.0533 & 2.2103 \\
\hline Tuesday & 1.1337 & 0.1055 & 0.3783 & -0.8215 & 0.0006 & 0.0533 & 2.6526 \\
\hline Wednesday & 1.1335 & 0.1062 & 0.3804 & -0.8064 & 0.0006 & 0.0533 & 2.4198 \\
\hline Thursday & 1.1338 & 0.1058 & 0.3707 & -0.8229 & 0.0006 & 0.0532 & 2.4037 \\
\hline Friday & 1.1336 & 0.1059 & 0.3684 & -0.8236 & 0.0006 & 0.0533 & 2.3700 \\
\hline \multirow[t]{2}{*}{ Day } & \multicolumn{7}{|c|}{ 90-Day Forward Rate } \\
\hline & Mean & Std. Dev. & Skew & Kurtosis & $\begin{array}{l}\text { Quoted } \\
\text { Spread }\end{array}$ & $\begin{array}{c}\text { \% Quoted } \\
\text { Spread }\end{array}$ & $\mathrm{r}^{2} * 10^{4}$ \\
\hline Monday & 1.1330 & 0.1059 & 0.4212 & -0.7705 & 0.0012 & 0.1061 & 2.1921 \\
\hline Tuesday & 1.1333 & 0.1056 & 0.4216 & -0.7647 & 0.0012 & 0.1055 & 2.6231 \\
\hline Wednesday & 1.1330 & 0.1063 & 0.4239 & -0.7493 & 0.0012 & 0.1057 & 2.3912 \\
\hline Thursday & 1.1334 & 0.1060 & 0.4154 & -0.7646 & 0.0012 & 0.1057 & 2.3874 \\
\hline Friday & 1.1332 & 0.1060 & 0.4120 & -0.7660 & 0.0012 & 0.1056 & 2.3448 \\
\hline
\end{tabular}

Panel C: CHF

\begin{tabular}{|c|c|c|c|c|c|c|c|}
\hline \multirow[t]{2}{*}{ Day } & \multicolumn{7}{|c|}{ Spot Rate } \\
\hline & Mean & Std. Dev. & Skew & Kurtosis & $\begin{array}{l}\text { Quoted } \\
\text { Spread }\end{array}$ & $\begin{array}{c}\text { \% Quoted } \\
\text { Spread }\end{array}$ & $\mathrm{r}^{2} * 10^{4}$ \\
\hline Monday & 1.1599 & 0.1015 & -0.3661 & -1.0247 & 0.0006 & 0.0521 & 2.2968 \\
\hline Tuesday & 1.1602 & 0.1006 & -0.3569 & -1.0370 & 0.0006 & 0.0520 & 2.1683 \\
\hline Wednesday & 1.1599 & 0.1010 & -0.3600 & -1.0402 & 0.0006 & 0.0520 & 2.1543 \\
\hline Thursday & 1.1597 & 0.1007 & -0.3685 & -1.0372 & 0.0006 & 0.0521 & 2.1007 \\
\hline Friday & 1.1601 & 0.1012 & -0.3524 & -1.0366 & 0.0006 & 0.0521 & 2.1654 \\
\hline \multirow[t]{2}{*}{ Day } & \multicolumn{7}{|c|}{ 90-Day Forward Rate } \\
\hline & Mean & Std. Dev. & Skew & Kurtosis & $\begin{array}{l}\text { Quoted } \\
\text { Spread }\end{array}$ & $\begin{array}{c}\text { \% Quoted } \\
\text { Spread }\end{array}$ & $\mathrm{r}^{2} * 10^{4}$ \\
\hline Monday & 1.1548 & 0.0983 & -0.3729 & -0.9904 & 0.0016 & 0.1411 & 2.2948 \\
\hline Tuesday & 1.1551 & 0.0975 & -0.3630 & -1.0028 & 0.0016 & 0.1404 & 2.1534 \\
\hline Wednesday & 1.1548 & 0.0978 & -0.3650 & -1.0055 & 0.0016 & 0.1406 & 2.1530 \\
\hline Thursday & 1.1546 & 0.0975 & -0.3745 & -1.0033 & 0.0016 & 0.1410 & 2.1090 \\
\hline Friday & 1.1551 & 0.0981 & -0.3567 & -1.0035 & 0.0016 & 0.1409 & 2.1618 \\
\hline
\end{tabular}


Panel D: EUR

\begin{tabular}{|c|c|c|c|c|c|c|c|}
\hline \multirow[t]{2}{*}{ Day } & \multicolumn{7}{|c|}{ Spot Rate } \\
\hline & Mean & Std. Dev. & Skew & Kurtosis & $\begin{array}{l}\text { Quoted } \\
\text { Spread }\end{array}$ & $\begin{array}{c}\text { \% Quoted } \\
\text { Spread }\end{array}$ & $r^{2} * 10^{4}$ \\
\hline Monday & 0.7560 & 0.0555 & -0.3897 & -0.6205 & 0.0002 & 0.0304 & 2.2511 \\
\hline Tuesday & 0.7561 & 0.0555 & -0.3827 & -0.6375 & 0.0002 & 0.0303 & 2.1335 \\
\hline Wednesday & 0.7559 & 0.0557 & -0.3895 & -0.6441 & 0.0002 & 0.0302 & 2.1666 \\
\hline Thursday & 0.7558 & 0.0554 & -0.3901 & -0.6581 & 0.0002 & 0.0300 & 1.9992 \\
\hline Friday & 0.7560 & 0.0555 & -0.3769 & -0.6377 & 0.0002 & 0.0303 & 2.0285 \\
\hline \multirow[t]{2}{*}{ Day } & \multicolumn{7}{|c|}{ 90-Day Forward Rate } \\
\hline & Mean & Std. Dev. & Skew & Kurtosis & $\begin{array}{l}\text { Quoted } \\
\text { Spread }\end{array}$ & $\begin{array}{c}\% \text { Quoted } \\
\text { Spread } \\
\end{array}$ & $\mathrm{r}^{2} * 10^{4}$ \\
\hline Monday & 0.7553 & 0.0544 & -0.3678 & -0.6579 & 0.0004 & 0.0514 & 2.2384 \\
\hline Tuesday & 0.7554 & 0.0544 & -0.3609 & -0.6754 & 0.0004 & 0.0509 & 2.1247 \\
\hline Wednesday & 0.7551 & 0.0546 & -0.3648 & -0.6823 & 0.0004 & 0.0510 & 2.1669 \\
\hline Thursday & 0.7551 & 0.0543 & -0.3676 & -0.6923 & 0.0004 & 0.0506 & 2.0037 \\
\hline Friday & 0.7553 & 0.0544 & -0.3539 & -0.6750 & 0.0004 & 0.0508 & 2.0194 \\
\hline
\end{tabular}

Panel E: GBP

\begin{tabular}{|c|c|c|c|c|c|c|c|}
\hline \multirow[t]{2}{*}{ Day } & \multicolumn{7}{|c|}{ Spot Rate } \\
\hline & Mean & Std. Dev. & Skew & Kurtosis & $\begin{array}{l}\text { Quoted } \\
\text { Spread }\end{array}$ & $\begin{array}{c}\text { \% Quoted } \\
\text { Spread }\end{array}$ & $\mathrm{r}^{2} * 10^{4}$ \\
\hline Monday & 0.5692 & 0.0596 & 0.6152 & -0.7224 & 0.0002 & 0.0269 & 2.2850 \\
\hline Tuesday & 0.5689 & 0.0597 & 0.6222 & -0.7060 & 0.0002 & 0.0269 & 2.1823 \\
\hline Wednesday & 0.5689 & 0.0595 & 0.6275 & -0.6582 & 0.0002 & 0.0269 & 2.3674 \\
\hline Thursday & 0.5688 & 0.0594 & 0.6104 & -0.7281 & 0.0002 & 0.0270 & 2.1941 \\
\hline Friday & 0.5690 & 0.0595 & 0.6096 & -0.7334 & 0.0002 & 0.0270 & 2.3689 \\
\hline \multirow[t]{2}{*}{ Day } & \multicolumn{7}{|c|}{ 90-Day Forward Rate } \\
\hline & Mean & Std. Dev. & Skew & Kurtosis & $\begin{array}{l}\text { Quoted } \\
\text { Spread }\end{array}$ & $\begin{array}{c}\text { \% Quoted } \\
\text { Spread }\end{array}$ & $r^{2} * 10^{4}$ \\
\hline Monday & 0.5704 & 0.0590 & 0.6086 & -0.7107 & 0.0003 & 0.0548 & 2.2616 \\
\hline Tuesday & 0.5701 & 0.0591 & 0.6159 & -0.6935 & 0.0003 & 0.0549 & 2.1583 \\
\hline Wednesday & 0.5701 & 0.0589 & 0.6200 & -0.6473 & 0.0003 & 0.0540 & 2.3433 \\
\hline Thursday & 0.5700 & 0.0588 & 0.6043 & -0.7145 & 0.0003 & 0.0548 & 2.1533 \\
\hline Friday & 0.5702 & 0.0590 & 0.6025 & -0.7214 & 0.0003 & 0.0547 & 2.3403 \\
\hline
\end{tabular}


Panel F: JPY

\begin{tabular}{|c|c|c|c|c|c|c|c|}
\hline \multirow[t]{2}{*}{ Day } & \multicolumn{7}{|c|}{ Spot Rate } \\
\hline & Mean & Std. Dev. & Skew & Kurtosis & $\begin{array}{l}\text { Quoted } \\
\text { Spread }\end{array}$ & $\begin{array}{c}\text { \% Quoted } \\
\text { Spread }\end{array}$ & $\mathrm{r}^{2} * 10^{4}$ \\
\hline Monday & 104.7653 & 11.5114 & -0.4136 & -0.9690 & 0.0599 & 0.0579 & 2.4270 \\
\hline Tuesday & 104.8130 & 11.4844 & -0.4330 & -0.9633 & 0.0599 & 0.0580 & 1.8854 \\
\hline Wednesday & 104.8163 & 11.4911 & -0.4234 & -0.9968 & 0.0598 & 0.0578 & 2.0147 \\
\hline Thursday & 104.8051 & 11.4701 & -0.4272 & -0.9688 & 0.0600 & 0.0580 & 1.9127 \\
\hline Friday & 104.7864 & 11.5056 & -0.4148 & -0.9776 & 0.0599 & 0.0579 & 2.3413 \\
\hline \multirow[t]{2}{*}{ Day } & \multicolumn{7}{|c|}{ 90-Day Forward Rate } \\
\hline & Mean & Std. Dev. & Skew & Kurtosis & $\begin{array}{l}\text { Quoted } \\
\text { Spread }\end{array}$ & $\begin{array}{c}\text { \% Quoted } \\
\text { Spread }\end{array}$ & $\mathrm{r}^{2} * 10^{4}$ \\
\hline Monday & 104.0791 & 11.0397 & -0.4449 & -0.9307 & 0.1290 & 0.1245 & 2.4264 \\
\hline Tuesday & 104.1238 & 11.0116 & -0.4646 & -0.9242 & 0.1286 & 0.1240 & 1.8764 \\
\hline Wednesday & 104.1199 & 11.0117 & -0.4548 & -0.9602 & 0.1285 & 0.1239 & 2.0263 \\
\hline Thursday & 104.1141 & 10.9973 & -0.4583 & -0.9317 & 0.1289 & 0.1244 & 1.9188 \\
\hline Friday & 104.0987 & 11.0340 & -0.4457 & -0.9408 & 0.1288 & 0.1243 & 2.3497 \\
\hline
\end{tabular}

In this section, we further compare and analyze the day-of-the-week effect over the non-crisis and crisis periods (as presented in Tables 4 and 5, respectively). The USD is weakened during the crisis period due to subprime problems, but strengthened against the GBP. However, the difference of dispersions of the means on each day is diminished for both periods. The standard deviations of forward markets are slightly lower than those of spot markets over the crisis period, but slightly higher over the non-crisis period. Friday is still the most volatile day for all currencies in both periods. Interestingly, the skewness of the AUD and CAD is positive during the non-crisis period, and the excess kurtosis of the AUD is positive, which contradicts the results from the full sample and crisis periods (as shown in Tables 3 and 5). Moreover, higher moments of spot and forward markets over the non-crisis and crisis periods are significantly different for some currencies. For example, the spot and forward AUD over the non-crisis period is left skewed, but right skewed over the crisis period. In conclusion, the behaviors of higher moments in both spot and forward markets are significantly economically different. 
However, the behaviors of quoted spread and percentage quoted spread over the non-crisis and crisis periods are relatively similar to those of the full sample period. The quoted spread and percentage quoted spread are stable over a week. However, the largest quoted spread and percentage quoted spread for the AUD are on Tuesdays. In sum, the spreads in forward markets are still larger than in spot markets in both time periods.

\section{Table 4 Day-of-the-Week Effect Over the Non-Crisis Sample Period}

This table presents the summary statistics on each day of the Australian dollar (AUD), Canadian dollar (CAD), Swiss franc (CHF), Euro (EUR), British pound (GBP), and Japanese yen (JPY) respectively over the non-crisis sample period. The quoted spread is defined as the difference between quoted ask and quoted bid prices. \% quoted spread is equal to $\frac{\text { Quoted Spread }}{\text { Mid Point }} X 100$. The $r^{2}$ is a proxy of daily realized volatility measure.

Panel A: AUD

\begin{tabular}{|c|c|c|c|c|c|c|c|}
\hline \multirow[t]{2}{*}{ Day } & \multicolumn{7}{|c|}{ Spot Rate } \\
\hline & Mean & Std. Dev. & Skew & Kurtosis & $\begin{array}{l}\text { Quoted } \\
\text { Spread }\end{array}$ & $\begin{array}{c}\% \text { Quoted } \\
\text { Spread }\end{array}$ & $\mathrm{r}^{2} * 10^{4}$ \\
\hline Monday & 1.2987 & 0.0769 & -0.6255 & 0.3094 & 0.0008 & 0.0644 & 2.4034 \\
\hline Tuesday & 1.2988 & 0.0766 & -0.6157 & 0.3164 & 0.0009 & 0.0657 & 2.3280 \\
\hline Wednesday & 1.2989 & 0.0779 & -0.6439 & 0.3991 & 0.0008 & 0.0642 & 2.3060 \\
\hline Thursday & 1.2987 & 0.0773 & -0.6286 & 0.3974 & 0.0008 & 0.0652 & 2.6304 \\
\hline Friday & 1.2985 & 0.0772 & -0.6611 & 0.3517 & 0.0008 & 0.0649 & 2.2082 \\
\hline \multirow[t]{2}{*}{ Day } & \multicolumn{7}{|c|}{ 90-Day Forward Rate } \\
\hline & Mean & Std. Dev. & Skew & Kurtosis & $\begin{array}{l}\text { Quoted } \\
\text { Spread }\end{array}$ & $\begin{array}{c}\% \text { Quoted } \\
\text { Spread }\end{array}$ & $\mathrm{r}^{2} * 10^{4}$ \\
\hline Monday & 1.3052 & 0.0787 & -0.5599 & 0.2890 & 0.0014 & 0.1058 & 2.4016 \\
\hline Tuesday & 1.3053 & 0.0783 & -0.5504 & 0.2989 & 0.0014 & 0.1056 & 2.3231 \\
\hline Wednesday & 1.3055 & 0.0797 & -0.5724 & 0.3655 & 0.0014 & 0.1052 & 2.3019 \\
\hline Thursday & 1.3053 & 0.0791 & -0.5591 & 0.3678 & 0.0014 & 0.1054 & 2.6407 \\
\hline Friday & 1.3053 & 0.0792 & -0.5979 & 0.3056 & 0.0014 & 0.1052 & 2.5608 \\
\hline
\end{tabular}


Panel B: CAD

\begin{tabular}{|c|c|c|c|c|c|c|c|}
\hline \multirow[t]{2}{*}{ Day } & \multicolumn{7}{|c|}{ Spot Rate } \\
\hline & Mean & Std. Dev. & Skew & Kurtosis & $\begin{array}{l}\text { Quoted } \\
\text { Spread }\end{array}$ & $\begin{array}{c}\text { \% Quoted } \\
\text { Spread }\end{array}$ & $\mathrm{r}^{2} * 10^{4}$ \\
\hline Monday & 1.1798 & 0.0985 & -0.0525 & -0.3146 & 0.0006 & 0.0512 & 1.2354 \\
\hline Tuesday & 1.1803 & 0.0976 & -0.0487 & -0.2664 & 0.0006 & 0.0512 & 1.2739 \\
\hline Wednesday & 1.1804 & 0.0986 & -0.0450 & -0.2579 & 0.0006 & 0.0512 & 1.3473 \\
\hline Thursday & 1.1806 & 0.0981 & -0.0471 & -0.2977 & 0.0006 & 0.0512 & 1.3947 \\
\hline Friday & 1.1794 & 0.0988 & -0.0770 & -0.2556 & 0.0006 & 0.0512 & 1.2162 \\
\hline \multirow[t]{2}{*}{ Day } & \multicolumn{7}{|c|}{ 90-Day Forward Rate } \\
\hline & Mean & Std. Dev. & Skew & Kurtosis & $\begin{array}{l}\text { Quoted } \\
\text { Spread }\end{array}$ & $\begin{array}{c}\% \text { Quoted } \\
\text { Spread }\end{array}$ & $\mathrm{r}^{2} * 10^{4}$ \\
\hline Monday & 1.1787 & 0.0999 & 0.0021 & -0.3561 & 0.0012 & 0.1028 & 1.2306 \\
\hline Tuesday & 1.1792 & 0.0991 & 0.0067 & -0.3123 & 0.0012 & 0.1027 & 1.2677 \\
\hline Wednesday & 1.1793 & 0.1000 & 0.0105 & -0.3023 & 0.0012 & 0.1025 & 1.3425 \\
\hline Thursday & 1.1794 & 0.0996 & 0.0091 & -0.3424 & 0.0012 & 0.1026 & 1.3893 \\
\hline Friday & 1.1783 & 0.1002 & -0.0209 & -0.3009 & 0.0012 & 0.1024 & 1.2133 \\
\hline
\end{tabular}

Panel C: CHF

\begin{tabular}{|c|c|c|c|c|c|c|c|}
\hline \multirow[t]{2}{*}{ Day } & \multicolumn{7}{|c|}{ Spot Rate } \\
\hline & Mean & Std. Dev. & Skew & Kurtosis & $\begin{array}{l}\text { Quoted } \\
\text { Spread }\end{array}$ & $\begin{array}{c}\text { \% Quoted } \\
\text { Spread }\end{array}$ & $\mathrm{r}^{2} * 10^{4}$ \\
\hline Monday & 1.2356 & 0.0473 & -0.3566 & -0.3259 & 0.0006 & 0.0486 & 1.6468 \\
\hline Tuesday & 1.2358 & 0.0467 & -0.3580 & -0.2718 & 0.0006 & 0.0486 & 1.4641 \\
\hline Wednesday & 1.2357 & 0.0467 & -0.3789 & -0.2515 & 0.0006 & 0.0485 & 1.4652 \\
\hline Thursday & 1.2352 & 0.0463 & -0.4058 & -0.1820 & 0.0006 & 0.0486 & 1.4475 \\
\hline Friday & 1.2355 & 0.0475 & -0.3477 & -0.2930 & 0.0006 & 0.0486 & 1.6732 \\
\hline \multirow[t]{2}{*}{ Day } & \multicolumn{7}{|c|}{ 90-Day Forward Rate } \\
\hline & Mean & Std. Dev. & Skew & Kurtosis & $\begin{array}{l}\text { Quoted } \\
\text { Spread }\end{array}$ & $\begin{array}{c}\% \text { Quoted } \\
\text { Spread }\end{array}$ & $r^{2} * 10^{4}$ \\
\hline Monday & 1.2276 & 0.0466 & -0.3478 & -0.3845 & 0.0016 & 0.1336 & 1.6497 \\
\hline Tuesday & 1.2278 & 0.0460 & -0.3497 & -0.3222 & 0.0016 & 0.1335 & 1.4615 \\
\hline Wednesday & 1.2276 & 0.0460 & -0.3635 & -0.3120 & 0.0016 & 0.1332 & 1.4624 \\
\hline Thursday & 1.2271 & 0.0456 & -0.3949 & -0.2311 & 0.0016 & 0.1336 & 1.4466 \\
\hline Friday & 1.2276 & 0.0469 & -0.3364 & -0.3614 & 0.0016 & 0.1335 & 1.6746 \\
\hline
\end{tabular}


Panel D: EUR

\begin{tabular}{|c|c|c|c|c|c|c|c|}
\hline \multirow[t]{2}{*}{ Day } & \multicolumn{7}{|c|}{ Spot Rate } \\
\hline & Mean & Std. Dev. & Skew & Kurtosis & $\begin{array}{l}\text { Quoted } \\
\text { Spread }\end{array}$ & $\begin{array}{c}\% \text { Quoted } \\
\text { Spread } \\
\end{array}$ & $r^{2} * 10^{4}$ \\
\hline Monday & 0.7842 & 0.0419 & -0.5273 & -0.3674 & 0.0003 & 0.0319 & 1.3029 \\
\hline Tuesday & 0.7844 & 0.0417 & -0.5244 & -0.3311 & 0.0002 & 0.0314 & 1.1683 \\
\hline Wednesday & 0.7843 & 0.0419 & -0.5340 & -0.3220 & 0.0003 & 0.0318 & 1.2019 \\
\hline Thursday & 0.7841 & 0.0417 & -0.5597 & -0.2557 & 0.0002 & 0.0313 & 1.2565 \\
\hline Friday & 0.7841 & 0.0422 & -0.5317 & -0.3125 & 0.0002 & 0.0316 & 1.3517 \\
\hline \multirow[t]{2}{*}{ Day } & \multicolumn{7}{|c|}{ 90-Day Forward Rate } \\
\hline & Mean & Std. Dev. & Skew & Kurtosis & $\begin{array}{l}\text { Quoted } \\
\text { Spread }\end{array}$ & $\begin{array}{c}\% \text { Quoted } \\
\text { Spread }\end{array}$ & $\mathrm{r}^{2} * 10^{4}$ \\
\hline Monday & 0.7822 & 0.0418 & -0.5082 & -0.4352 & 0.0004 & 0.0553 & 1.2949 \\
\hline Tuesday & 0.7825 & 0.0416 & -0.5064 & -0.3984 & 0.0004 & 0.0555 & 1.1632 \\
\hline Wednesday & 0.7823 & 0.0418 & -0.5124 & -0.3904 & 0.0004 & 0.0552 & 1.1994 \\
\hline Thursday & 0.7821 & 0.0416 & -0.5375 & -0.3263 & 0.0004 & 0.0551 & 1.2522 \\
\hline Friday & 0.7822 & 0.0420 & -0.5113 & -0.3844 & 0.0004 & 0.0552 & 1.3468 \\
\hline
\end{tabular}

Panel E: GBP

\begin{tabular}{|c|c|c|c|c|c|c|c|}
\hline \multirow[t]{2}{*}{ Day } & \multicolumn{7}{|c|}{ Spot Rate } \\
\hline & Mean & Std. Dev. & Skew & Kurtosis & $\begin{array}{l}\text { Quoted } \\
\text { Spread }\end{array}$ & $\begin{array}{c}\text { \% Quoted } \\
\text { Spread }\end{array}$ & $\mathrm{r}^{2} * 10^{4}$ \\
\hline Monday & 0.5349 & 0.0259 & -0.1955 & -0.9311 & 0.0002 & 0.0289 & 1.4090 \\
\hline Tuesday & 0.5347 & 0.0259 & -0.2102 & -0.9107 & 0.0002 & 0.0289 & 1.2930 \\
\hline Wednesday & 0.5348 & 0.0261 & -0.2149 & -0.8884 & 0.0002 & 0.0289 & 1.2431 \\
\hline Thursday & 0.5348 & 0.0258 & -0.2329 & -0.8932 & 0.0002 & 0.0290 & 1.3219 \\
\hline Friday & 0.5348 & 0.0260 & -0.1897 & -0.8996 & 0.0002 & 0.0290 & 1.3987 \\
\hline \multirow[t]{2}{*}{ Day } & \multicolumn{7}{|c|}{ 90-Day Forward Rate } \\
\hline & Mean & Std. Dev. & Skew & Kurtosis & $\begin{array}{l}\text { Quoted } \\
\text { Spread }\end{array}$ & $\begin{array}{c}\% \text { Quoted } \\
\text { Spread }\end{array}$ & $r^{2} * 10^{4}$ \\
\hline Monday & 0.5363 & 0.0262 & -0.2387 & -1.0190 & 0.0003 & 0.0572 & 1.4060 \\
\hline Tuesday & 0.5361 & 0.0262 & -0.2529 & -0.9960 & 0.0003 & 0.0573 & 1.2881 \\
\hline Wednesday & 0.5362 & 0.0264 & -0.2561 & -0.9782 & 0.0003 & 0.0570 & 1.2391 \\
\hline Thursday & 0.5363 & 0.0261 & -0.2720 & -0.9808 & 0.0003 & 0.0574 & 1.3185 \\
\hline Friday & 0.5363 & 0.0263 & -0.2308 & -0.9929 & 0.0003 & 0.0573 & 1.3932 \\
\hline
\end{tabular}


Panel F: JPY

\begin{tabular}{|c|c|c|c|c|c|c|c|}
\hline \multirow[t]{2}{*}{ Day } & \multicolumn{7}{|c|}{ Spot Rate } \\
\hline & Mean & Std. Dev. & Skew & Kurtosis & $\begin{array}{l}\text { Quoted } \\
\text { Spread }\end{array}$ & $\begin{array}{c}\text { \% Quoted } \\
\text { Spread }\end{array}$ & $\mathrm{r}^{2} * 10^{4}$ \\
\hline 113.0824 & 5.4326 & -0.1332 & -1.0477 & 0.0600 & 0.0532 & 1.7415 & 113.0824 \\
\hline 113.0960 & 5.3573 & -0.1436 & -1.0902 & 0.0600 & 0.0532 & 1.3927 & 113.0960 \\
\hline 113.1321 & 5.3568 & -0.1905 & -1.0511 & 0.0599 & 0.0530 & 1.3261 & 113.1321 \\
\hline 113.0837 & 5.3519 & -0.1277 & -1.0668 & 0.0600 & 0.0532 & 1.5161 & 113.0837 \\
\hline 113.0854 & 5.4259 & -0.1317 & -1.0070 & 0.0600 & 0.0532 & 1.7057 & 113.0854 \\
\hline \multirow[t]{2}{*}{ Day } & \multicolumn{7}{|c|}{ 90-Day Forward Rate } \\
\hline & Mean & Std. Dev. & Skew & Kurtosis & $\begin{array}{l}\text { Quoted } \\
\text { Spread }\end{array}$ & $\begin{array}{c}\% \text { Quoted } \\
\text { Spread }\end{array}$ & $r^{2} * 10^{4}$ \\
\hline Monday & 112.0378 & 5.1115 & -0.1317 & -0.9599 & 0.1362 & 0.1217 & 1.7390 \\
\hline Tuesday & 112.0508 & 5.0338 & -0.1431 & -1.0005 & 0.1358 & 0.1213 & 1.3896 \\
\hline Wednesday & 112.0735 & 5.0283 & -0.1939 & -0.9600 & 0.1359 & 0.1214 & 1.3241 \\
\hline Thursday & 112.0386 & 5.0253 & -0.1252 & -0.9768 & 0.1359 & 0.1214 & 1.5109 \\
\hline Friday & 112.0415 & 5.1028 & -0.1306 & -0.9102 & 0.1361 & 0.1216 & 1.7010 \\
\hline
\end{tabular}


Table 5 Day-of-the-Week Effect Over the Crisis Sample Period

This table presents the summary statistics on each day of the Australian dollar (AUD), Canadian dollar (CAD), Swiss franc (CHF), Euro (EUR), British pound (GBP), and Japanese yen (JPY) respectively over the crisis sample period. The quoted spread is defined as the difference between quoted ask and quoted bid prices. \% quoted spread is equal to $\frac{\text { Quoted Spread }}{\text { Mid Point }} X 100$. The $\mathrm{r}^{2}$ is a proxy of daily realized volatility measure.

Panel A: AUD

\begin{tabular}{|c|c|c|c|c|c|c|c|}
\hline \multirow[t]{2}{*}{ Day } & \multicolumn{7}{|c|}{ Spot Rate } \\
\hline & Mean & Std. Dev. & Skew & Kurtosis & $\begin{array}{l}\text { Quoted } \\
\text { Spread }\end{array}$ & $\begin{array}{c}\text { \% Quoted } \\
\text { Spread }\end{array}$ & $\mathrm{r}^{2} * 10^{4}$ \\
\hline Monday & 1.1787 & 0.1644 & 1.1281 & 0.1454 & 0.0007 & 0.0591 & 7.9457 \\
\hline Tuesday & 1.1795 & 0.1627 & 1.1361 & 0.1312 & 0.0007 & 0.0589 & 6.5078 \\
\hline Wednesday & 1.1795 & 0.1624 & 1.1050 & 0.0309 & 0.0007 & 0.0574 & 6.6582 \\
\hline Thursday & 1.1786 & 0.1622 & 1.1273 & 0.1262 & 0.0007 & 0.0590 & 5.6844 \\
\hline Friday & 1.1799 & 0.1644 & 1.1580 & 0.2112 & 0.0007 & 0.0588 & 7.4635 \\
\hline \multirow[t]{2}{*}{ Day } & \multicolumn{7}{|c|}{ 90-Day Forward Rate } \\
\hline & Mean & Std. Dev. & Skew & Kurtosis & $\begin{array}{l}\text { Quoted } \\
\text { Spread }\end{array}$ & $\begin{array}{c}\text { \% Quoted } \\
\text { Spread }\end{array}$ & $\mathrm{r}^{2} * 10^{4}$ \\
\hline Monday & 1.1893 & 0.1631 & 1.1292 & 0.1519 & 0.0012 & 0.0975 & 7.7788 \\
\hline Tuesday & 1.1902 & 0.1615 & 1.1405 & 0.1460 & 0.0012 & 0.0978 & 6.4307 \\
\hline Wednesday & 1.1901 & 0.1611 & 1.1098 & 0.0508 & 0.0012 & 0.0977 & 6.5742 \\
\hline Thursday & 1.1894 & 0.1610 & 1.1321 & 0.1426 & 0.0012 & 0.0988 & 5.5645 \\
\hline Friday & 1.1906 & 0.1632 & 1.1614 & 0.2212 & 0.0012 & 0.1023 & 7.2515 \\
\hline
\end{tabular}

Panel B: CAD

\begin{tabular}{|c|c|c|c|c|c|c|c|}
\hline \multirow[t]{2}{*}{ Day } & \multicolumn{7}{|c|}{ Spot Rate } \\
\hline & Mean & Std. Dev. & Skew & Kurtosis & $\begin{array}{l}\text { Quoted } \\
\text { Spread }\end{array}$ & $\begin{array}{c}\% \text { Quoted } \\
\text { Spread }\end{array}$ & $\mathrm{r}^{2} * 10^{4}$ \\
\hline Monday & 1.0746 & 0.0830 & 1.1533 & 0.0572 & 0.0006 & 0.0560 & 3.4392 \\
\hline Tuesday & 1.0749 & 0.0833 & 1.1781 & 0.1229 & 0.0006 & 0.0560 & 4.3823 \\
\hline Wednesday & 1.0743 & 0.0835 & 1.1613 & 0.0781 & 0.0006 & 0.0559 & 3.7653 \\
\hline Thursday & 1.0745 & 0.0832 & 1.1314 & 0.0113 & 0.0006 & 0.0558 & 3.6757 \\
\hline Friday & 1.0755 & 0.0838 & 1.1631 & 0.0869 & 0.0006 & 0.0560 & 3.8246 \\
\hline \multirow[t]{2}{*}{ Day } & \multicolumn{7}{|c|}{ 90-Day Forward Rate } \\
\hline & Mean & Std. Dev. & Skew & Kurtosis & $\begin{array}{l}\text { Quoted } \\
\text { Spread }\end{array}$ & $\begin{array}{c}\% \text { Quoted } \\
\text { Spread }\end{array}$ & $\mathrm{r}^{2} * 10^{4}$ \\
\hline Monday & 1.0751 & 0.0823 & 1.1617 & 0.0704 & 0.0012 & 0.1102 & 3.4041 \\
\hline Tuesday & 1.0754 & 0.0826 & 1.1852 & 0.1331 & 0.0012 & 0.1089 & 4.3236 \\
\hline Wednesday & 1.0748 & 0.0828 & 1.1711 & 0.0963 & 0.0012 & 0.1096 & 3.7067 \\
\hline Thursday & 1.0750 & 0.0824 & 1.1403 & 0.0302 & 0.0012 & 0.1097 & 3.6456 \\
\hline Friday & 1.0760 & 0.0830 & 1.1725 & 0.1051 & 0.0012 & 0.1096 & 3.7713 \\
\hline
\end{tabular}


Panel C: CHF

\begin{tabular}{|c|c|c|c|c|c|c|c|}
\hline \multirow[t]{2}{*}{ Day } & \multicolumn{7}{|c|}{ Spot Rate } \\
\hline & Mean & Std. Dev. & Skew & Kurtosis & $\begin{array}{l}\text { Quoted } \\
\text { Spread }\end{array}$ & $\begin{array}{c}\text { \% Quoted } \\
\text { Spread }\end{array}$ & $\mathrm{r}^{2} * 10^{4}$ \\
\hline Monday & 1.0640 & 0.0636 & 0.1442 & -0.6092 & 0.0006 & 0.0564 & 3.1162 \\
\hline Tuesday & 1.0650 & 0.0622 & 0.1335 & -0.5864 & 0.0006 & 0.0563 & 3.0519 \\
\hline Wednesday & 1.0644 & 0.0626 & 0.1517 & -0.5279 & 0.0006 & 0.0562 & 3.0188 \\
\hline Thursday & 1.0642 & 0.0626 & 0.1651 & -0.4353 & 0.0006 & 0.0566 & 2.9241 \\
\hline Friday & 1.0646 & 0.0633 & 0.2134 & -0.3800 & 0.0006 & 0.0564 & 2.7858 \\
\hline \multirow[t]{2}{*}{ Day } & \multicolumn{7}{|c|}{ 90-Day Forward Rate } \\
\hline & Mean & Std. Dev. & Skew & Kurtosis & $\begin{array}{l}\text { Quoted } \\
\text { Spread }\end{array}$ & $\begin{array}{c}\text { \% Quoted } \\
\text { Spread }\end{array}$ & $\mathrm{r}^{2} * 10^{4}$ \\
\hline Monday & 1.0626 & 0.0628 & 0.1237 & -0.6115 & 0.0016 & 0.1505 & 3.1080 \\
\hline Tuesday & 1.0636 & 0.0615 & 0.1128 & -0.5848 & 0.0016 & 0.1491 & 3.0215 \\
\hline Wednesday & 1.0629 & 0.0617 & 0.1294 & -0.5214 & 0.0016 & 0.1499 & 3.0193 \\
\hline Thursday & 1.0627 & 0.0618 & 0.1464 & -0.4301 & 0.0016 & 0.1503 & 2.9441 \\
\hline Friday & 1.0632 & 0.0625 & 0.1931 & -0.3850 & 0.0016 & 0.1504 & 2.7759 \\
\hline
\end{tabular}

Panel D: EUR

\begin{tabular}{|c|c|c|c|c|c|c|c|}
\hline \multirow[t]{2}{*}{ Day } & \multicolumn{7}{|c|}{ Spot Rate } \\
\hline & Mean & Std. Dev. & Skew & Kurtosis & $\begin{array}{l}\text { Quoted } \\
\text { Spread }\end{array}$ & $\begin{array}{c}\% \text { Quoted } \\
\text { Spread }\end{array}$ & $\mathrm{r}^{2} * 10^{4}$ \\
\hline Monday & 0.7203 & 0.0497 & -0.0644 & -0.8298 & 0.0002 & 0.0284 & 3.4464 \\
\hline Tuesday & 0.7204 & 0.0499 & -0.0256 & -0.8229 & 0.0002 & 0.0288 & 3.3445 \\
\hline Wednesday & 0.7202 & 0.0502 & -0.0266 & -0.8531 & 0.0002 & 0.0282 & 3.3769 \\
\hline Thursday & 0.7200 & 0.0496 & -0.0352 & -0.8977 & 0.0002 & 0.0283 & 2.9355 \\
\hline Friday & 0.7205 & 0.0496 & -0.0418 & -0.8312 & 0.0002 & 0.0287 & 2.8817 \\
\hline \multirow[t]{2}{*}{ Day } & \multicolumn{7}{|c|}{ 90-Day Forward Rate } \\
\hline & Mean & Std. Dev. & Skew & Kurtosis & $\begin{array}{l}\text { Quoted } \\
\text { Spread }\end{array}$ & $\begin{array}{c}\% \text { Quoted } \\
\text { Spread } \\
\end{array}$ & $\mathrm{r}^{2} * 10^{4}$ \\
\hline Monday & 0.7211 & 0.0490 & -0.0403 & -0.8539 & 0.0003 & 0.0465 & 3.4277 \\
\hline Tuesday & 0.7212 & 0.0492 & -0.0006 & -0.8484 & 0.0003 & 0.0451 & 3.3310 \\
\hline Wednesday & 0.7209 & 0.0495 & 0.0004 & -0.8728 & 0.0003 & 0.0456 & 3.3807 \\
\hline Thursday & 0.7208 & 0.0488 & -0.0112 & -0.9176 & 0.0003 & 0.0448 & 2.9509 \\
\hline Friday & 0.7213 & 0.0489 & -0.0168 & -0.8535 & 0.0003 & 0.0453 & 2.8673 \\
\hline
\end{tabular}


Panel E: GBP

\begin{tabular}{|c|c|c|c|c|c|c|c|}
\hline \multirow[t]{2}{*}{ Day } & \multicolumn{7}{|c|}{ Spot Rate } \\
\hline & Mean & Std. Dev. & Skew & Kurtosis & $\begin{array}{l}\text { Quoted } \\
\text { Spread }\end{array}$ & $\begin{array}{c}\% \text { Quoted } \\
\text { Spread } \\
\end{array}$ & $\mathrm{r}^{2} * 10^{4}$ \\
\hline Monday & 0.6126 & 0.0618 & -0.6122 & -0.6930 & 0.0002 & 0.0244 & 3.3893 \\
\hline Tuesday & 0.6120 & 0.0622 & -0.5904 & -0.7317 & 0.0002 & 0.0244 & 3.2980 \\
\hline Wednesday & 0.6118 & 0.0619 & -0.5684 & -0.6945 & 0.0002 & 0.0244 & 3.7779 \\
\hline Thursday & 0.6119 & 0.0618 & -0.6163 & -0.7124 & 0.0002 & 0.0245 & 3.2935 \\
\hline Friday & 0.6122 & 0.0619 & -0.6175 & -0.7048 & 0.0002 & 0.0244 & 3.5920 \\
\hline \multirow[t]{2}{*}{ Day } & \multicolumn{7}{|c|}{ 90-Day Forward Rate } \\
\hline & Mean & Std. Dev. & Skew & Kurtosis & $\begin{array}{l}\text { Quoted } \\
\text { Spread }\end{array}$ & $\begin{array}{c}\% \text { Quoted } \\
\text { Spread }\end{array}$ & $\mathrm{r}^{2} * 10^{4}$ \\
\hline Monday & 0.6137 & 0.0607 & -0.5926 & -0.7003 & 0.0003 & 0.0518 & 3.3402 \\
\hline Tuesday & 0.6130 & 0.0612 & -0.5709 & -0.7384 & 0.0003 & 0.0519 & 3.2500 \\
\hline Wednesday & 0.6128 & 0.0608 & -0.5488 & -0.7002 & 0.0003 & 0.0502 & 3.7285 \\
\hline Thursday & 0.6129 & 0.0607 & -0.5955 & -0.7204 & 0.0003 & 0.0515 & 3.2056 \\
\hline Friday & 0.6132 & 0.0608 & -0.5981 & -0.7104 & 0.0003 & 0.0513 & 3.5343 \\
\hline
\end{tabular}

Panel F: JPY

\begin{tabular}{|c|c|c|c|c|c|c|c|}
\hline \multirow[t]{2}{*}{ Day } & \multicolumn{7}{|c|}{ Spot Rate } \\
\hline & Mean & Std. Dev. & Skew & Kurtosis & $\begin{array}{l}\text { Quoted } \\
\text { Spread }\end{array}$ & $\begin{array}{c}\% \text { Quoted } \\
\text { Spread }\end{array}$ & $\mathrm{r}^{2} * 10^{4}$ \\
\hline Monday & 94.2304 & 8.0232 & 0.2828 & -0.8674 & 0.0598 & 0.0639 & 3.2911 \\
\hline Tuesday & 94.3715 & 8.1522 & 0.2900 & -0.9051 & 0.0599 & 0.0640 & 2.5035 \\
\hline Wednesday & 94.3333 & 8.0786 & 0.3199 & -0.8977 & 0.0596 & 0.0637 & 2.8787 \\
\hline Thursday & 94.3188 & 8.0719 & 0.2919 & -0.9154 & 0.0600 & 0.0641 & 2.4128 \\
\hline Friday & 94.2743 & 8.0637 & 0.3078 & -0.8872 & 0.0597 & 0.0638 & 3.1426 \\
\hline \multirow[t]{2}{*}{ Day } & \multicolumn{7}{|c|}{ 90-Day Forward Rate } \\
\hline & Mean & Std. Dev. & Skew & Kurtosis & $\begin{array}{l}\text { Quoted } \\
\text { Spread }\end{array}$ & $\begin{array}{c}\text { \% Quoted } \\
\text { Spread }\end{array}$ & $\mathrm{r}^{2} * 10^{4}$ \\
\hline Monday & 93.9982 & 7.8295 & 0.2532 & -0.8648 & 0.1200 & 0.1282 & 3.2928 \\
\hline Tuesday & 94.1310 & 7.9437 & 0.2577 & -0.9062 & 0.1195 & 0.1275 & 2.4870 \\
\hline Wednesday & 94.0935 & 7.8718 & 0.2907 & -0.8957 & 0.1191 & 0.1271 & 2.9073 \\
\hline Thursday & 94.0764 & 7.8642 & 0.2630 & -0.9101 & 0.1201 & 0.1281 & 2.4330 \\
\hline Friday & 94.0378 & 7.8645 & 0.2791 & -0.8856 & 0.1196 & 0.1277 & 3.1674 \\
\hline
\end{tabular}




\subsection{End-of-the-Month Effect}

In this section, we investigate the behavior of foreign exchange rates at the end of each month of a year as presented in Table 6. It is likely that at the beginning and end of a year, spot and forward rates are more dispersed than the rest of the year. More specifically, the standard deviations of both spot and forward markets are largest in February for the AUD, CAD, and CHF and in December for the JPY. Interestingly, EUR spot and forward markets are most dispersed in the middle of the year. However, the characteristics of higher moments of each currency are random for each month (timevarying). The skewness and excess kurtosis of spot and forward rates are generally negative, which is consistent with the results of the end-of-the-week effect (as shown in Table 3). It is also noted that the GBP spot and forward rates are right-skewed for most of the year, with the exception of October and November.

The monthly quoted spread and percentage quoted spread for spot and forward rates are relatively constant over a year, whereas forward markets have wider spreads. The size of the spreads by the end-of-month effect and that of end-of-the-week effect is almost identical for spot and forward rates. Thus, quoted spread and percentage quoted spread are not seasonal. 
Table 6 End-of-the-Month Effect Over the Entire Sample Period

This table presents the summary statistics for each month of the Australian dollar (AUD), Canadian dollar (CAD), Swiss franc (CHF), Euro (EUR), British pound (GBP), and Japanese yen (JPY) respectively over entire sample period. The quoted spread is defined as the difference between quoted ask and quoted bid prices. \% quoted spread is equal to $\frac{\text { Quoted Spread }}{\text { Mid Point }} X 100$. The $\mathrm{r}^{2}$ is a proxy of daily realized volatility measure.

Panel A: AUD

\begin{tabular}{|c|c|c|c|c|c|c|c|}
\hline \multirow[t]{2}{*}{ Month } & \multicolumn{7}{|c|}{ Spot Rate } \\
\hline & Mean & Std. Dev. & Skew & Kurtosis & Quoted Spread & \% Quoted Spread & $\mathrm{r}^{2} * 10^{4}$ \\
\hline January & 1.2538 & 0.1623 & 0.4471 & 0.7820 & 0.0008 & 0.0635 & 16.4652 \\
\hline February & 1.2361 & 0.1679 & 0.3571 & 0.2698 & 0.0008 & 0.0638 & 5.0158 \\
\hline March & 1.2667 & 0.1271 & -0.2283 & -1.2980 & 0.0008 & 0.0646 & 14.1743 \\
\hline April & 1.2406 & 0.1220 & -0.4939 & -1.5656 & 0.0007 & 0.0593 & 16.6630 \\
\hline May & 1.2479 & 0.1082 & -0.5801 & 0.2934 & 0.0008 & 0.0613 & 25.2725 \\
\hline June & 1.2483 & 0.1200 & -0.1193 & -0.2255 & 0.0008 & 0.0637 & 2.3698 \\
\hline July & 1.2258 & 0.1192 & 0.2967 & -1.0336 & 0.0008 & 0.0625 & 9.5712 \\
\hline August & 1.2514 & 0.0956 & 0.4505 & -0.8829 & 0.0008 & 0.0648 & 17.0959 \\
\hline September & 1.2261 & 0.1183 & -0.3762 & -1.5947 & 0.0008 & 0.0636 & 34.3848 \\
\hline October & 1.2400 & 0.1611 & 0.1382 & -1.2278 & 0.0008 & 0.0599 & 49.2109 \\
\hline November & 1.2445 & 0.1568 & 0.5469 & -0.2371 & 0.0008 & 0.0622 & 6.9978 \\
\hline December & 1.2252 & 0.1448 & -0.3441 & -0.6353 & 0.0008 & 0.0611 & 13.0950 \\
\hline \multirow[t]{2}{*}{ Month } & \multicolumn{7}{|c|}{ 90-Day Forward Rate } \\
\hline & Mean & Std. Dev. & Skew & Kurtosis & Quoted Spread & \% Quoted Spread & $r^{2} * 10^{4}$ \\
\hline January & 1.2628 & 0.1612 & 0.4802 & 0.8698 & 0.0013 & 0.1036 & 15.6577 \\
\hline February & 1.2452 & 0.1664 & 0.3859 & 0.3674 & 0.0013 & 0.1023 & 4.7227 \\
\hline March & 1.2755 & 0.1250 & -0.2174 & -1.3300 & 0.0013 & 0.1046 & 14.1191 \\
\hline April & 1.2493 & 0.1212 & -0.4075 & -1.5956 & 0.0013 & 0.0997 & 16.6415 \\
\hline May & 1.2565 & 0.1075 & -0.4697 & 0.2881 & 0.0013 & 0.1061 & 24.9671 \\
\hline June & 1.2568 & 0.1196 & -0.0234 & -0.1358 & 0.0013 & 0.1027 & 2.3247 \\
\hline July & 1.2340 & 0.1187 & 0.3984 & -0.8593 & 0.0013 & 0.1012 & 9.5483 \\
\hline August & 1.2597 & 0.0948 & 0.5789 & -0.5897 & 0.0013 & 0.1049 & 16.7918 \\
\hline September & 1.2332 & 0.1172 & -0.3228 & -1.6220 & 0.0013 & 0.1012 & 32.5957 \\
\hline October & 1.2482 & 0.1611 & 0.1847 & -1.1573 & 0.0013 & 0.1026 & 50.6208 \\
\hline November & 1.2518 & 0.1554 & 0.5898 & -0.1648 & 0.0013 & 0.1028 & 6.8842 \\
\hline December & 1.2330 & 0.1437 & -0.2994 & -0.6028 & 0.0013 & 0.1009 & 12.5711 \\
\hline
\end{tabular}


Panel B: CAD

\begin{tabular}{|c|c|c|c|c|c|c|c|}
\hline \multirow[t]{2}{*}{ Month } & \multicolumn{7}{|c|}{ Spot Rate } \\
\hline & Mean & Std. Dev. & Skew & Kurtosis & Quoted Spread & \% Quoted Spread & $\mathrm{r}^{2} * 10^{4}$ \\
\hline January & 1.1491 & 0.1102 & -0.0019 & -1.2314 & 0.0006 & 0.0527 & 3.1744 \\
\hline February & 1.1444 & 0.1257 & -0.0209 & -1.3270 & 0.0006 & 0.0531 & 3.1757 \\
\hline March & 1.1628 & 0.1019 & -0.2579 & -1.1227 & 0.0006 & 0.0520 & 7.9785 \\
\hline April & 1.1531 & 0.1208 & 0.5928 & -0.3594 & 0.0006 & 0.0526 & 13.9030 \\
\hline May & 1.1318 & 0.1203 & 1.1744 & 0.5488 & 0.0006 & 0.0536 & 15.5423 \\
\hline June & 1.1400 & 0.1038 & 0.9459 & 0.2789 & 0.0006 & 0.0530 & 7.7953 \\
\hline July & 1.1259 & 0.1045 & 1.1290 & 0.3012 & 0.0006 & 0.0537 & 9.5288 \\
\hline August & 1.1258 & 0.0873 & 1.6890 & 2.4525 & 0.0006 & 0.0536 & 6.3677 \\
\hline September & 1.1004 & 0.0822 & 0.9058 & 0.6498 & 0.0006 & 0.0548 & 10.0983 \\
\hline October & 1.1103 & 0.0923 & -0.5909 & -0.9111 & 0.0006 & 0.0544 & 27.3954 \\
\hline November & 1.1175 & 0.0833 & -0.0647 & -1.6673 & 0.0006 & 0.0540 & 7.8469 \\
\hline December & 1.1114 & 0.0906 & -0.3658 & -2.2124 & 0.0006 & 0.0544 & 3.1914 \\
\hline \multirow[t]{2}{*}{ Month } & \multicolumn{7}{|c|}{ 90-Day Forward Rate } \\
\hline & Mean & Std. Dev. & Skew & Kurtosis & Quoted Spread & \% Quoted Spread & $\mathrm{r}^{2} * 10^{4}$ \\
\hline January & 1.1494 & 0.1104 & 0.0640 & -1.1608 & 0.0012 & 0.1054 & 3.2491 \\
\hline February & 1.1444 & 0.1256 & 0.0337 & -1.2757 & 0.0012 & 0.1050 & 3.1234 \\
\hline March & 1.1624 & 0.1020 & -0.1894 & -1.0856 & 0.0012 & 0.1026 & 8.0162 \\
\hline April & 1.1525 & 0.1215 & 0.6452 & -0.2648 & 0.0012 & 0.1038 & 13.8223 \\
\hline May & 1.1312 & 0.1210 & 1.2152 & 0.6717 & 0.0012 & 0.1084 & 15.5049 \\
\hline June & 1.1393 & 0.1040 & 0.9938 & 0.4094 & 0.0012 & 0.1062 & 7.8777 \\
\hline July & 1.1253 & 0.1044 & 1.1827 & 0.4829 & 0.0012 & 0.1101 & 9.4104 \\
\hline August & 1.1253 & 0.0875 & 1.7367 & 2.6819 & 0.0012 & 0.1059 & 6.3577 \\
\hline September & 1.0996 & 0.0824 & 0.9659 & 0.8782 & 0.0012 & 0.1084 & 9.9927 \\
\hline October & 1.1098 & 0.0918 & -0.5735 & -0.8387 & 0.0012 & 0.1089 & 27.6298 \\
\hline November & 1.1168 & 0.0826 & -0.0481 & -1.6280 & 0.0012 & 0.1067 & 7.8727 \\
\hline December & 1.1108 & 0.0899 & -0.3455 & -2.1876 & 0.0012 & 0.1074 & 3.1123 \\
\hline
\end{tabular}


Panel C: CHF

\begin{tabular}{|c|c|c|c|c|c|c|c|}
\hline \multirow[t]{2}{*}{ Month } & \multicolumn{7}{|c|}{ Spot Rate } \\
\hline & Mean & Std. Dev. & Skew & Kurtosis & Quoted Spread & \% Quoted Spread & $r^{2} * 10^{4}$ \\
\hline January & 1.1524 & 0.1085 & -0.7948 & -0.1197 & 0.0006 & 0.0526 & 17.9019 \\
\hline February & 1.1462 & 0.1169 & -0.4969 & -0.2722 & 0.0006 & 0.0529 & 4.4471 \\
\hline March & 1.1664 & 0.1043 & -0.4953 & -0.8737 & 0.0006 & 0.0519 & 6.4240 \\
\hline April & 1.1708 & 0.0835 & -0.1812 & -0.8895 & 0.0006 & 0.0515 & 8.5885 \\
\hline May & 1.1722 & 0.0794 & -0.7740 & -1.3468 & 0.0006 & 0.0514 & 17.9579 \\
\hline June & 1.1665 & 0.0948 & -0.3836 & -1.9726 & 0.0006 & 0.0518 & 9.1814 \\
\hline July & 1.1655 & 0.1022 & -0.1412 & -2.4140 & 0.0006 & 0.0519 & 4.2228 \\
\hline August & 1.1620 & 0.0939 & -0.4800 & -1.9080 & 0.0006 & 0.0520 & 5.8091 \\
\hline September & 1.1554 & 0.1079 & -0.4388 & -1.3411 & 0.0006 & 0.0524 & 6.3897 \\
\hline October & 1.1512 & 0.1025 & -0.5353 & -0.8499 & 0.0006 & 0.0526 & 4.1075 \\
\hline November & 1.1435 & 0.1049 & 0.0176 & -0.6709 & 0.0006 & 0.0529 & 11.2852 \\
\hline December & 1.1215 & 0.1151 & 0.1089 & 0.0719 & 0.0006 & 0.0541 & 32.3777 \\
\hline \multirow[t]{2}{*}{ Month } & \multicolumn{7}{|c|}{ 90-Day Forward Rate } \\
\hline & Mean & Std. Dev. & Skew & Kurtosis & Quoted Spread & \% Quoted Spread & $r^{2} * 10^{4}$ \\
\hline January & 1.1482 & 0.1056 & -0.8383 & -0.0004 & 0.0016 & 0.1426 & 17.3408 \\
\hline February & 1.1418 & 0.1138 & -0.5306 & -0.2089 & 0.0016 & 0.1436 & 4.3938 \\
\hline March & 1.1615 & 0.1009 & -0.5026 & -0.8688 & 0.0016 & 0.1385 & 6.2853 \\
\hline April & 1.1659 & 0.0812 & -0.0941 & -0.6490 & 0.0016 & 0.1391 & 8.4267 \\
\hline May & 1.1669 & 0.0766 & -0.7455 & -1.2916 & 0.0016 & 0.1389 & 17.6494 \\
\hline June & 1.1613 & 0.0916 & -0.3672 & -1.9285 & 0.0016 & 0.1398 & 8.9987 \\
\hline July & 1.1603 & 0.0989 & -0.1063 & -2.3598 & 0.0016 & 0.1425 & 4.1329 \\
\hline August & 1.1568 & 0.0906 & -0.4651 & -1.8163 & 0.0016 & 0.1415 & 5.8095 \\
\hline September & 1.1490 & 0.1048 & -0.4120 & -1.3960 & 0.0016 & 0.1427 & 6.0331 \\
\hline October & 1.1460 & 0.0989 & -0.5735 & -0.8226 & 0.0016 & 0.1430 & 4.8872 \\
\hline November & 1.1375 & 0.1015 & 0.0316 & -0.6620 & 0.0016 & 0.1441 & 10.8495 \\
\hline December & 1.1166 & 0.1113 & 0.0783 & 0.1649 & 0.0016 & 0.1470 & 30.7110 \\
\hline
\end{tabular}


Panel D: EUR

\begin{tabular}{|c|c|c|c|c|c|c|c|}
\hline \multirow[t]{2}{*}{ Month } & \multicolumn{7}{|c|}{ Spot Rate } \\
\hline & Mean & Std. Dev. & Skew & Kurtosis & Quoted Spread & \% Quoted Spread & $\mathrm{r}^{2} * 10^{4}$ \\
\hline January & 0.7582 & 0.0448 & -0.5637 & -0.0633 & 0.0002 & 0.0296 & 16.2912 \\
\hline February & 0.7572 & 0.0511 & -0.4026 & 0.6710 & 0.0003 & 0.0328 & 2.4306 \\
\hline March & 0.7548 & 0.0582 & -1.1827 & 2.2320 & 0.0002 & 0.0298 & 6.5458 \\
\hline April & 0.7548 & 0.0553 & -0.9505 & 2.1136 & 0.0002 & 0.0285 & 4.7394 \\
\hline May & 0.7588 & 0.0602 & -1.0156 & 0.1470 & 0.0002 & 0.0300 & 17.3540 \\
\hline June & 0.7615 & 0.0652 & -1.0016 & 0.2267 & 0.0002 & 0.0294 & 0.9795 \\
\hline July & 0.7539 & 0.0635 & -0.5330 & -0.4997 & 0.0002 & 0.0302 & 6.4058 \\
\hline August & 0.7589 & 0.0509 & -0.4236 & -1.7141 & 0.0002 & 0.0318 & 7.4102 \\
\hline September & 0.7501 & 0.0530 & 0.2941 & -1.8431 & 0.0002 & 0.0285 & 14.2437 \\
\hline October & 0.7534 & 0.0534 & -0.1216 & -1.4720 & 0.0002 & 0.0302 & 16.4616 \\
\hline November & 0.7515 & 0.0576 & 0.0068 & -0.2133 & 0.0002 & 0.0321 & 11.5523 \\
\hline December & 0.7415 & 0.0486 & 1.3680 & 2.4896 & 0.0002 & 0.0307 & 17.3191 \\
\hline \multirow[t]{2}{*}{ Month } & \multicolumn{7}{|c|}{ 90-Day Forward Rate } \\
\hline & Mean & Std. Dev. & Skew & Kurtosis & Quoted Spread & \% Quoted Spread & $\mathrm{r}^{2} * 10^{4}$ \\
\hline January & 0.7579 & 0.0437 & -0.5627 & -0.1646 & 0.0004 & 0.0503 & 15.5973 \\
\hline February & 0.7567 & 0.0499 & -0.4107 & 0.5204 & 0.0004 & 0.0506 & 2.3908 \\
\hline March & 0.7542 & 0.0569 & -1.1500 & 2.1137 & 0.0004 & 0.0516 & 6.4326 \\
\hline April & 0.7542 & 0.0546 & -0.8170 & 2.0170 & 0.0004 & 0.0539 & 4.7609 \\
\hline May & 0.7580 & 0.0591 & -0.9307 & -0.0127 & 0.0004 & 0.0532 & 17.0905 \\
\hline June & 0.7607 & 0.0640 & -0.9348 & 0.0605 & 0.0004 & 0.0539 & 0.9411 \\
\hline July & 0.7531 & 0.0622 & -0.4612 & -0.5816 & 0.0004 & 0.0473 & 6.3271 \\
\hline August & 0.7581 & 0.0498 & -0.3450 & -1.7832 & 0.0004 & 0.0489 & 7.3834 \\
\hline September & 0.7484 & 0.0522 & 0.2875 & -1.9026 & 0.0004 & 0.0474 & 13.1540 \\
\hline October & 0.7525 & 0.0524 & -0.1688 & -1.5649 & 0.0004 & 0.0495 & 18.3350 \\
\hline November & 0.7503 & 0.0567 & -0.0341 & -0.3419 & 0.0004 & 0.0474 & 11.5856 \\
\hline December & 0.7407 & 0.0471 & 1.3377 & 2.4935 & 0.0004 & 0.0490 & 16.6816 \\
\hline
\end{tabular}


Panel E: GBP

\begin{tabular}{|c|c|c|c|c|c|c|c|}
\hline \multirow[t]{2}{*}{ Month } & \multicolumn{7}{|c|}{ Spot Rate } \\
\hline & Mean & Std. Dev. & Skew & Kurtosis & Quoted Spread & \% Quoted Spread & $\mathrm{r}^{2} * 10^{4}$ \\
\hline January & 0.5742 & 0.0624 & 0.7720 & -0.3837 & 0.0002 & 0.0271 & 3.9983 \\
\hline February & 0.5765 & 0.0687 & 0.7285 & -0.9636 & 0.0002 & 0.0271 & 5.1861 \\
\hline March & 0.5739 & 0.0706 & 0.9516 & -0.6738 & 0.0002 & 0.0277 & 0.8718 \\
\hline April & 0.5670 & 0.0653 & 0.8451 & -1.0464 & 0.0002 & 0.0274 & 7.4833 \\
\hline May & 0.5639 & 0.0624 & 1.3279 & 1.1442 & 0.0002 & 0.0272 & 20.9203 \\
\hline June & 0.5610 & 0.0555 & 0.9596 & 0.5897 & 0.0002 & 0.0272 & 3.0611 \\
\hline July & 0.5550 & 0.0474 & 0.4159 & -0.5654 & 0.0002 & 0.0269 & 4.7358 \\
\hline August & 0.5637 & 0.0488 & 0.6872 & 0.0536 & 0.0002 & 0.0272 & 13.7037 \\
\hline September & 0.5660 & 0.0469 & 0.1210 & -0.3758 & 0.0002 & 0.0274 & 3.0983 \\
\hline October & 0.5661 & 0.0493 & -0.4790 & -0.9877 & 0.0001 & 0.0258 & 15.4126 \\
\hline November & 0.5715 & 0.0617 & -0.0165 & -1.9542 & 0.0001 & 0.0260 & 10.5266 \\
\hline December & 0.5802 & 0.0651 & 0.2675 & -1.6633 & 0.0002 & 0.0264 & 5.2295 \\
\hline \multirow[t]{2}{*}{ Month } & \multicolumn{7}{|c|}{ 90-Day Forward Rate } \\
\hline & Mean & Std. Dev. & Skew & Kurtosis & Quoted Spread & \% Quoted Spread & $\mathrm{r}^{2} * 10^{4}$ \\
\hline January & 0.5756 & 0.0617 & 0.7755 & -0.3363 & 0.0003 & 0.0533 & 3.9568 \\
\hline February & 0.5778 & 0.0678 & 0.7375 & -0.9312 & 0.0003 & 0.0524 & 5.1387 \\
\hline March & 0.5753 & 0.0695 & 0.9540 & -0.6467 & 0.0003 & 0.0573 & 0.8553 \\
\hline April & 0.5684 & 0.0647 & 0.8250 & -1.0780 & 0.0003 & 0.0574 & 7.4958 \\
\hline May & 0.5653 & 0.0617 & 1.3157 & 1.1341 & 0.0003 & 0.0578 & 20.7018 \\
\hline June & 0.5623 & 0.0549 & 0.9495 & 0.5910 & 0.0003 & 0.0530 & 2.9594 \\
\hline July & 0.5563 & 0.0469 & 0.3903 & -0.5608 & 0.0003 & 0.0571 & 4.6318 \\
\hline August & 0.5651 & 0.0486 & 0.6247 & 0.0278 & 0.0003 & 0.0563 & 13.6350 \\
\hline September & 0.5666 & 0.0467 & 0.1194 & -0.3805 & 0.0003 & 0.0530 & 2.7052 \\
\hline October & 0.5674 & 0.0493 & -0.4823 & -0.9946 & 0.0004 & 0.0700 & 17.3746 \\
\hline November & 0.5722 & 0.0612 & -0.0221 & -1.9405 & 0.0003 & 0.0511 & 9.7393 \\
\hline December & 0.5810 & 0.0649 & 0.2798 & -1.6180 & 0.0003 & 0.0509 & 5.4536 \\
\hline
\end{tabular}


Panel F: JPY

\begin{tabular}{|c|c|c|c|c|c|c|c|}
\hline \multirow[t]{2}{*}{ Month } & \multicolumn{7}{|c|}{ Spot Rate } \\
\hline & Mean & Std. Dev. & Skew & Kurtosis & Quoted Spread & \% Quoted Spread & $r^{2} * 10^{4}$ \\
\hline January & 102.0038 & 12.7229 & -0.0879 & -1.1290 & 0.0600 & 0.0598 & 5.3854 \\
\hline February & 102.6075 & 11.8062 & -0.4710 & -0.6411 & 0.0600 & 0.0593 & 12.0863 \\
\hline March & 105.5214 & 8.5937 & 0.3757 & -1.2040 & 0.0600 & 0.0572 & 11.3314 \\
\hline April & 106.5429 & 8.0591 & 0.0659 & -0.6598 & 0.0600 & 0.0566 & 11.0059 \\
\hline May & 106.2814 & 9.6341 & -0.1912 & -0.2329 & 0.0600 & 0.0569 & 5.6621 \\
\hline June & 107.0486 & 10.6678 & -0.4218 & -0.0358 & 0.0600 & 0.0566 & 2.9881 \\
\hline July & 106.5871 & 10.8841 & -1.0377 & -0.0789 & 0.0600 & 0.0569 & 4.0765 \\
\hline August & 105.5957 & 11.3068 & -1.1145 & 0.1452 & 0.0600 & 0.0576 & 4.1318 \\
\hline September & 105.0143 & 12.3378 & -0.9703 & -0.7236 & 0.0600 & 0.0580 & 5.2193 \\
\hline October & 103.3671 & 13.1886 & -0.6574 & -1.0278 & 0.0600 & 0.0591 & 12.1753 \\
\hline November & 102.1014 & 13.1556 & -0.1605 & -1.7414 & 0.0600 & 0.0598 & 10.6993 \\
\hline December & 102.3500 & 13.5202 & -0.1888 & -1.5830 & 0.0600 & 0.0597 & 14.9672 \\
\hline \multirow[t]{2}{*}{ Month } & \multicolumn{7}{|c|}{ 90-Day Forward Rate } \\
\hline & Mean & Std. Dev. & Skew & Kurtosis & Quoted Spread & \% Quoted Spread & $r^{2} * 10^{4}$ \\
\hline January & 101.4256 & 12.2533 & -0.1273 & -1.1265 & 0.1238 & 0.1226 & 4.8650 \\
\hline February & 102.0225 & 11.3606 & -0.5250 & -0.5971 & 0.1225 & 0.1203 & 12.0851 \\
\hline March & 104.8629 & 8.0855 & 0.3510 & -1.1906 & 0.1343 & 0.1286 & 11.2546 \\
\hline April & 105.8550 & 7.6219 & 0.0496 & -0.6681 & 0.1329 & 0.1259 & 10.9423 \\
\hline May & 105.5836 & 9.1858 & -0.2133 & -0.1791 & 0.1329 & 0.1266 & 5.6871 \\
\hline June & 106.3207 & 10.1989 & -0.4580 & 0.0870 & 0.1329 & 0.1259 & 2.9774 \\
\hline July & 105.8650 & 10.4214 & -1.0814 & 0.0320 & 0.1357 & 0.1291 & 3.9715 \\
\hline August & 104.8657 & 10.8286 & -1.1749 & 0.2961 & 0.1343 & 0.1293 & 4.2533 \\
\hline September & 104.1536 & 11.8446 & -0.9692 & -0.7237 & 0.1243 & 0.1193 & 5.8455 \\
\hline October & 102.6529 & 12.6767 & -0.6931 & -0.9373 & 0.1257 & 0.1230 & 10.3795 \\
\hline November & 101.3893 & 12.6382 & -0.1670 & -1.7083 & 0.1243 & 0.1224 & 10.6453 \\
\hline December & 101.6764 & 12.9872 & -0.2132 & -1.5161 & 0.1243 & 0.1230 & 14.6520 \\
\hline
\end{tabular}




\subsection{Volatility}

The importance of volatility in financial economics is well documented. For our purpose, it is important to model and analyze it since it imparts significant information on the behavior of the foreign exchange markets. Volatility and market behavior (liquidity and market depth) are connected; when liquidity disappears, then volatility amplifies. Thus, it is interesting to examine the time series behavior of volatility in foreign exchange markets.

\subsubsection{Volatility Pattern on Day-of-the-Week}

Figure 1 presents the intertemporal volatility pattern of currency spot rates on each day of the week over the full sample, non-crisis, and crisis periods, respectively. The return squared $\left(\mathrm{r}^{2}\right)$ is calculated as a proxy of daily realized volatility. In general, the volatility patterns of spot currencies are relatively stable on each day of a week for full sample and non-crisis periods, with the exception of the JPY. However, each currency displays a unique volatility pattern within each period.

The AUD volatility increases on Thursdays and slightly decreases on Fridays over the non-crisis period. The pattern of the AUD volatility over the crisis period displays completely differently as it represents as W-shaped. The highest volatility occurs on Mondays and Fridays, while the lowest volatility is on Tuesdays and Thursdays. The shape of the CAD volatility is hump-shaped, the hump being on Tuesdays. It is interesting to note that this hump on Tuesdays is amplified over the crisis period. The EUR volatility points downward on Thursdays and Fridays over the crisis period, but upward on Thursdays and Fridays over the non-crisis period. The pattern of the GBP 
volatility is similar to that of the CAD volatility, but the hump of the GBP volatility exists on Wednesdays. The structure of the JPY volatility is different from that of the other currencies. The volatility over the non-crisis period represents a flat U-shaped. The lowest volatility happens on Wednesdays. However, the volatility pattern turns into a flat W-shaped for the full sample period and a true W-shape over the crisis period.

Figure 1 The Volatility Pattern on Day-of-the-Week
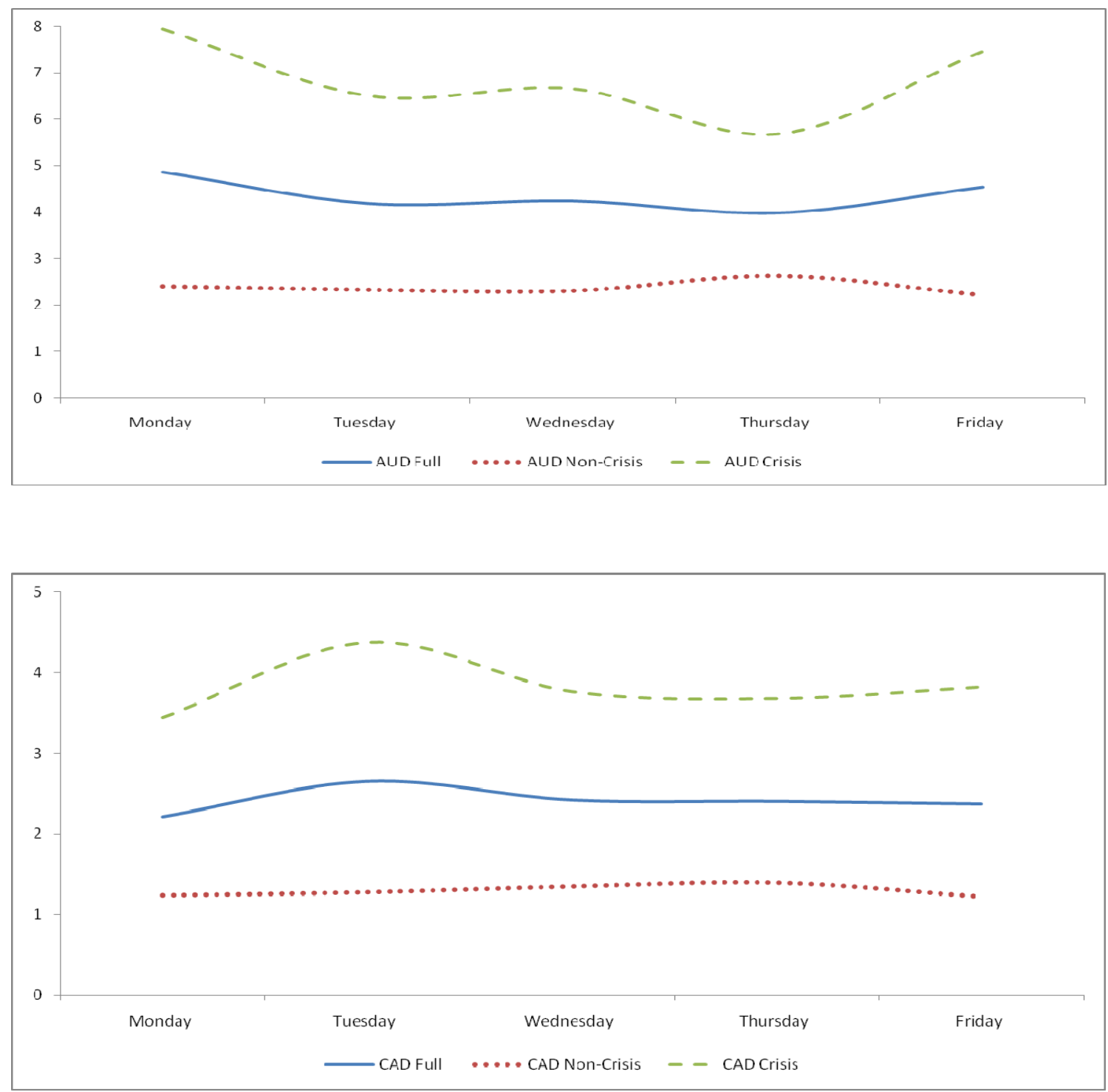
Figure 1 (Continued)
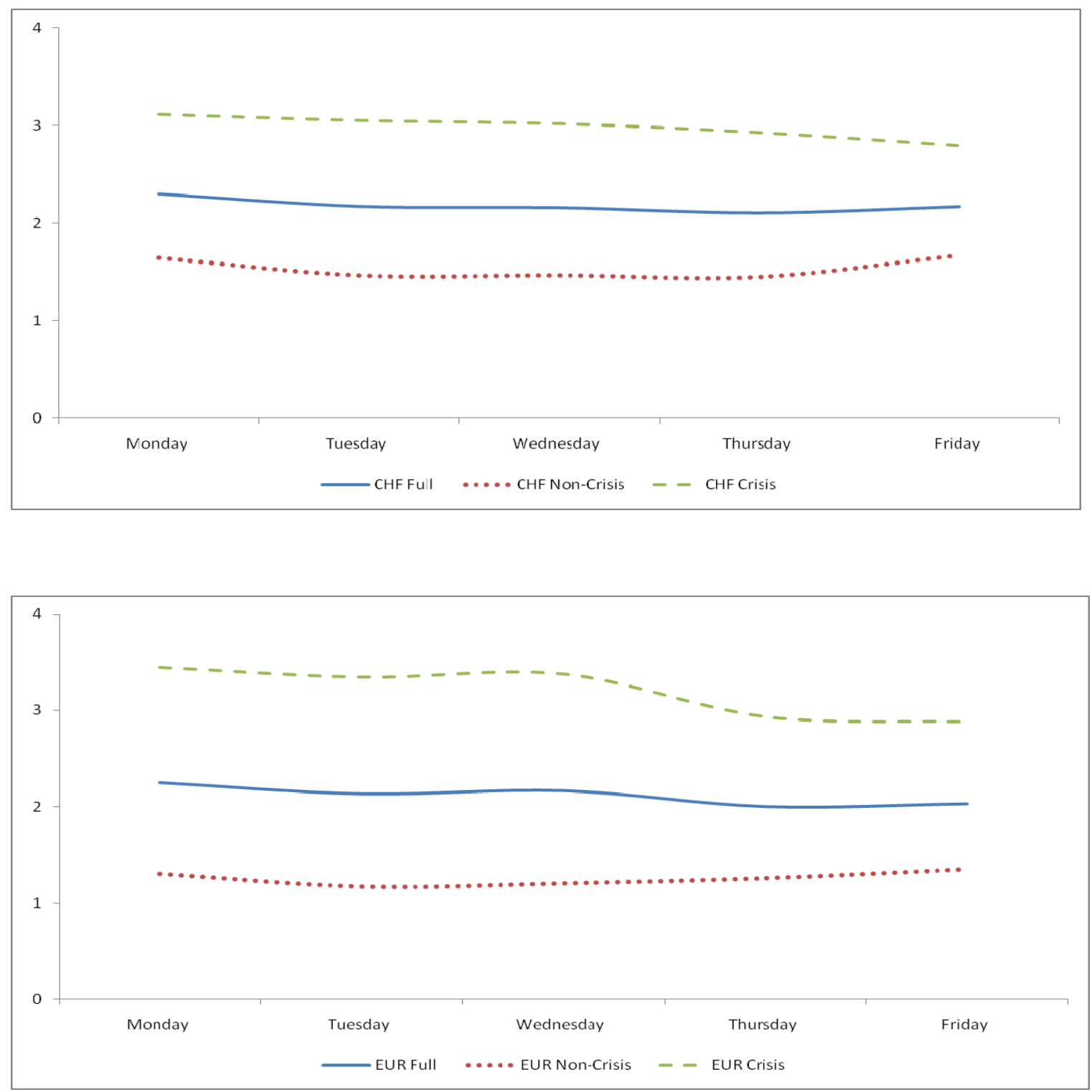
Figure 1 (Continued)
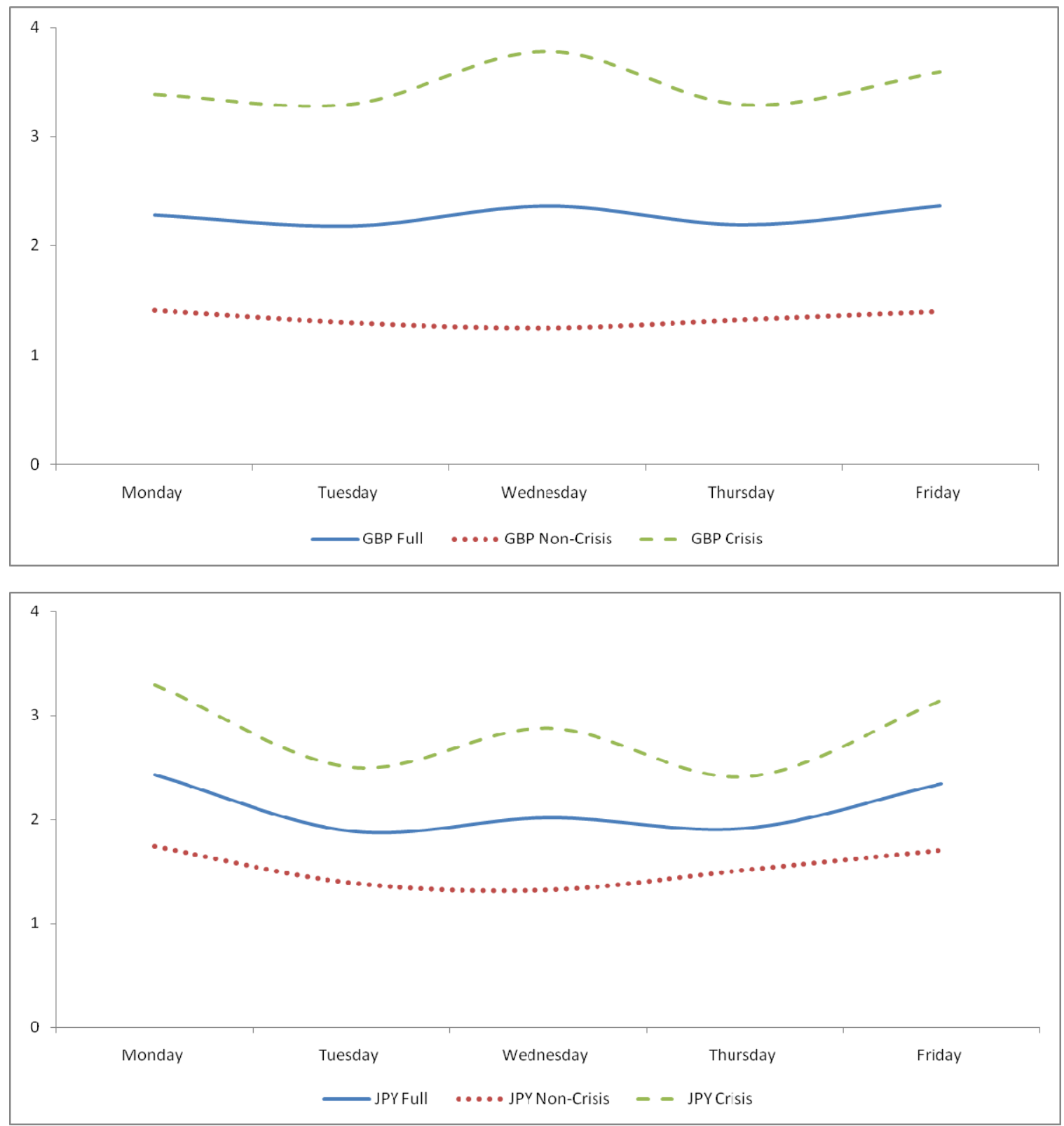


\subsubsection{Volatility Pattern on End-of-the-Month}

Figure 2 displays the volatility pattern for each month of the year for spot currencies over the full sample period. We group the currencies with similar volatility patterns. Panel A displays the intertemporal volatility pattern of the AUD, CAD, and EUR. The shape of the volatilities are two-humped; with humps being in May and October. The most (least) volatile period is October (June). The AUD spot is most volatile in our sample. Generally, spot markets at the beginning and end of a year are more volatile than in the other months, with the exception of the CAD.

Panel B displays the volatility pattern for the spot CHF, GBP, and JPY. The volatility pattern of the CHF appears W-shaped, with large volatility at the beginning, mid-, and end of the year. There is a big jump in CHF volatility in December. These results are opposite to the evidence from the end-of-the-week volatility (as displayed in Figure 1). . The volatility shape of the GBP is three-humps. The humps exist in May, August, and October, with the largest hump in May. For the JPY, the pattern is more a flat U-shaped, although the volatility in January is relatively slow. 
Figure 2 The Volatility Pattern on End-of-the-Month

Panel A: AUD, CAD, and EUR

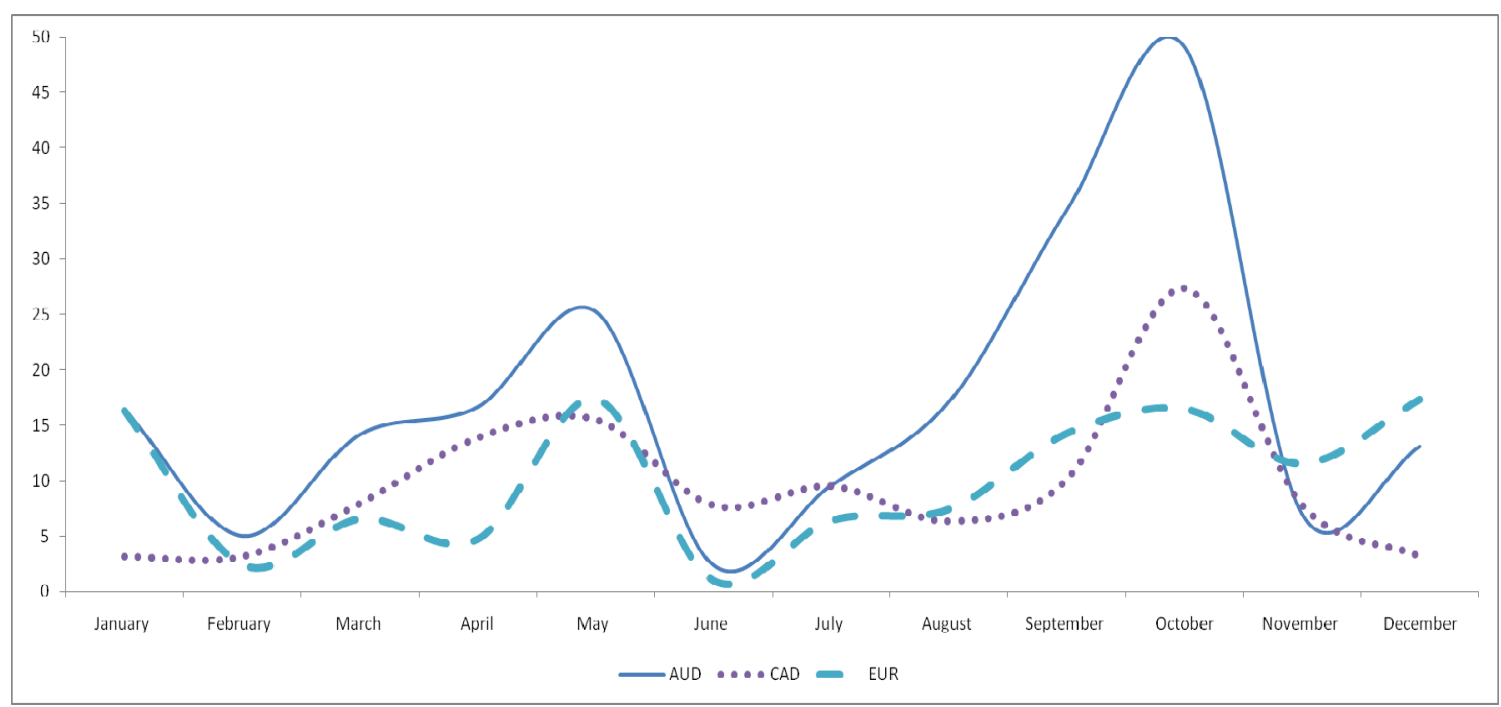

Panel B: CHF, GBP, and JPY

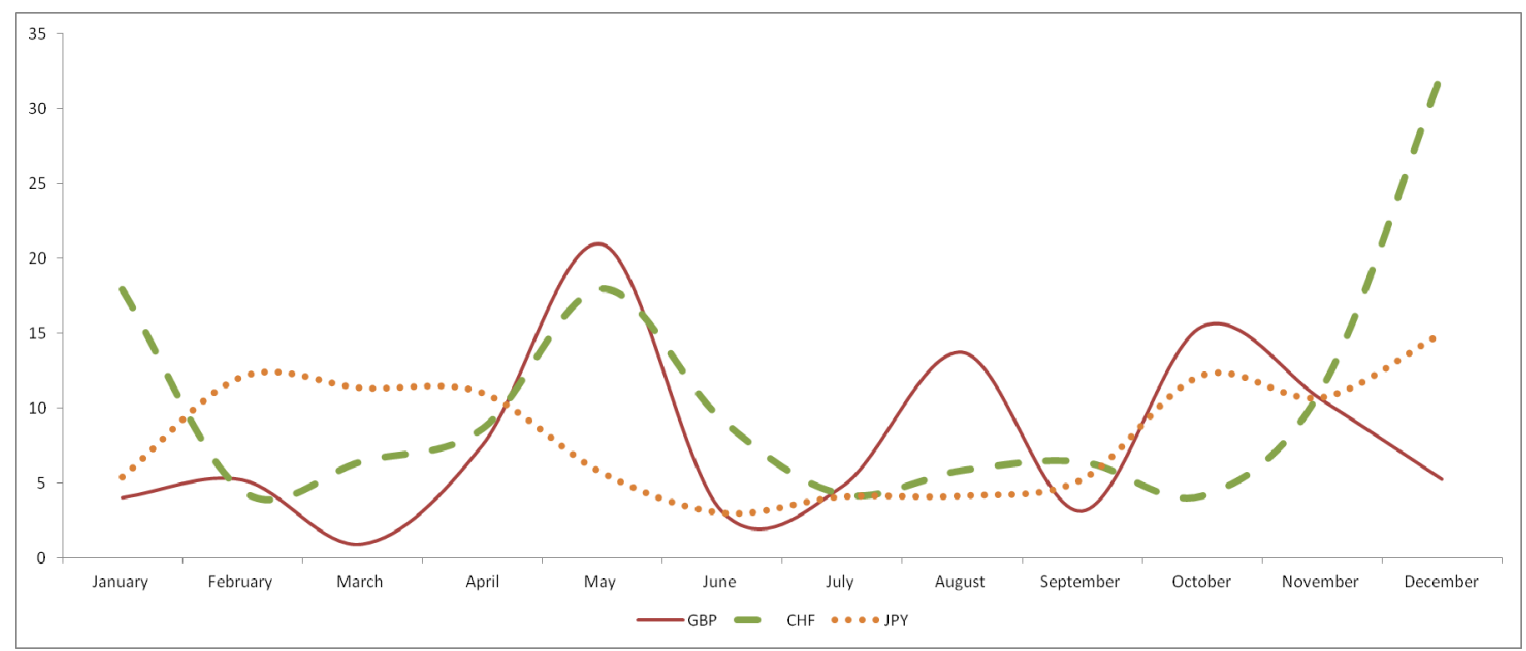




\subsection{Summary and Conclusions}

This chapter has examined some basic but notable aspects of six major actively traded currencies, namely the Australian dollar (AUD), Canadian dollar (CAD), Swiss franc (CHF), Euro (EUR), British pound (GBP), and Japanese yen (JPY) over the full sample, non-crisis, and crisis periods. All currencies are quoted against the United States dollar (USD). The first four-moment characteristics of the currencies' spot and their corresponding forward markets are relatively similar, especially the standard deviation. However, the currencies' spot and their corresponding forward rates are more variant over the non-crisis period, with the exception of the EUR and GBP. The difference in standard deviations between spot and corresponding forward markets is still small. The USD is weakened during the crisis period, and during this period the standard deviations of all currencies are substantially higher. It is interesting to note that the standard deviation of the CAD during the crisis period is smaller than the non-crisis period. In general, higher moments of all currencies over the crisis period are distinguishable from the non-crisis period. All of the currencies' spot and forward rates do not follow a normal distribution irrespectively of time-period considered. Both parametric (paired t-test) and nonparametric (Wilcoxon signed test) statistical tests strongly reject the null hypothesis of the equality of means between the non-crisis and crisis periods. However, the Wilcoxon signed test does accept the equality of means for the GBP forward rate. In sum, the two periods are behaving differently.

Most currencies over the full sample period are most dispersed on Mondays and Fridays. The CAD and EUR are most volatile on Wednesdays. The spreads in forward markets are wider than in corresponding spot markets and are relatively constant over the 
entire week. The standard deviations on each day of forward markets are minimally lower than those of spot markets over the crisis period, but slightly higher over the non-crisis period. Interestingly, the behaviors of higher moments of spot and forward markets during the non-crisis and crisis period are inconclusive. The behaviors of spreads in these two periods are not significantly different from those of the full sample period. The spreads in forward markets are still larger than in corresponding spot markets.

The currencies' spot and forward markets are also more dispersed at the beginning (the AUD, CAD, and CHF) and end (the JPY) of a year. However, the EUR are most dispersed in the middle of a year. In sum, the higher moment factors of all currencies are time-varying, behave differently from month to month, and are consistent with the results of the end-of-the week effect. However, the spreads for both spot and forward rates are relatively invariant over a year; thus they do not seem to be seasonal. It is also important to note that the size of the spread at the end of the month is almost identical with that of the end of the week.

The return squared is employed as a proxy of realized volatility. In general, the volatility patterns of spot currencies are relatively stable over a weekly interval, with the exception of the JPY; however, each currency possesses a unique volatility style. For monthly intervals, the currencies' spot markets at the beginning and end of a year are more volatile than the other months, with the exception of the CAD. The volatility pattern over a monthly interval still displays differently from currency to currency: two-humped for the AUD, CAD, and EUR, W-shaped for the CHF, three-humped for the GBP, and flat U-shaped for the JPY. 
CHAPTER 3: EFFICIENCY OF THE FOREX MARKET BEFORE AND DURING THE CRISIS (DAILY DATA)

In this chapter we examine and compare the efficiency of major currencies before and during the crisis by using daily data. The chapter consists of data description, methodology, and empirical evidence.

\subsection{Data Description}

The main data source is DataStream. We collect bid and ask nominal exchange rates and 90-day forward rates for six major currencies: the Australian dollar (AUD), Canadian dollar (CAD), Swiss franc (CHF), Euro (EUR), British pound (GBP), and Japanese yen (JPY). All currencies are compared against the United States dollar (USD). The entire data period spans from January 2004 to February 2011.

In order to identify the pre-recession (non-crisis) period, we follow the announcement by the Business Cycle Dating Committee, National Bureau of Economic Research (NBER), which defined the U.S. economy as entering a recession in December 2007 (see Labonte (2009)), though stock prices started declining not until the fall of 2008. For our analysis, the non-crisis period starts from 2004 until 2007, totaling four years, and the rest is defined as the crisis period.

\subsection{Methodologies}

There are several methodologies that yield to empirical applications within the themes of the efficiency. 


\subsubsection{Forward Rate as an Unbiased Predictor of Future Spot Rate}

The hypothesis of forward rate as an unbiased predictor of future spot rate states that forward rate fully reflects available information about exchange rate expectations under perfect capital market assumptions, or the average deviation between today's forward rate and future spot rate approaches zero. When we relax the perfect capital market assumption, it is clear that the forward rate unbiased condition depends on two further assumptions:

Market Efficiency: $E\left(\tilde{S}_{t+1}\right)=S_{t+1} \quad$ and

Forward Rate Pricing: $F_{t, 1}=E\left(\tilde{S}_{t+1}\right)$

Thus, for the test of efficiency, we can run ordinary least square regression between spot and forward exchange rate in terms of level and percentage changes (forward premium) as follows:

Using level of spot and forward exchange rates:

$$
S_{i, t+90}=\alpha_{i}+\beta_{i} F_{i, t}^{90}+\varepsilon_{i, t}
$$

Using change in spot and forward exchange rates:

$$
s_{i, t}=\delta_{i}+\theta_{i} f_{i, t}+\varepsilon_{i, t}
$$

where $s_{i, t}=\operatorname{Ln}\left(S_{i, t}\right)-\operatorname{Ln}\left(S_{i, t-1}\right)$ and $f_{i, t}=\operatorname{Ln}\left(F_{i, t}^{90}\right)-\operatorname{Ln}\left(S_{i, t}\right) . S_{i, t}$ and $F_{i, t}^{90}$ are spot and 90-day forward rates. $i$ refers to the Australian dollar (AUD), Canadian dollar (CAD), Swiss franc (CHF), Euro (EUR), British pound (GBP), and Japanese yen (JPY), respectively. $L n$ denotes natural logarithms. To support the hypothesis, the intercept and coefficient approach zero and one, respectively. 


\subsubsection{GARCH $(1,1)$ Specification Model}

We further analyze the relations (1) and (2) by taking the problems of autocorrelation and heteroskedasticity into consideration. In this regard, the generalized autoregressive conditional heteroskedastic (GARCH) is employed. The efficiency results of the FOREX market are obtained by univariate tests (level and change) and GARCH models are compared and analyzed. The GARCH $(1,1)$ specification model of the univariate regression is given as follows:

$$
\begin{aligned}
& \text { Change: } s_{i, t}=\delta_{i}+\theta_{i} f_{i, t}+\varepsilon_{i, t} \\
& \text { where } \varepsilon_{t} \mid \Omega_{t-1} \sim N\left(0, h_{t}\right) \\
& \text { and } h_{t}=\beta_{0}+\beta_{1} h_{t-1}+\beta_{2} \varepsilon_{t-1}^{2}
\end{aligned}
$$

where $\varepsilon_{t}$ is the innovation term, $h_{t}$ is the conditional variance, and $\beta_{0}, \beta_{1}$, and $\beta_{2}$ are constant parameters.

Next, we test the equality of the two relations of the GARCH $(1,1)$ specification by assigning a dummy variable. The purpose of this test does not show whether the theory holds or not, but whether or not the relation between changes in spot and forward premiums remains the same over non-crisis and crisis periods.

\subsubsection{Dummy Variable Models}

In this section we further examine whether the magnitude of a regression coefficient should be bigger for one group than for the other. We test for the difference in the intercepts and slopes, as well as both intercepts and slopes together for the GARCH $(1,1)$ model. The joint test between the intercepts and coefficients of the two comparable 
regression models is widely known as "the Chow Test." The Chow test is employed to test whether or not the two regression models are the same by assuming equal variances (pooling) or whether there is a structural break in the time series data. Dummy variables $\left(D_{t}\right)$ are binary $(0$ and 1$)$, where " 1 " is assigned for the "non-crisis period" and otherwise "0." The model is given as follows:

$$
s_{i, t}=\beta_{1, i}+\beta_{2, i} f_{i, t}+\beta_{3, i} D_{t}+\beta_{4, i} D_{t} f_{i, t}+\varepsilon_{i, t}
$$

The null and alternative hypotheses for intercept testing are as follows:

$$
\begin{aligned}
& H_{0}: \beta_{3}=0 \text { or } \delta_{\text {non-crisis }}=\delta_{\text {crisis }} \\
& H_{1}: \beta_{3} \neq 0 \text { or } \delta_{\text {non-crisis }} \neq \delta_{\text {crisis }}
\end{aligned}
$$

The null and alternative hypotheses for coefficient testing are as follows:

$$
\begin{aligned}
& H_{0}: \beta_{4}=0 \text { or } \theta_{\text {non-crisis }}=\theta_{\text {crisis }} \\
& H_{1}: \beta_{4} \neq 0 \text { or } \theta_{\text {non-crisis }} \neq \theta_{\text {crisis }}
\end{aligned}
$$

The null and alternative hypotheses for both intercept and coefficient testing are as follows:

$$
\begin{aligned}
& H_{0}: \beta_{3}=\beta_{4}=0 \text { or } \delta_{\text {non-crisis }}=\delta_{\text {crisis }} \text { and } \theta_{\text {non-crisis }}=\theta_{\text {crisis }} \\
& H_{1}: \text { Otherwise }
\end{aligned}
$$

The tests of independent intercept and coefficient follow the student- $t$ distribution with $n-4$ degrees of freedom. The Chow test (joint test) follows F-distribution with $n-4$ degrees of freedom. 


\subsubsection{Cointegration Method}

Engle and Granger (1987) introduced a two-step procedure to demonstrate the cointegration relationship by regressing the first time series $\left(y_{t}\right)$ on the second time series $\left(x_{t}\right)$, where both variables are nonstationary.

$$
y_{t}=\mu+b x_{t}+z_{t}
$$

where $\mu$ is the intercept, $b$ is the cointegrating parameter, and $z_{t}$ is the error series. Generally, any linear combination of two time series is also nonstationary. Thus, the stationary test of the error series $\left(z_{t}\right)$ is an indicator of the cointegration. If $y_{t}$ and $x_{t}$ are

cointegrated, $z_{t}$ is stationary. On the other hand, if the null hypothesis of nonstationarity is rejected, the two times series are cointegrated, which is equivalent to rejecting the null hypothesis of no cointegration.

The aforementioned equation is considered a long run or equilibrium relationship, and so is the error series $\left(z_{t}\right)$. The error equilibrium happens randomly and unsystematically, which indicates deviations from the long term trend.

The Granger representation theorem shows that the error correction model and cointegration are equivalent representations, given any set of $I(1)$ variables. Following Johansen (1995), the vector autoregressive (VAR) model can be represented in terms of the vector error correction model (VECM) as follows:

VAR:

$$
\begin{array}{r}
X_{t}=A_{1} X_{t-1}+A_{2} X_{t-2}+\cdots+A_{1} X_{t-1}+\varepsilon_{t} \\
\Delta \mathbf{X}_{\mathrm{t}}=\Pi \mathbf{X}_{\mathrm{t}-1}+\sum_{\mathrm{i}=1}^{\mathrm{k}-1} \Gamma_{\mathrm{i}} \Delta \mathbf{X}_{\mathrm{t}-1}+\mu+\boldsymbol{\varepsilon}_{\mathrm{t}}
\end{array}
$$


where $\boldsymbol{X}_{t}$ is an endogenous variable which has the dimension $p \times 1$. and $p$ is the number of endogenous variables. $\Pi$ and the $\Gamma_{\mathrm{i}}$ are $p \times p$ matrices of coefficients. $\boldsymbol{\mu}$ is a $p \times$ 1 dimension vector of deterministic variables. $\epsilon$ is $\operatorname{NIID}(0, \Omega)$.

From the construction, the difference of the endogenous variables and their lagged differences are stationary. A test for cointegration can be analyzed in terms of the long run impact matrix, $\Pi$, which can be decomposed as:

$$
\Pi=\boldsymbol{\alpha} \boldsymbol{\beta}^{\prime}
$$

where $\alpha$ and $\beta$ are $p \times r$ matrices of full rank.

Johansen $(1988,1991)$ designs the test for the matrix $\Pi$. The null hypothesis is that rank $(\Pi)=r$, and the alternative hypothesis is that rank $(\Pi)>r$. Johansen and Juselius (1990) use the likelihood ratio test statistic (so called "trace statistic") as follows:

$$
\lambda_{\text {trace }}=-T \sum_{i=r+1}^{p} \ln \left(1-\hat{\lambda}_{l}\right) \quad \sim \chi^{2}(p-r)
$$

where the $\lambda_{\mathrm{i}}$ are the eigenvalues, ordered from smallest to largest. Moreover, the test of determination of cointegration rank is designed for maximum cointegration relations as follows:

$$
\lambda_{\max }=-T \ln \left(1-\widehat{\lambda_{l+1}}\right) \quad \sim \chi^{2}(p-r)
$$

Both tests are distributed asymptotically as chi-square distribution with $p-r$ degree of freedom. 


\subsection{Empirical Results}

3.3.1. Regression Tests of Forward Rate as an Unbiased Predictor of Future Spot Rate

The Unbiased Expectations Hypothesis simply states that forward exchange rate is an unbiased predictor of future spot rate, meaning that the expected future spot rate in the next period $(t=1)$ is equal to the forward exchange rate quoted today for the next period delivery, or mathematically, $E\left(S_{1}\right)=F_{0,1}$. This statement holds when a market is efficient.

In order to test the Unbiased Expectations Hypothesis, we employ equation (1), in which both spot and 90-day forward exchange rates are in terms of the level. However, the level spot and forward rates in equation (1) are nonstationary. The nonstationarity of the time series data causes unconditional expected value and variance to be biased, and the estimated coefficients from the univariate regression equation do not subsequently possess good statistical properties. A possible correction to the problem of nonstationarity is that all variables in the level regression equation have to be converted into first differences. The (percentage) change in the foreign exchange rate is stationary, as presented in equation (2). Under the Unbiased Expectations Hypothesis, the null hypotheses of the estimated intercept and coefficient are equal to 0 and 1 , respectively. If the null hypotheses hold, the foreign exchange market is informationally efficient.

Figure 3 displays the forward premium and change in spot exchange rate of each currency for the entire sample period. The forward premium shows relatively smooth pattern, while the change in spot exchange rate is relatively more volatile than its counterpart forward premium. The stationarity property of these time series allows us to 
examine whether the theory of forward rate as an unbiased predictor of future spot rate is valid or not.

Figure 3 Forward Premium and Spot Exchange Rate Change

Panel A: AUD

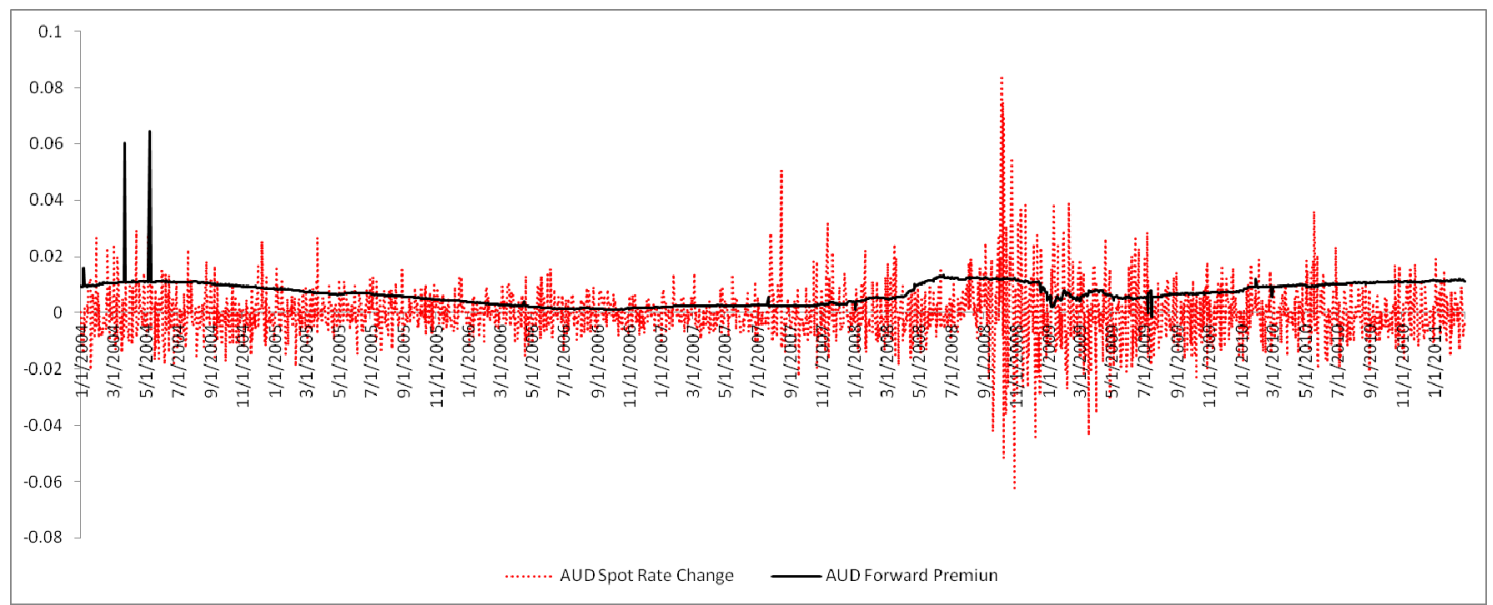

\section{Panel B: CAD}

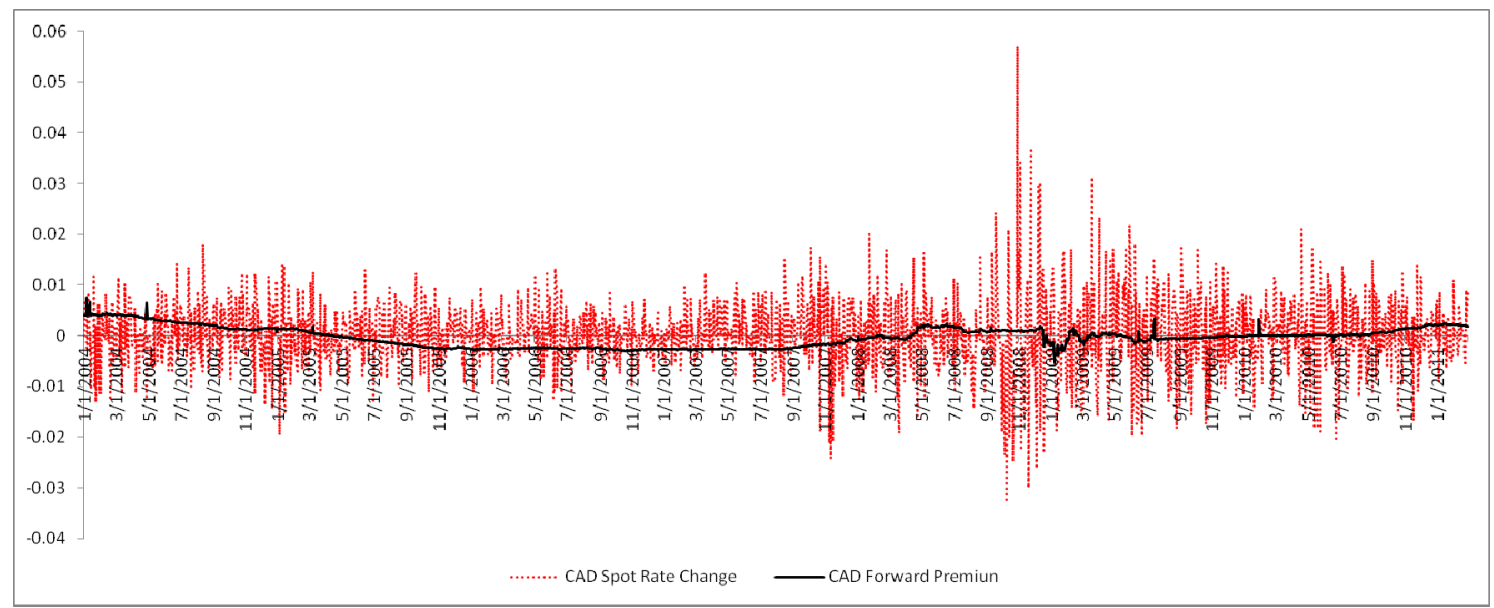




\section{Panel C: CHF}

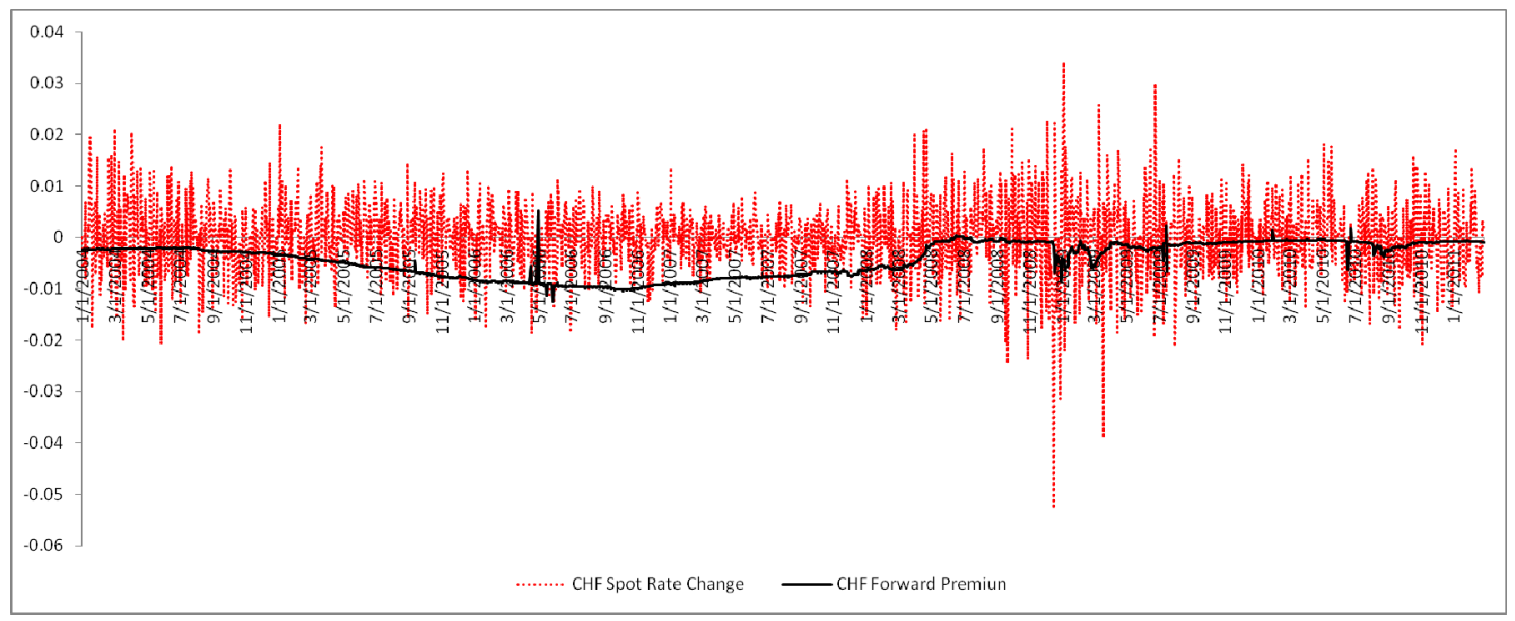

\section{Panel D: EUR}

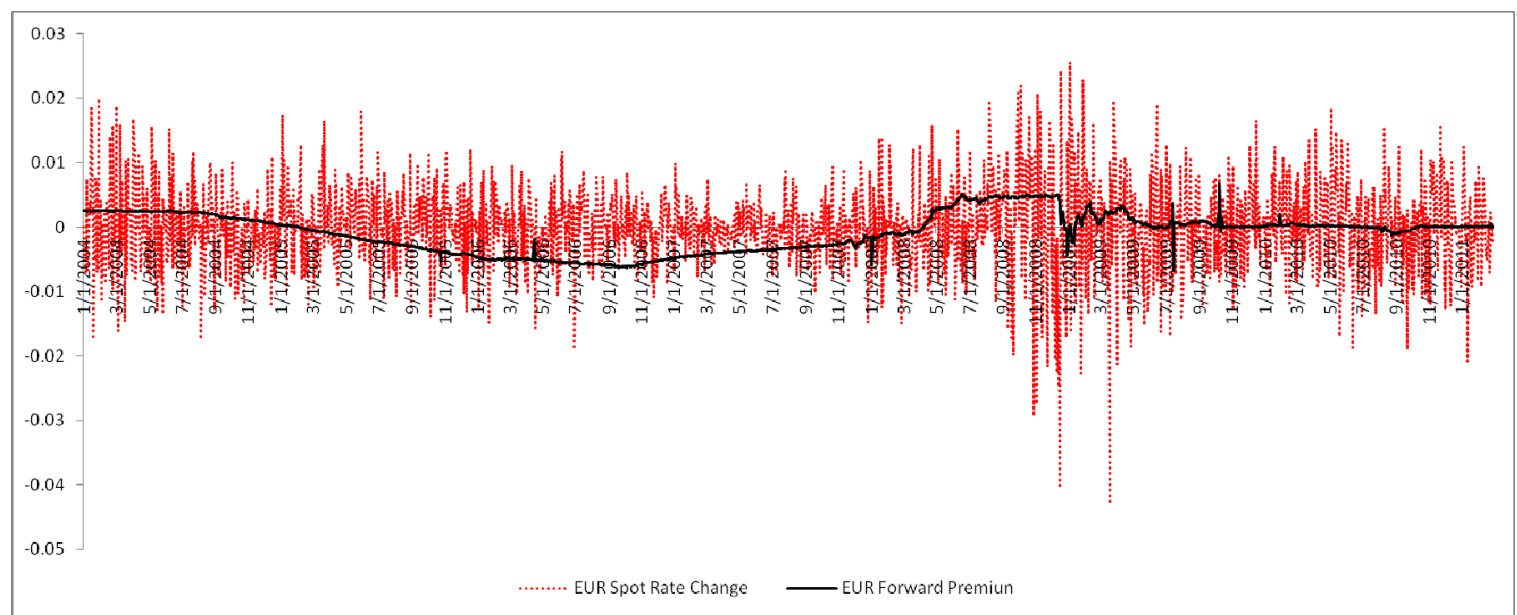




\section{Panel E: GBP}

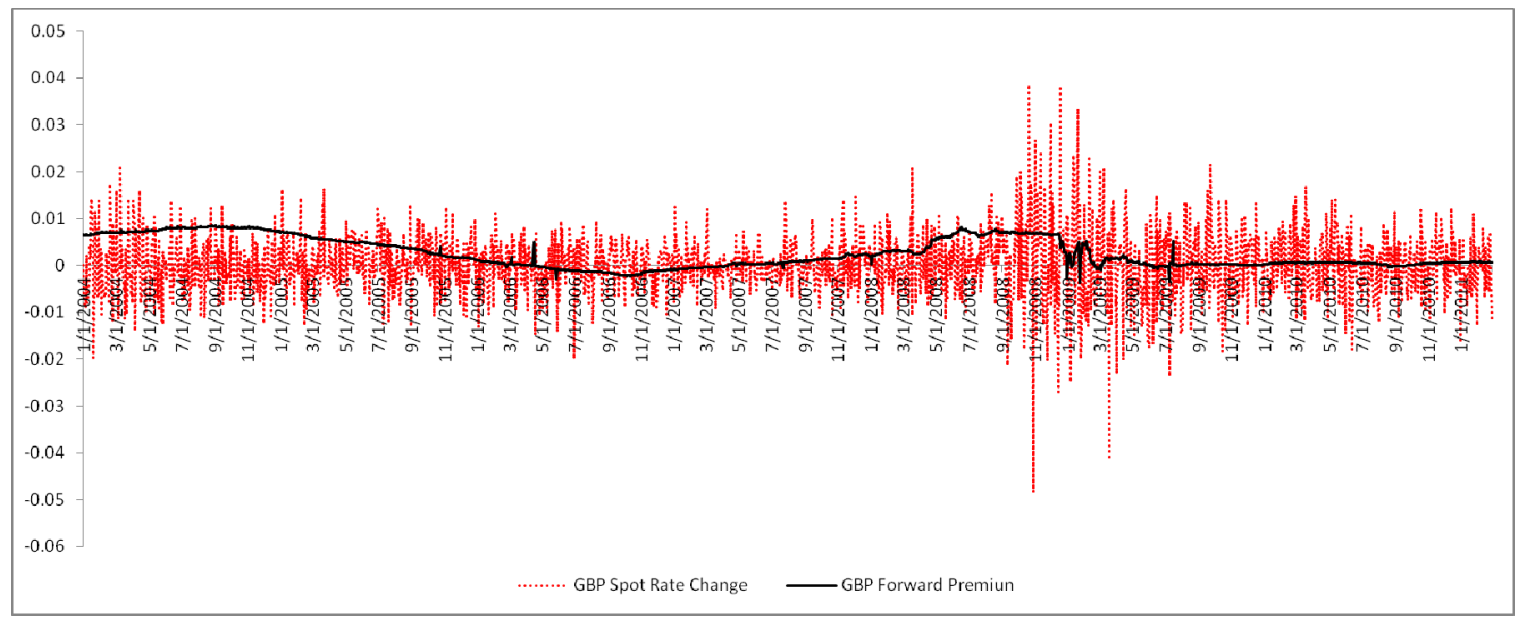

\section{Panel F: JPY}

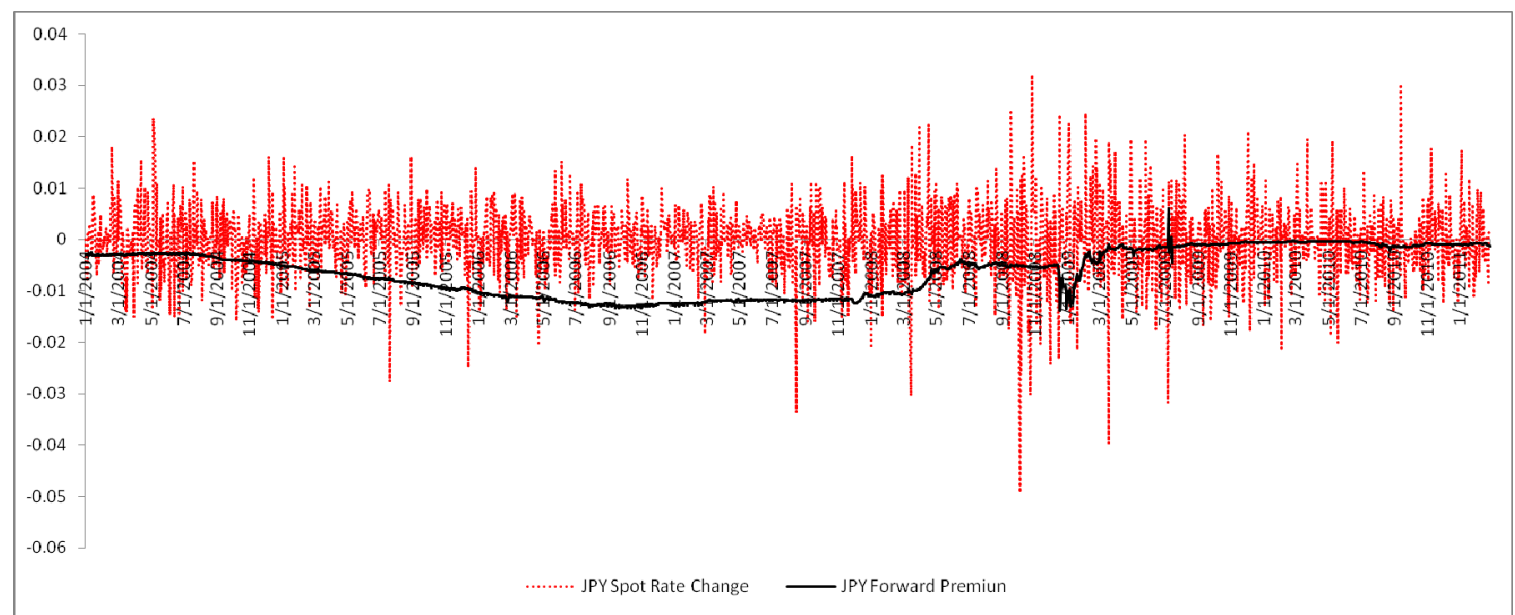

Table 7 presents the estimated intercept and coefficient and the Durbin-Watson statistic obtained from the level regression (as shown in equation (1)) and the change regression (as shown in equation (2)) over the entire sample, non-crisis, and crisis periods, respectively. All estimated coefficients $(\beta)$ for all of the currencies' level regression in equation (1) are positive and significant at the $1 \%$ level, irrespective of the time period. In general, the $t$-statistic values are largest over the entire sample period, and 
are smallest over the crisis period. Most of the estimated intercepts $(\alpha)$ are also significant at the $1 \%$ level. However, the intercept of the JPY over the entire sample period and that of the CAD over the non-crisis period are insignificant, although their corresponding estimated coefficients are significant. The coefficients of determination $\left(\mathrm{R}^{2}\right)$ are relatively high, where they are largest over the entire sample period. For example, the largest $\mathrm{R}^{2}$ is $80.7 \%$ for the JPY and the second best is $73.0 \%$ for the CAD. The $\mathrm{R}^{2}$ values over the non-crisis period are larger than the crisis period, with the exception of the CHF, GBP, and JPY. For the currencies where the coefficients of determination reduce over the crisis period, the values of $\mathrm{R}^{2}$ diminish $50 \%$ of their corresponding values in the non-crisis period, whereas in the other currencies, the $\mathrm{R}^{2}$ values slightly improve in the crisis period.

An analysis of a few additional aspects of our estimation, e.g., Durbin Watson statistic, is in order. The Durbin-Watson statistic determines the problem of autocorrelation. The DW values are too low in the level equations, but acceptable in the change equations (see next analysis). The Durbin-Watson statistic values are significant at the $1 \%$ level for all currencies in all samples. A possible explanation of the significance of the Durbin-Watson statistic is the problem of nonstationarity of the level spot and forward exchange rates employed in the regression model. In sum, the foreign exchange market is not informationally efficient for the AUD, CAD, CHF, EUR, GBP, and JPY in all sample periods, or the forward rate is a poor and biased predictor of the future spot rate in the foreign exchange market. 
Table 7 Univariate Tests of Forward Rate as an Unbiased Predictor of Future Spot Rate

The univariate regression for testing the theory of the 90-day forward rate as an unbiased predictor of future spot rate is below:

$$
\begin{aligned}
& \text { Level: } \quad S_{i, t+90}=\alpha_{i}+\beta_{i} F_{i, t}^{90}+\varepsilon_{i, t} \\
& \text { Change: } \quad s_{i, t}=\delta_{i}+\theta_{i} f_{i, t}+\varepsilon_{i, t}
\end{aligned}
$$

where $s_{i, t}=\operatorname{Ln}\left(S_{i, t}\right)-\operatorname{Ln}\left(S_{i, t-1}\right)$ and $f_{i, t}=\operatorname{Ln}\left(F_{i, t}^{90}\right)-\operatorname{Ln}\left(S_{i, t}\right) . S_{i, t}$ and $F_{i, t}^{90}$ are spot and 90-day forward rates. $i$ refers to the Australian dollar (AUD), Canadian dollar (CAD), Swiss franc (CHF), Euro (EUR), British pound (GBP), and Japanese yen (JPY), respectively. Ln denotes natural logarithms. DW is the Durbin-Watson statistic test for autocorrelation. The $t$-statistics are shown in parentheses and $*, * *$, and

\begin{tabular}{|c|c|c|c|c|c|c|c|c|}
\hline \multirow{2}{*}{ Currency } & \multicolumn{4}{|c|}{ Level } & \multicolumn{4}{|c|}{ Change } \\
\hline & $\alpha$ & $\beta$ & $\mathrm{R}^{2}$ & DW & $\delta$ & $\theta$ & $\mathrm{R}^{2}$ & DW \\
\hline \multicolumn{9}{|c|}{ Panel A: Full Period ( Jan 2004-Feb 2011) } \\
\hline AUD & $\begin{array}{c}0.309 * * * \\
(14.16)\end{array}$ & $\begin{array}{c}0.738 * * * \\
(43.11)\end{array}$ & 0.499 & $0.027 * * *$ & $\begin{array}{c}-0.090^{* *} \\
(-2.03)\end{array}$ & $\begin{array}{l}0.107^{*} \\
(1.92)\end{array}$ & 0.002 & 2.076 \\
\hline CAD & $\begin{array}{c}0.177 * * * \\
(13.12)\end{array}$ & $\begin{array}{c}0.835^{* * *} \\
(71.04)\end{array}$ & 0.730 & $0.035 * * *$ & $\begin{array}{l}0.014 \\
(0.89)\end{array}$ & $\begin{array}{l}-0.040 \\
(-0.49)\end{array}$ & 0.000 & 2.000 \\
\hline $\mathrm{CHF}$ & $\begin{array}{c}0.111 * * * \\
(7.02)\end{array}$ & $\begin{array}{c}0.899 * * * \\
(66.30)\end{array}$ & 0.702 & $0.040 * * *$ & $\begin{array}{l}-0.010 \\
(-0.39)\end{array}$ & $\begin{array}{l}0.012 \\
(0.25)\end{array}$ & 0.000 & 2.103 \\
\hline EUR & $\begin{array}{c}0.204 * * * \\
(17.73)\end{array}$ & $\begin{array}{c}0.728 * * * \\
(48.08)\end{array}$ & 0.553 & $0.027 * * *$ & $\begin{array}{l}-0.000 \\
(-0.02)\end{array}$ & $\begin{array}{l}0.060 \\
(1.18)\end{array}$ & 0.001 & 1.972 \\
\hline GBP & $\begin{array}{c}0.090 * * * \\
(11.50)\end{array}$ & $\begin{array}{c}0.843 * * * \\
(61.81)\end{array}$ & 0.672 & $0.025 * * *$ & $\begin{array}{l}-0.018 \\
(-0.90)\end{array}$ & $\begin{array}{l}0.090 \\
(1.82)\end{array}$ & 0.002 & 1.947 \\
\hline JPY & $\begin{array}{l}-0.920 \\
(-0.77)\end{array}$ & $\begin{array}{c}1.007 * * * \\
(88.45)\end{array}$ & 0.807 & $0.039 * * *$ & $\begin{array}{l}-0.021 \\
(-0.76)\end{array}$ & $\begin{array}{l}-0.010 \\
(-0.28)\end{array}$ & 0.000 & 2.090 \\
\hline
\end{tabular}
$* * *$ are significant at the $10 \%, 5 \%$, and $1 \%$, respectively. 
Table 7 (Continued)

\begin{tabular}{|c|c|c|c|c|c|c|c|c|}
\hline \multirow[t]{2}{*}{ Currency } & \multicolumn{4}{|c|}{ Level } & \multicolumn{4}{|c|}{ Change } \\
\hline & $\alpha$ & $\beta$ & $\mathrm{R}^{2}$ & DW & $\delta$ & $\theta$ & $\mathrm{R}^{2}$ & DW \\
\hline \multicolumn{9}{|c|}{ Panel B: Non-crisis Period (Jan 2004-Dec 2007) } \\
\hline AUD & $\begin{array}{c}0.248 * * * \\
(7.69)\end{array}$ & $\begin{array}{c}0.794 * * * \\
(32.59)\end{array}$ & 0.505 & $0.045 * * *$ & $\begin{array}{c}-0.063 * \\
(-1.83)\end{array}$ & $\begin{array}{c}0.090^{*} \\
(1.77)\end{array}$ & 0.003 & 1.940 \\
\hline CAD & $\begin{array}{l}0.023 \\
(1.23)\end{array}$ & $\begin{array}{c}0.964 * * * \\
(63.25)\end{array}$ & 0.794 & $0.039 * * *$ & $\begin{array}{c}0.021 \\
(1.24)\end{array}$ & $\begin{array}{l}-0.060 \\
(-0.85)\end{array}$ & 0.001 & 2.093 \\
\hline $\mathrm{CHF}$ & $\begin{array}{c}0.703 * * * \\
(18.81)\end{array}$ & $\begin{array}{c}0.430 * * * \\
(14.24)\end{array}$ & 0.163 & $0.037 * * *$ & $\begin{array}{l}-0.003 \\
(-0.07)\end{array}$ & $\begin{array}{l}0.009 \\
(0.14)\end{array}$ & 0.000 & 2.088 \\
\hline EUR & $\begin{array}{c}0.169 * * * \\
(8.32)\end{array}$ & $\begin{array}{c}0.776^{* * *} \\
(30.22)\end{array}$ & 0.467 & $0.032 * * *$ & $\begin{array}{l}-0.007 \\
(-0.36)\end{array}$ & $\begin{array}{l}0.031 \\
(0.54)\end{array}$ & 0.000 & 2.047 \\
\hline GBP & $\begin{array}{c}0.153 * * * \\
(13.44)\end{array}$ & $\begin{array}{c}0.704 * * * \\
(33.63)\end{array}$ & 0.521 & $0.038 * * *$ & $\begin{array}{l}-0.017 \\
(-0.81)\end{array}$ & $\begin{array}{l}0.024 \\
(0.50)\end{array}$ & 0.000 & 2.003 \\
\hline JPY & $\begin{array}{c}29.193 * * * \\
(11.33)\end{array}$ & $\begin{array}{c}0.750 \text { *** } \\
(32.60)\end{array}$ & 0.505 & $0.045 * * *$ & $\begin{array}{l}0.007 \\
(0.16)\end{array}$ & $\begin{array}{l}0.004 \\
(0.09)\end{array}$ & 0.000 & 2.038 \\
\hline \multicolumn{9}{|c|}{ Panel C: Crisis Period (Jan 2008-Feb 2011) } \\
\hline AUD & $\begin{array}{c}0.407 * * * \\
(11.57)\end{array}$ & $\begin{array}{c}0.644 * * * \\
(22.11)\end{array}$ & 0.373 & $0.023 * * *$ & $\begin{array}{c}-0.253^{*} \\
(-1.73)\end{array}$ & $\begin{array}{c}0.267 * \\
(1.67)\end{array}$ & 0.003 & 2.136 \\
\hline CAD & $\begin{array}{c}0.374 * * * \\
(13.43)\end{array}$ & $\begin{array}{c}0.653 * * * \\
(25.27)\end{array}$ & 0.437 & $0.033 * * *$ & $\begin{array}{l}-0.004 \\
(-0.13)\end{array}$ & $\begin{array}{l}0.189 \\
(0.67)\end{array}$ & 0.001 & 1.954 \\
\hline $\mathrm{CHF}$ & $\begin{array}{c}0.517 * * * \\
(14.12)\end{array}$ & $\begin{array}{c}0.508 * * * \\
(14.99)\end{array}$ & 0.215 & $0.031 * * *$ & $\begin{array}{l}0.011 \\
(0.28)\end{array}$ & $\begin{array}{l}0.209 \\
(1.25)\end{array}$ & 0.002 & 2.119 \\
\hline EUR & $\begin{array}{c}0.338 * * * \\
(15.64)\end{array}$ & $\begin{array}{c}0.533 * * * \\
(17.71)\end{array}$ & 0.276 & $0.023 * * *$ & $\begin{array}{l}-0.007 \\
(-0.24)\end{array}$ & $\begin{array}{l}0.133 \\
(0.94)\end{array}$ & 0.001 & 1.928 \\
\hline GBP & $\begin{array}{c}0.204 * * * \\
(16.31)\end{array}$ & $\begin{array}{c}0.678 * * * \\
(32.82)\end{array}$ & 0.567 & $0.024 * * *$ & $\begin{array}{l}-0.003 \\
(-0.85)\end{array}$ & $\begin{array}{c}0.264 * * \\
(2.44)\end{array}$ & 0.007 & 1.928 \\
\hline JPY & $\begin{array}{c}24.419 * * * \\
(11.62)\end{array}$ & $\begin{array}{c}0.735 * * * \\
(35.31)\end{array}$ & 0.602 & $0.036^{* * *}$ & $\begin{array}{l}-0.015 \\
(-0.38)\end{array}$ & $\begin{array}{l}0.072 \\
(0.83)\end{array}$ & 0.001 & 2.123 \\
\hline
\end{tabular}


Given the problem of nonstationarity, the regression model in terms of change in variables is introduced as presented in equation (2). The results of the change regression model are opposite to those of the level regression model. The estimated intercepts $(\delta)$ and coefficients $(\theta)$ are insignificant, with the exception of the AUD and GBP. Most of the coefficients $(\theta)$ are positive and insignificant. Both estimated intercept and coefficient of the AUD in all periods are significant at least at the $10 \%$ level, whereas the estimated coefficient of the GBP is significant at the 5\% level over the crisis period. However, the $\mathrm{R}^{2}$ values are diminished extensively compared with the regression of the level foreign exchange rate. The largest $\mathrm{R}^{2}$ value $(0.7 \%)$ is from the GBP over the crisis period. Most of the $\mathrm{R}^{2}$ value is from $0.0 \%$ to $0.3 \%$. Obviously, the problem of nonstationarity is mitigated; the Durbin-Watson statistic values are increased though they are not statistically significant in any period and in any currencies.

In sum, the foreign exchange market results from the change in the variables regression model is informationally inefficient, irrespective of the time period considered. In statistical terms, the forward rate does not seem to be unbiased predictor of the future spot rate in the foreign exchange market. The hypotheses of zero value for the intercept and value of one for the coefficient are not statistically supported.

\subsubsection{GARCH $(1,1)$ Specification Model}

This analysis introduces the GARCH $(1,1)$ model for the Unbiased Expectations Hypothesis. Table 8 shows the results of the estimated parameters employing the GARCH $(1,1)$ model for the change univariate regression model. The GARCH $(1,1)$ model is presented in equation (3). The forward premium over the entire sample and non- 
crisis periods is insignificant, but the sign of the forward premium is random: negative for the CAD and JPY and positive for the remaining. However, the evidence in the crisis period is odd. The forward premium of the CHF, EUR, and GBP is significant at a $10 \%$ level, and that of the AUD, CAD, and JPY is still insignificant. Moreover, the sign of forward premium is positive in all cases over the crisis period. The estimated intercepts are not significant for all cases, except for the CAD over the entire sample period. The unconditional variance $\left(\beta_{0}\right)$ parameter is not strongly significant, especially over the noncrisis period. Thus, the significance of unconditional variance over the entire sample period is affected by the variability during the crisis period. Nevertheless, the parameter estimates of the $\beta_{1}$ and $\beta_{2}$ are strongly significant, though not equal to 0 and 1 , and represent time-varying volatility in the foreign exchange market. ${ }^{1}$

The coefficients of determination $\left(\mathrm{R}^{2}\right)$ from the GARCH $(1,1)$ model are generally less than those of the change univariate regression model, but not significant. The largest $\mathrm{R}^{2}$ value $(0.5 \%)$ from the GARCH $(1,1)$ model is from the JPY over the crisis period. However, we cannot directly compare the $\mathrm{R}^{2}$ from these two methodologies because the underlying assumptions are not the same. In conclusion, foreign exchange markets are still informationally inefficient after considering time-varying characteristics of foreign exchange rates. Arbitrage opportunities thus could exist. The results from the GARCH $(1,1)$ model over the crisis period show that the estimates of the forward premium are significant at the $10 \%$ level for the CHF, EUR, and GBP, demonstrating that the forward premium is not a good estimator of future spot rate.

\footnotetext{
${ }^{1}$ The $\beta_{1}$ and $\beta_{2}$ parameters of the JPY over the crisis period are significant at the $5 \%$ and $10 \%$ levels, respectively.
} 


\section{Table 8 GARCH $(1,1)$ Specification Model}

The GARCH $(1,1)$ specification is given as follows:

Change: $\quad s_{i, t+90}=\delta_{i}+\theta_{i} f_{i, t}+\varepsilon_{i, t}$

where $\varepsilon_{t} \mid \Omega_{t-1} \sim N\left(0, h_{t}\right)$

and $h_{t}=\beta_{0}+\beta_{1} h_{t-1}+\beta_{2} \varepsilon_{t-1}^{2}$ where $\varepsilon_{t}$ is the innovation term, $\mathrm{h}_{\mathrm{t}}$ is the conditional variance, and $\beta_{0}, \beta_{1}$, and $\beta_{2}$ are constant parameters. The $t$-statistics are shown in parentheses and $*{ }^{*}$, , and $* * *$ are significant at the $10 \%, 5 \%$, and $1 \%$, respectively.

\begin{tabular}{|c|c|c|c|c|c|c|}
\hline Currency & Intercept & $\begin{array}{l}\text { Forward } \\
\text { Premium }\end{array}$ & $\beta_{0}$ & $\beta_{1}$ & $\beta_{2}$ & $\overline{\mathrm{R}^{2}}$ \\
\hline \multicolumn{7}{|c|}{ Panel A: Full Period (Jan 2004-Feb 2011) } \\
\hline AUD & $\begin{array}{l}-0.000 \\
(-1.47)\end{array}$ & $\begin{array}{l}0.040 \\
(0.97)\end{array}$ & $\begin{array}{c}0.000 * * * \\
(3.93)\end{array}$ & $\begin{array}{c}0.056^{* * *} \\
(11.60)\end{array}$ & $\begin{array}{c}0.938 * * * \\
(153.29)\end{array}$ & 0.001 \\
\hline CAD & $\begin{array}{l}0.000^{*} \\
(1.76)\end{array}$ & $\begin{array}{l}-0.010 \\
(-0.16)\end{array}$ & $\begin{array}{c}0.000^{* *} \\
(2.50)\end{array}$ & $\begin{array}{c}0.047 * * * \\
(7.78)\end{array}$ & $\begin{array}{c}0.947 * * * \\
(133.50)\end{array}$ & 0.000 \\
\hline $\mathrm{CHF}$ & $\begin{array}{l}-0.000 \\
(-0.37)\end{array}$ & $\begin{array}{l}0.015 \\
(0.34)\end{array}$ & $\begin{array}{c}0.000^{*} \\
(1.76)\end{array}$ & $\begin{array}{c}0.027 * * * \\
(6.20)\end{array}$ & $\begin{array}{c}0.970 * * * \\
(194.52)\end{array}$ & 0.000 \\
\hline EUR & $\begin{array}{l}-0.000 \\
(-0.44)\end{array}$ & $\begin{array}{l}0.058 \\
(1.39)\end{array}$ & $\begin{array}{c}0.000 * \\
(1.72)\end{array}$ & $\begin{array}{c}0.031 * * * \\
(6.63)\end{array}$ & $\begin{array}{c}0.968 * * * \\
(219.92)\end{array}$ & 0.001 \\
\hline GBP & $\begin{array}{l}-0.000 \\
(-1.17)\end{array}$ & $\begin{array}{l}0.059 \\
(1.45)\end{array}$ & $\begin{array}{c}0.000^{* *} \\
(2.44)\end{array}$ & $\begin{array}{c}0.034 * * * \\
(7.38)\end{array}$ & $\begin{array}{c}0.963 * * * \\
(177.62)\end{array}$ & 0.001 \\
\hline JPY & $\begin{array}{l}-0.000 \\
(-0.78)\end{array}$ & $\begin{array}{l}-0.020 \\
(-0.61)\end{array}$ & $\begin{array}{c}0.000^{* * *} \\
(4.78)\end{array}$ & $\begin{array}{c}0.026^{* * *} \\
(7.13)\end{array}$ & $\begin{array}{c}0.966 * * * \\
(215.41)\end{array}$ & 0.000 \\
\hline \multicolumn{7}{|c|}{ Panel B: Non-Crisis Period (Jan 2004-Dec 2007) } \\
\hline AUD & $\begin{array}{l}-0.001 \\
(-1.33)\end{array}$ & $\begin{array}{l}0.030 \\
(0.52)\end{array}$ & $\begin{array}{c}0.000 * * * \\
(6.14)\end{array}$ & $\begin{array}{c}0.216^{* * *} \\
(8.74)\end{array}$ & $\begin{array}{l}0.145 \\
(1.26)\end{array}$ & 0.000 \\
\hline CAD & $\begin{array}{l}0.000 \\
(1.15)\end{array}$ & $\begin{array}{l}-0.037 \\
(-0.55)\end{array}$ & $\begin{array}{l}0.000 \\
(1.43)\end{array}$ & $\begin{array}{c}0.039 * * * \\
(3.66)\end{array}$ & $\begin{array}{c}0.949 * * * \\
(54.00)\end{array}$ & 0.001 \\
\hline $\mathrm{CHF}$ & $\begin{array}{l}-0.000 \\
(-0.08)\end{array}$ & $\begin{array}{l}0.014 \\
(0.20)\end{array}$ & $\begin{array}{l}0.000 \\
(1.17)\end{array}$ & $\begin{array}{c}0.020 * * * \\
(3.07)\end{array}$ & $\begin{array}{c}0.978 * * * \\
(135.88)\end{array}$ & 0.000 \\
\hline EUR & $\begin{array}{l}-0.000 \\
(-0.65)\end{array}$ & $\begin{array}{l}0.031 \\
(0.52)\end{array}$ & $\begin{array}{l}0.000 \\
(1.22)\end{array}$ & $\begin{array}{c}0.026^{* * *} \\
(3.72)\end{array}$ & $\begin{array}{c}0.972 * * * \\
(132.55)\end{array}$ & 0.000 \\
\hline GBP & $\begin{array}{l}-0.000 \\
(-1.02)\end{array}$ & $\begin{array}{l}0.038 \\
(0.77)\end{array}$ & $\begin{array}{c}0.000 \\
(-1.48)\end{array}$ & $\begin{array}{c}0.023 * * * \\
(3.20)\end{array}$ & $\begin{array}{c}0.971 * * * \\
(99.99)\end{array}$ & 0.000 \\
\hline JPY & $\begin{array}{l}-0.000 \\
(-0.02)\end{array}$ & $\begin{array}{l}-0.009 \\
(-0.19)\end{array}$ & $\begin{array}{c}0.000^{* *} \\
(2.54)\end{array}$ & $\begin{array}{c}0.029 * * * \\
(3.19)\end{array}$ & $\begin{array}{c}0.938^{* * *} \\
(47.62)\end{array}$ & 0.000 \\
\hline \multicolumn{7}{|c|}{ Panel C: Crisis Period (Jan 2008-Feb 2011) } \\
\hline AUD & $\begin{array}{l}-0.002 \\
(-1.62)\end{array}$ & $\begin{array}{l}0.166 \\
(1.27)\end{array}$ & $\begin{array}{c}0.000^{* *} \\
(2.44)\end{array}$ & $\begin{array}{c}0.082 * * * \\
(7.04)\end{array}$ & $\begin{array}{c}0.906 * * * \\
(69.16)\end{array}$ & 0.002 \\
\hline CAD & $\begin{array}{l}0.000 \\
(0.45)\end{array}$ & $\begin{array}{l}0.196 \\
(0.77)\end{array}$ & $\begin{array}{l}0.000^{*} \\
(1.91)\end{array}$ & $\begin{array}{c}0.057 * * * \\
(5.34)\end{array}$ & $\begin{array}{c}0.932 * * * \\
(69.96)\end{array}$ & 0.000 \\
\hline $\mathrm{CHF}$ & $\begin{array}{l}0.000 \\
(0.61)\end{array}$ & $\begin{array}{c}0.310^{*} \\
(1.93)\end{array}$ & $\begin{array}{c}0.000^{*} \\
(1.92)\end{array}$ & $\begin{array}{c}0.035 * * * \\
(4.84)\end{array}$ & $\begin{array}{c}0.952 * * * \\
(86.78)\end{array}$ & 0.001 \\
\hline EUR & $\begin{array}{l}-0.000 \\
(-0.73)\end{array}$ & $\begin{array}{l}0.229 * \\
(1.90)\end{array}$ & $\begin{array}{c}0.000^{* *} \\
(2.25)\end{array}$ & $\begin{array}{c}0.032 * * * \\
(4.77)\end{array}$ & $\begin{array}{c}0.959 * * * \\
(146.51)\end{array}$ & 0.001 \\
\hline GBP & $\begin{array}{l}-0.000 \\
(-0.76)\end{array}$ & $\begin{array}{l}0.146^{*} \\
(1.85)\end{array}$ & $\begin{array}{c}0.000^{*} \\
(1.88)\end{array}$ & $\begin{array}{c}0.046^{* * *} \\
(5.53)\end{array}$ & $\begin{array}{c}0.947 * * * \\
(101.06)\end{array}$ & 0.005 \\
\hline JPY & $\begin{array}{l}-0.000 \\
(-0.65)\end{array}$ & $\begin{array}{l}0.037 \\
(0.42)\end{array}$ & $\begin{array}{c}0.000 * * * \\
(3.61)\end{array}$ & $\begin{array}{c}0.114^{* *} \\
(2.50)\end{array}$ & $\begin{array}{l}0.339^{*} \\
(1.88)\end{array}$ & 0.001 \\
\hline
\end{tabular}


3.3.3. Tests of Equality of Estimated Intercepts and Coefficients Between the Non-Crisis and Crisis Periods of the GARCH $(1,1)$ Model

We further analyze the GARCH $(1,1)$ models by comparing the equality of the estimated parameters between the non-crisis and crisis periods. Table 9 presents the statistical test results for such comparison. The equation (4) is employed to test the equality of these parameters. First, the equality of the estimated intercepts and coefficients independently is tested. Then, we perform the joint test equality of these two parameters. The results show that the difference of estimated intercepts is not statistically significant for all currencies and approaches zero. In addition, there is no statistical difference in the estimated coefficients. However, it is important to note that the difference in estimated coefficients of the CHF is statistically significant between the two periods. This result points to the different behavior of the markets within the efficiency tests.

Importantly, the independent tests of the difference in estimated intercepts and coefficients are not sufficient to test whether or not the models over the non-crisis and crisis periods are the same. What matters in this analysis is whether the relation between the spot and its corresponding forward rates remains the same over the non-crisis and crisis periods. The Chow test (joint test) shows that there is no structural break over the sample periods, except for CHF. The GARCH $(1,1)$ models for the CHF are statistically and significantly different between these two periods, or there is a structural shift. 
Table 9 Tests of Equality of Estimated Intercepts and Coefficients Between the Non-Crisis and Crisis Periods of the GARCH $(1,1)$ Model

The test between the intercepts and coefficients of the GARCH $(1,1)$ model over the non-crisis and crisis periods are presented. The F-Value is a statistic test for joint assumption (Chow test). The $t$-statistics are shown in parentheses and $*, * *$, and $* * *$ are significant at the $10 \%, 5 \%$, and $1 \%$, respectively.

\begin{tabular}{cccc}
\hline Currency & Intercept & Coefficient & F-Value \\
\hline AUD & 0.001 & -0.084 & 1.90 \\
& $(1.28)$ & $(-0.65)$ & \\
CAD & 0.000 & -0.242 & 0.47 \\
& $(0.26)$ & $(-0.97)$ & \\
CHF & -0.000 & $-0.293^{* *}$ & $3.06^{* *}$ \\
& $(-0.41)$ & $(-1.99)$ & \\
EUR & 0.000 & -0.191 & 1.18 \\
& $(0.13)$ & $(-1.54)$ & \\
GBP & 0.000 & -0.103 & 1.20 \\
& $(-0.01)$ & $(-1.17)$ & 1.26 \\
JPY & 0.000 & -0.071 & \\
& $(0.31)$ & $(-0.79)$ & \\
\hline
\end{tabular}

\subsubsection{Bivariate Cointegration Analysis}

The first step before performing cointegration analysis is to verify whether the time series data are stationary or not. The unit root or non-stationarity test is required. Panels A, B, and C of Table 10 report the results of the Augmented Dickey-Fuller (ADF) and Phillips-Perrons (PP) tests for all spot and forward exchange rates, respectively. Neither ADF nor PP tests for each of the sample periods can reject the null hypothesis of a unit root or nonstationarity test in levels for all exchange rates. These results are consistent with previous findings, showing that the foreign exchange rates possess a unit root in levels. However, the tests of the first difference in level exchange rates show statistically significant, rejecting the null hypothesis of nonstationarity. ${ }^{2}$ In conclusion,

\footnotetext{
${ }^{2}$ An important issue on first difference variable is that it does not carry full long-run information (See Aroskar et al. (2004)).
} 
the first differences of the spot and forward rate are stationary; this is represented as integrated of order zero $(I(0))$. The stationarity of the first difference in exchange provides a good start for the cointegration analysis.

Table 10 Stationarity Tests of Spot and Forward Exchange Rates

This table presents the statistics of the stationary tests of the spot and forward exchange. The Augmented Dickey-Fuller (ADF) and the Phillips-Perron (PP) test is for the null hypothesis of unit root test in data. The $p$-values are shown in parentheses. The optimal lag length is determined by the Akaike Information Criterion (AIC). $* * *$ is significant at the $1 \%$ level.

Panel A: Entire Sample Period

\begin{tabular}{|c|c|c|c|c|c|c|}
\hline \multirow[t]{2}{*}{ Currency } & \multicolumn{3}{|c|}{ Level } & \multicolumn{3}{|c|}{ First Difference } \\
\hline & Lags & $\mathrm{ADF}$ & PP & Lags & $\mathrm{ADF}$ & PP \\
\hline \multicolumn{7}{|l|}{ Spot Rate } \\
\hline AUD & 1 & $\begin{array}{c}-1.49 \\
(0.539)\end{array}$ & $\begin{array}{c}-1.55 \\
(0.507)\end{array}$ & 0 & $\begin{array}{c}-44.99 * * * \\
(0.000)\end{array}$ & $\begin{array}{c}-44.99 * * * \\
(0.000)\end{array}$ \\
\hline CAD & 1 & $\begin{array}{c}-1.58 \\
(0.493)\end{array}$ & $\begin{array}{c}0.418 \\
(-1.620)\end{array}$ & 0 & $\begin{array}{c}-43.15^{* * *} \\
(0.000)\end{array}$ & $\begin{array}{c}-43.15^{* * * *} \\
(0.000)\end{array}$ \\
\hline $\mathrm{CHF}$ & 1 & $\begin{array}{c}-0.93 \\
(0.779)\end{array}$ & $\begin{array}{l}-1.01 \\
(0.752)\end{array}$ & 0 & $\begin{array}{c}-45.50 * * * \\
(0.000)\end{array}$ & $\begin{array}{c}-45.50 * * * \\
(0.000)\end{array}$ \\
\hline EUR & 1 & $\begin{array}{c}-1.96 \\
(0.305)\end{array}$ & $\begin{array}{c}-1.96 \\
(0.305)\end{array}$ & 0 & $\begin{array}{c}-42.57 * * * \\
(0.000)\end{array}$ & $\begin{array}{c}-42.57 * * * \\
(0.000)\end{array}$ \\
\hline GBP & 1 & $\begin{array}{l}-1.99 \\
(0.607)\end{array}$ & $\begin{array}{l}-1.40 \\
(0.583)\end{array}$ & 0 & $\begin{array}{c}-41.67 * * * \\
(0.000)\end{array}$ & $\begin{array}{c}-41.67 * * * \\
(0.000)\end{array}$ \\
\hline JPY & 1 & $\begin{array}{c}-2.01 \\
(0.598)\end{array}$ & $\begin{array}{c}-0.54 \\
(0.881)\end{array}$ & 0 & $\begin{array}{c}-45.11 * * * \\
(0.000)\end{array}$ & $\begin{array}{c}-45.11 * * * \\
(0.000)\end{array}$ \\
\hline \multicolumn{7}{|c|}{ Forward Rate } \\
\hline AUD & 1 & $\begin{array}{c}-1.52 \\
(0.524)\end{array}$ & $\begin{array}{c}-1.61 \\
(0.479)\end{array}$ & 0 & $\begin{array}{c}-45.79 * * * \\
(0.000)\end{array}$ & $\begin{array}{c}-45.79 * * * \\
(0.000)\end{array}$ \\
\hline CAD & 1 & $\begin{array}{l}-1.60 \\
(0.482)\end{array}$ & $\begin{array}{l}-1.64 \\
(0.462)\end{array}$ & 0 & $\begin{array}{c}-43.18^{* * * *} \\
(0.000)\end{array}$ & $\begin{array}{c}-43.18^{* * * *} \\
(0.000)\end{array}$ \\
\hline $\mathrm{CHF}$ & 1 & $\begin{array}{l}-1.00 \\
(0.757)\end{array}$ & $\begin{array}{l}-1.08 \\
(0.727)\end{array}$ & 0 & $\begin{array}{c}-45.54 * * * \\
(0.000)\end{array}$ & $\begin{array}{c}-45.54 * * * \\
(0.000)\end{array}$ \\
\hline EUR & 1 & $\begin{array}{c}-2.01 \\
(0.281)\end{array}$ & $\begin{array}{c}-2.01 \\
(0.282)\end{array}$ & 0 & $\begin{array}{c}-42.36^{* * *} \\
(0.000)\end{array}$ & $\begin{array}{c}-42.36^{* * * *} \\
(0.000)\end{array}$ \\
\hline GBP & 1 & $\begin{array}{c}-1.43 \\
(0.568)\end{array}$ & $\begin{array}{l}-1.41 \\
(0.580)\end{array}$ & 0 & $\begin{array}{c}-42.05^{* * * *} \\
(0.000)\end{array}$ & $\begin{array}{c}-42.05 * * * \\
(0.000)\end{array}$ \\
\hline JPY & 1 & $\begin{array}{c}-0.55 \\
(0.879)\end{array}$ & $\begin{array}{c}-0.60 \\
(0.868)\end{array}$ & 0 & $\begin{array}{c}-45.06 * * * \\
(0.000)\end{array}$ & $\begin{array}{c}-45.06 * * * \\
(0.000)\end{array}$ \\
\hline
\end{tabular}


Panel B: Non-Crisis Period

\begin{tabular}{|c|c|c|c|c|c|c|}
\hline \multirow[t]{2}{*}{ Currency } & \multicolumn{3}{|c|}{ Level } & \multicolumn{3}{|c|}{ First Difference } \\
\hline & Lags & $\mathrm{ADF}$ & PP & Lags & $\mathrm{ADF}$ & $\mathrm{PP}$ \\
\hline \multicolumn{7}{|l|}{ Spot Rate } \\
\hline AUD & 1 & $\begin{array}{c}-1.41 \\
(0.579)\end{array}$ & $\begin{array}{c}-1.40 \\
(0.585)\end{array}$ & 0 & $\begin{array}{c}-30.67 * * * \\
(0.000)\end{array}$ & $\begin{array}{c}-30.67 * * * \\
(0.000)\end{array}$ \\
\hline CAD & 2 & $\begin{array}{c}-0.27 \\
(0.926)\end{array}$ & $\begin{array}{c}-0.40 \\
(0.907)\end{array}$ & 1 & $\begin{array}{c}-23.26 * * * \\
(0.000)\end{array}$ & $\begin{array}{c}-33.82 * * * \\
(0.000)\end{array}$ \\
\hline $\mathrm{CHF}$ & 1 & $\begin{array}{c}-1.97 \\
(0.300)\end{array}$ & $\begin{array}{l}-2.05 \\
(0.266)\end{array}$ & 0 & $\begin{array}{c}-33.79 * * * \\
(0.000)\end{array}$ & $\begin{array}{c}-33.79 * * * \\
(0.000)\end{array}$ \\
\hline EUR & 1 & $\begin{array}{c}-0.70 \\
(0.846)\end{array}$ & $\begin{array}{c}-0.74 \\
(0.834)\end{array}$ & 0 & $\begin{array}{c}-33.14 * * * \\
(0.000)\end{array}$ & $\begin{array}{c}-33.14 * * * \\
(0.000)\end{array}$ \\
\hline GBP & 1 & $\begin{array}{c}-1.67 \\
(0.445)\end{array}$ & $\begin{array}{c}-1.73 \\
(0.418)\end{array}$ & 0 & $\begin{array}{c}-32.42 * * * \\
(0.000)\end{array}$ & $\begin{array}{c}-32.42 * * * \\
(0.000)\end{array}$ \\
\hline JPY & 1 & $\begin{array}{c}-2.05 \\
(0.266)\end{array}$ & $\begin{array}{c}-2.03 \\
(0.274)\end{array}$ & 0 & $\begin{array}{c}-32.78 * * * \\
(0.000)\end{array}$ & $\begin{array}{c}-32.78 * * * \\
(0.000)\end{array}$ \\
\hline \multicolumn{7}{|c|}{ Forward Rate } \\
\hline AUD & 1 & $\begin{array}{c}-1.42 \\
(0.576)\end{array}$ & $\begin{array}{c}-1.47 \\
(0.550)\end{array}$ & 0 & $\begin{array}{c}-30.57 * * * \\
(0.000)\end{array}$ & $\begin{array}{c}-30.57 * * * \\
(0.000)\end{array}$ \\
\hline CAD & 2 & $\begin{array}{c}-0.31 \\
(0.921)\end{array}$ & $\begin{array}{c}-0.43 \\
(0.901)\end{array}$ & 1 & $\begin{array}{c}-23.17 * * * \\
(0.000)\end{array}$ & $\begin{array}{c}-33.81 * * * \\
(0.000)\end{array}$ \\
\hline $\mathrm{CHF}$ & 1 & $\begin{array}{c}-2.02 \\
(0.277)\end{array}$ & $\begin{array}{c}-2.11 \\
(0.241)\end{array}$ & 0 & $\begin{array}{c}-33.88 * * * \\
(0.000)\end{array}$ & $\begin{array}{c}-33.88 * * * \\
(0.000)\end{array}$ \\
\hline EUR & 1 & $\begin{array}{c}-0.72 \\
(0.839)\end{array}$ & $\begin{array}{c}-0.77 \\
(0.826)\end{array}$ & 0 & $\begin{array}{c}-33.18 * * * \\
(0.000)\end{array}$ & $\begin{array}{c}-33.18 * * * \\
(0.000)\end{array}$ \\
\hline GBP & 1 & $\begin{array}{c}-1.68 \\
(0.441)\end{array}$ & $\begin{array}{c}-1.74 \\
(0.412)\end{array}$ & 0 & $\begin{array}{c}-32.43 * * * \\
(0.000)\end{array}$ & $\begin{array}{c}-32.43 * * * \\
(0.000)\end{array}$ \\
\hline JPY & 1 & $\begin{array}{c}-2.12 \\
(0.238) \\
\end{array}$ & $\begin{array}{c}-2.10 \\
(0.243) \\
\end{array}$ & 0 & $\begin{array}{c}-32.81 * * * \\
(0.000)\end{array}$ & $\begin{array}{c}-32.81 * * * \\
(0.000) \\
\end{array}$ \\
\hline
\end{tabular}


Panel C: Crisis Period

\begin{tabular}{|c|c|c|c|c|c|c|}
\hline \multirow[t]{2}{*}{ Currency } & \multicolumn{3}{|c|}{ Level } & \multicolumn{3}{|c|}{ First Difference } \\
\hline & Lags & $\mathrm{ADF}$ & PP & Lags & $\mathrm{ADF}$ & PP \\
\hline \multicolumn{7}{|l|}{ Spot Rate } \\
\hline AUD & 1 & $\begin{array}{c}-1.07 \\
(0.728)\end{array}$ & $\begin{array}{c}-1.13 \\
(0.708)\end{array}$ & 0 & $\begin{array}{c}-45.11 * * * \\
(0.000)\end{array}$ & $\begin{array}{c}-45.11 * * * \\
(0.000)\end{array}$ \\
\hline CAD & 1 & $\begin{array}{c}-1.61 \\
(0.476)\end{array}$ & $\begin{array}{c}-1.60 \\
(0.483)\end{array}$ & 0 & $\begin{array}{c}-27.91 * * * \\
(0.000)\end{array}$ & $\begin{array}{c}-27.91 * * * \\
(0.000)\end{array}$ \\
\hline $\mathrm{CHF}$ & 1 & $\begin{array}{c}-1.38 \\
(0.594)\end{array}$ & $\begin{array}{c}-1.52 \\
(0.523)\end{array}$ & 0 & $\begin{array}{c}-30.36^{* * * *} \\
(0.000)\end{array}$ & $\begin{array}{c}-30.36^{* * *} \\
(0.000)\end{array}$ \\
\hline EUR & 1 & $\begin{array}{c}-1.82 \\
(0.373)\end{array}$ & $\begin{array}{c}-1.75 \\
(0.405)\end{array}$ & 0 & $\begin{array}{c}-27.46^{* * * *} \\
(0.000)\end{array}$ & $\begin{array}{c}-27.46^{* * * *} \\
(0.000)\end{array}$ \\
\hline GBP & 1 & $\begin{array}{c}-1.86 \\
(0.350)\end{array}$ & $\begin{array}{c}-1.85 \\
(0.356)\end{array}$ & 0 & $\begin{array}{c}-27.25 * * * \\
(0.000)\end{array}$ & $\begin{array}{c}-27.25 * * * \\
(0.000)\end{array}$ \\
\hline JPY & 1 & $\begin{array}{c}-1.54 \\
(0.516)\end{array}$ & $\begin{array}{c}-1.78 \\
(0.393)\end{array}$ & 0 & $\begin{array}{c}-30.67 * * * \\
(0.000)\end{array}$ & $\begin{array}{c}-30.67 * * * \\
(0.000)\end{array}$ \\
\hline \multicolumn{7}{|c|}{ Forward Rate } \\
\hline AUD & 1 & $\begin{array}{c}-1.08 \\
(0.727)\end{array}$ & $\begin{array}{c}-1.13 \\
(0.705)\end{array}$ & 0 & $\begin{array}{c}-45.06^{* * * *} \\
(0.000)\end{array}$ & $\begin{array}{c}-45.06^{* * * *} \\
(0.000)\end{array}$ \\
\hline CAD & 1 & $\begin{array}{c}-1.62 \\
(0.470)\end{array}$ & $\begin{array}{c}-1.61 \\
(0.477)\end{array}$ & 0 & $\begin{array}{c}-27.95 * * * \\
(0.000)\end{array}$ & $\begin{array}{c}-27.95 * * * \\
(0.000)\end{array}$ \\
\hline $\mathrm{CHF}$ & 1 & $\begin{array}{c}-1.39 \\
(0.589)\end{array}$ & $\begin{array}{c}-1.53 \\
(0.521)\end{array}$ & 0 & $\begin{array}{c}-30.33 * * * \\
(0.000)\end{array}$ & $\begin{array}{c}-30.33 * * * \\
(0.000)\end{array}$ \\
\hline EUR & 1 & $\begin{array}{c}-1.85 \\
(0.354)\end{array}$ & $\begin{array}{c}-1.78 \\
(0.391)\end{array}$ & 0 & $\begin{array}{c}-27.22 * * * \\
(0.000)\end{array}$ & $\begin{array}{c}-27.22 * * * \\
(0.000)\end{array}$ \\
\hline GBP & 1 & $\begin{array}{c}-1.88 \\
(0.340)\end{array}$ & $\begin{array}{c}-1.88 \\
(0.343)\end{array}$ & 0 & $\begin{array}{c}-27.59 * * * \\
(0.000)\end{array}$ & $\begin{array}{c}-27.59 * * * \\
(0.000)\end{array}$ \\
\hline JPY & 1 & $\begin{array}{c}-1.52 \\
(0.524)\end{array}$ & $\begin{array}{c}-1.75 \\
(0.406)\end{array}$ & 0 & $\begin{array}{c}-30.57 * * * \\
(0.000)\end{array}$ & $\begin{array}{c}-30.57 * * * \\
(0.000)\end{array}$ \\
\hline
\end{tabular}

Given the stationarity in the first differences of the spot and forward rates, bivariate cointegration analysis shows a single long-run equilibrium with common stochastic trend, which provides a long-term co-movement, or tests the weak form of market efficiency hypothesis. Based on the initial computed AICs, the lag length is set equal to 4 in this analysis. Panels A, B, and C present the results of the cointegration over the entire sample, non-crisis, and crisis periods, respectively. The null hypothesis of no cointegration cannot be rejected over the entire sample period, except for the AUD. The statistical insignificance of test statistics implies that foreign exchange markets are 
not efficient over the period 2004-2010. However, The $\lambda_{\max }$ statistic for the AUD rejects the null hypothesis of one cointegrating vector.

The results over non-crisis and crisis periods are different from the entire sample. As shown in Panel B of Table 11, both $\lambda_{\text {trace }}$ and $\lambda_{\max }$ statistic values of the CAD, EUR, GBP, and JPY reject the first hypothesis of zero cointegrating vector, yet do not reject the second hypothesis of one cointegrating vector, showing that there exists at least one cointegrating vector in the system. Consequently, there exists a unique cointegrating vector between spot and forward exchange rates. The other currencies over the non-crisis period accept the null hypothesis of no cointegration.

The most interesting findings exist over the crisis period, as shown in Panel $\mathrm{C}$ of Table 11. The null hypothesis of no cointegration is rejected for all currencies, except for the AUD. Moreover, most currencies show that both $\lambda_{\text {trace }}$ and $\lambda_{\max }$ statistic values are able to reject the null hypothesis of zero cointegrating vector only, showing that there is only one cointegrating vector in the pair of currencies. The level of efficiency of the AUD does not follow the other currencies in this sample. 
Table 11 Bivariate Cointegration Rank Tests for Unbiased Forward Rate Hypothesis

This table reports the $\lambda_{\text {trace }}$ and $\lambda_{\max }$ test statistic from the Johansen cointegrating procedure. The null hypothesis indicates there are at most $r$ cointegrating vectors in the system. $r$ denotes the number of significant cointegrating vectors. The optimal lag length is from Schwarz information criterion (SIC).

Panel A: Entire Sample Period

\begin{tabular}{lccccc}
\hline Currency & $r \leq$ & $\lambda_{\text {Trace }}$ & $\begin{array}{c}5 \% \text { Critical } \\
\text { Value }\end{array}$ & $\lambda_{\text {Max }}$ & $\begin{array}{c}5 \% \text { Critical } \\
\text { Value }\end{array}$ \\
\hline AUD & 0 & 10.816 & 15.34 & 0.005 & 19.99 \\
& 1 & 1.720 & 3.84 & $11.220^{* *}$ & 9.13 \\
CAD & 0 & 10.582 & 15.34 & 11.781 & 19.99 \\
& 1 & 2.215 & 3.84 & 2.815 & 9.13 \\
CHF & 0 & 8.350 & 15.34 & 9.151 & 19.99 \\
& 1 & 0.303 & 3.84 & 0.896 & 9.13 \\
EUR & 0 & 12.505 & 15.34 & 12.617 & 19.99 \\
& 1 & 1.893 & 3.84 & 1.912 & 9.13 \\
GBP & 0 & 9.977 & 15.34 & 10.566 & 19.99 \\
& 1 & 2.610 & 3.84 & 2.707 & 9.13 \\
JPY & 0 & 9.509 & 15.34 & 10.288 & 19.99 \\
& 1 & 0.021 & 3.84 & 0.660 & 9.13 \\
\hline
\end{tabular}

Panel B: Non-Crisis Period

\begin{tabular}{lccccc}
\hline Currency & $r \leq$ & $\lambda_{\text {Trace }}$ & $\begin{array}{c}5 \% \text { Critical } \\
\text { Value }\end{array}$ & $\lambda_{\text {Max }}$ & $\begin{array}{c}5 \% \text { Critical } \\
\text { Value }\end{array}$ \\
\hline AUD & 0 & 9.632 & 15.34 & 9.996 & 19.99 \\
& 1 & 2.54 & 3.84 & 2.898 & 9.13 \\
CAD & 0 & $29.322^{* *}$ & 15.34 & $41.163^{* *}$ & 19.99 \\
& 1 & 1.745 & 3.84 & 3.554 & 9.13 \\
CHF & 0 & 7.005 & 15.34 & 7.744 & 19.99 \\
& 1 & 2.037 & 3.84 & 2.057 & 9.13 \\
EUR & 0 & $16.770^{* *}$ & 15.34 & 19.700 & 19.99 \\
& 1 & 0.617 & 3.84 & 1.187 & 9.13 \\
GBP & 0 & $17.820^{* *}$ & 15.34 & $21.086^{* *}$ & 19.99 \\
& 1 & 1.883 & 3.84 & 1.931 & 9.13 \\
JPY & 0 & $16.409^{* *}$ & 15.34 & $22.130^{* *}$ & 19.99 \\
& 1 & 5.787 & 3.84 & 8.054 & 9.13 \\
\hline
\end{tabular}


Panel C: Crisis Period

\begin{tabular}{lccccc}
\hline Currency & $r \leq$ & $\lambda_{\text {Trace }}$ & $\begin{array}{c}5 \% \text { Critical } \\
\text { Value }\end{array}$ & $\lambda_{\text {Max }}$ & $\begin{array}{c}5 \% \text { Critical } \\
\text { Value }\end{array}$ \\
\hline AUD & 0 & 14.208 & 15.34 & 14.448 & 19.99 \\
& 1 & 0.856 & 3.84 & 1.001 & 9.13 \\
CAD & 0 & $22.927^{* *}$ & 15.34 & $23.033^{* *}$ & 19.99 \\
& 1 & 2.137 & 3.84 & 2.213 & 9.13 \\
CHF & 0 & $29.874^{* *}$ & 15.34 & $30.477^{* *}$ & 19.99 \\
& 1 & 2.250 & 3.84 & 2.777 & 9.13 \\
EUR & 0 & $15.836^{* *}$ & 15.34 & 15.920 & 19.99 \\
& 1 & 2.392 & 3.84 & 2.410 & 9.13 \\
GBP & 0 & $25.517^{* *}$ & 15.34 & $26.049^{* *}$ & 19.99 \\
& 1 & 2.951 & 3.84 & 3.471 & 9.13 \\
JPY & $17.321^{* *}$ & 15.34 & 19.819 & 19.99 \\
& 1 & 1.67 & 3.84 & 2.784 & 9.13 \\
\hline
\end{tabular}

\subsection{Summary and Conclusions}

The tests of efficiency in the foreign exchange market have been the focus of this chapter. Several methodologies and their limitations are investigated. Using both spot exchange rates and their corresponding forward rates, one of the most traditional approaches to test the efficiency of a financial market is the theory of an unbiased forward predictor, which simply states that forward rate is an unbiased predictor of future spot rate under an efficient market. Based on daily data, the results show that foreign exchange markets are, in general, informationally inefficient, irrespective of time periods. However, the AUD market is least efficient in all periods. Moreover, the $\operatorname{GARCH}(1,1)$ results show that the foreign exchange market is time-varying. In general, the results from GARCH $(1,1)$ confirm previous findings on the efficiency of foreign exchange markets. Nevertheless, the results are mainly different over the crisis period in which the forward premiums on Switzerland, Euro, and Great Britain are weakly significant. The test of structural change between non-crisis and crisis periods is not significant, meaning that the environment of these two periods is not different. 
The cointegration method is one of the most popular techniques for market efficiency testing. The main results support the previous analysis. The null hypothesis of no cointegration cannot be rejected over the entire sample period, except for the AUD. The results over non-crisis and crisis periods are however different from the entire sample. Over the non-crisis period, only three currencies, the CAD, EUR, and GBP, are somewhat efficient. Interestingly, most currencies are more informationally inefficient over the crisis period, with the exception of the AUD. Using the cointegration method, the behavior of the AUD is unique from the other foreign exchange markets in terms of the level of the efficiency.

In conclusion, the inefficiency of the foreign exchange markets is detected in all periods, but this is not true for all cases. The results are different because of the methodologies and time periods considered when comparing with prior literature. The intuition of the methodology does affect the results. The econometric and statistical problems are also important issues for methodology selection. In sum, these findings are consistent with the mixed findings in foreign exchange markets. 


\section{CHAPTER 4: EFFICIENCY OF THE FOREX MARKET BEFORE AND DURING THE CRISIS (INTRADAY DATA)}

\subsection{Introduction}

Relying on the premise of the efficient market hypothesis, an equilibrium asset price should reflect completely and simultaneously all available information in the market. Price discovery provides a process or a mechanism to examine how, if at all, the markets subsume the information to reach an equilibrium asset price. In another words, price discovery may be regarded as the speed of the price adjustments for new information arrival.

Several studies have investigated the price discovery between different markets, for instance, between equity returns and their corresponding futures products (see, among others, Cheung and Ng (1990), Chan (1992), Fleming, Ostdiek, and Whaley (1996), Poskitt (2009), and De Boyrie, Pavlova, and Parhizgari (2012)). Though it is still unsettled, some researchers indicate that the futures markets' response to the new information arrival is quicker than that of the equity markets due to the requirement of a smaller capital margin (the so-called "leverage hypothesis"). However, there is no consensus in this regard. Some researchers have reached a contradictory position. For instance, some findings indicate that the options markets lag the spot markets due to the smaller trading costs in the spot markets (the so-called "trading cost hypothesis"). In general, when pairing two markets, the outcome seems to be open. For example, Fleming et al. (1996) show that S\&P 500 futures prices lead the S\&P 100 index put and call options. De Boyrie, Pavlova, and Parhizgari (2012) conclude that a mixed outcome for 
price discovery should be expected, since the underlying factors may not necessarily be all the same.

Within the context of these hypotheses and considering the rapidly growing importance of ETFs markets, our examination of price discovery between the ETFs (CurrencyShares) and spot exchange rates is expected to shed some light on the behavior of the currency ETFs in the AUD, CAD, CHF, EUR, GBP, and JPY during the crisis and non-crisis periods.

\subsection{Literature Review}

In a frictionless market, asset prices in different trading platforms or derivative prices on the same underlying assets are perfectly correlated; there exists no lead-lag relationship. Garbade and Silber (1979) introduce the meanings of "dominant" and "satellite" markets, in which a dominant market leads a satellite market only if the dominant market is more prominent in the price discovery mechanism, and the satellite market largely employs information from the dominant market. This is consistent with Glosten and Milgrom (1985) and Easley and O' Hara (1987), who show that informed trading in one particular market reveals new information arrival and subsequently leads prices on other markets.

Four theories exist that explain the role of informed traders in price discovery.

First, the leverage hypothesis states that the higher the debt-to-equity leverage security price, the greater the price discovery function. Informed traders prefer trading on high leverage securities due to higher return, given the arrival of new information. For example, Kawaller et al. (1987) study the relation between the S\&P 500 index and its 
futures and find that the futures prices lead cash prices by 20-45 minutes due to high leverage in the futures market. This supports the leverage hypothesis.

Second, the trading cost hypothesis. It states that a smaller trading cost induces informed trading. The higher net profit (deducted from the trading costs) encourages informed trading on a smaller trading cost security. For example, Kawaller et al. (1993) show that an increase in futures price volatility causes a stronger association between spot and futures markets. Due to a trading cost higher in spot than futures markets, the speed of information absorption in the two markets is different; arbitrageurs have no reward for executing orders in these two markets or take profits on the spot market, subsequently justifying trading in these two markets.

Third, the uptick rule hypothesis. It states that the short-selling in a security happens when the last change in a security price is non-negative. In practice, futures markets do not follow the uptick rule. Futures prices are more informationally efficient in a downturn market. Consequently, futures markets serve better price discovery function than spot markets.

Last, the marketwide information hypothesis. It states that an index or a basket of some securities for the objective of trading is a proxy on a particular subset of information. Subrahmanyam (1991) shows that liquidity traders are more efficient than other traders on index derivatives due to the well-diversified security-specific components of adverse selection of constituent securities in the index. Index derivatives support the processing of marketwide information. The function of price discovery, depending on the design of security, is whether or not the index is traded and reflected on marketwide information efficiently. 
Previous studies have examined price discovery with various scopes. First, a traditional approach to price discovery has been the study of the relation between equity and derivatives markets. Tse (1999) employs minute-by-minute data for the Dow Jones Industrial Index and its futures to investigate the price discovery process and finds that a futures market does better for the price discovery process. This finding confirms previous evidence on the leadership of the futures market (for example, $\mathrm{Ng}$ (1987), Herbst et al. (1987), Kawaller et al. (1987), and Chan (1992)). Ghosh (1993), employing a cointegration method, finds the leadership of a futures market in fifteen-minute prices, which is later confirmed by Wahab and Lashgari (1993), Dwyer et al. (1996), and Martens et al. (1982).

In sum, the evidence on the relation in the U.S. equity and futures markets mostly documents that a futures market has a better price discovery than its corresponding equity market. However, the evidence is not limited only to the U.S. International evidence on the relation is also investigated. ${ }^{3}$ Booth et al. (1999) study the role of price discovery between spot index and it index futures in Germany using transaction data and find similar results. Roope and Zurbruegg (2002) employ Taiwan Index Futures listed in two countries to investigate price discovery between the Singapore Exchange and Taiwan Futures Exchange. Both Hasbrouck (1995) as well as Gonzalo and Granger (1995) information share indicate that price discovery mainly starts from the Singapore futures market. So and Tse (2004) use three assets in Hong Kong for a discovery study: Hang

\footnotetext{
${ }^{3}$ For international studies, see, for example, Iihara et al. (1996) for Japan, Grünbichler et al. (1994) for Germany, Shyy et. al. (1996) for the France, Abhyankar (1995) for the U.K., De Jong and Donder (1998) for the Netherlands, Zhong et al. (2004) for Mexico, and Rittler (2012) for European Union.
} 
Seng index, Hang Seng index futures, and tracker fund (ETFs). Minute-by-minute results demonstrate that futures market is the most dominant asset.

The second area in the price discovery studies considers different trading platforms or system providers. Theissen (2002) compares the price discovery between open outcry and screen electronic trading platforms in the Frankfurt Stock Exchange. The finding of leadership of two systems is almost equally similar when using transaction data, but the screen trading system is better when using quote midpoints. The leadership of electronic trading system confirms previous findings by Grünbichler et al. (1994). Also, Stucki and Wasserfallen (1994) show the dominant role of equity markets over equity options markets. Poskitt (2009) compares two different electronic trading service providers on foreign exchange markets, namely GLOBEX and Reuter D3000. The finding explains why previous literature documents low information share for GLOBEX when return is computed from transaction prices. In general, Reuter D3000 leads GLOBEX.

Third area of the price discovery analyses has been in the commodities markets. Yang et al. (2001) use several types of U.S. agricultural futures contracts. They separate the assets on storability. This feature does not play any role in the price discovery mechanism. The cointegration results indicate that futures price is a biased estimate of cash price. Tse and Xiang (2005) study the energy futures market. They investigate the relation between regular futures and E-mini futures contracts on natural gas and crude oil. Although E-mini futures accounts for less than $1 \%$ of the volume of the regular futures in each asset, it contributes to price discovery significantly. The E-mini market has more than $30 \%$ information share generated by both Hasbrouck and Gonzalo and Granger 
methodologies. These findings are reaffirmed by Pavabutr and Chaihetphon (2010), who study the regular and mini gold futures contracts in the Multi Commodity Exchange of India. The daily results show the dominant role of mini gold futures with over $30 \%$ information share.

Fourth area of the price discovery analysis has been in the foreign exchange market. De Jong et al. (1998) study the efficiency of the Japanese yen/ Deutsch mark and implied exchange rate by Japanese yen/ United States dollar and Deutsch mark / United States dollar. The major price discovery function exists in direct quotes of Japanese yen/ Deutsch mark due to substantial noisy of the United States dollar. Tse et al. (2006), using both floor and electronic trading system for the Euro and Japanese yen futures markets, find that GLOBEX electronic trading facilitates most price discovery in the euro, and electronic trading is more efficient than floor trading. Rosenberg and Traub (2009) contradict most previous findings. They show that spot exchange market has larger information share than its corresponding futures markets. This finding is based on the argument that the time period study is not the same as in previous literature. Their most recent database carries more and different information in spot and futures exchange markets, and spot market is more transparent over this period.

Last and importantly, the latest approach is to investigate the role of the ETF market in price discovery function. The ETF market has become more important and has gained interest from investors during recent years. Tse and Erenburg (2003) employ the Nasdaq index and its ETF (QQQ) in different market exchanges, AMEX, NYSE, and ECNs (electronic communication networks). Using TAQ data from July 2 to August 31, 2001, price discovery starts from ECNs, NYSE, and AMEX, respectively. Other evidence 
by Tse et al. (2006) on the DJIA and its ETFs (DIAMOND), regular futures, and E-mini futures is also presented. The largest contribution in price discovery is E-mini futures market, followed by ETF market and regular futures market, respectively. Moreover, the electronic ETF trading platform (Archipelago: ArcaEX) is dominant in price discovery over the American Stock Exchange. Tse and Martinez (2007) extend previous research on ETF market by using prices and NAV of international iShares funds. The Hasbrouck information share indicates that iShares prices contribute more than iShares NAV.

In conclusion, there are some consistent evidences on price discovery. First, futures market leads its corresponding spot market, and spot market leads its corresponding options market. Second, electronic trading platforms contribute to price discovery greater than open outcry platforms. Third, E-mini futures contracts provide relatively high information share although the relative trading volume is tremendously lower than regular futures contracts. Last, the ETF market does provide some degree of price discovery function, albeit with inconclusive results at the present time.

\subsection{Methodology}

Two popular methods exist for the price discovery study, namely, the Gonzalo and Granger (1995) permanent-transitory model and Hasbrouck (1995) information share. These two venues show the contribution of the asset (market or trading platform) to the price discovery process and are generated from a vector error correction model (VECM). In this chapter, one of these methodologies is employed using ETFs of the currencies that we have so far analyzed. 
In order to study the role of the price discovery process between foreign exchange and currency ETF markets, Hasbrouck (1995)'s information share is employed for this study. ${ }^{4}$ The basic concept relies on the fact that when two time series are cointegrated, their price innovations share a common component. The information share is defined as the contribution of one market's innovation to the total innovation in the common efficient price.

Given that the first difference of the time series is usually stationary, an error correction model by Engle and Granger (1987) is presented as below:

$$
\Delta p_{t}=\mu+\sum_{k=1}^{K} \Gamma \Delta p_{t-k}+\alpha \beta^{\prime} p_{t-1}+\epsilon_{t}
$$

where $p_{t}=\left(F_{t} S_{t}\right)^{\prime}$ are the prices of currency ETF $\left(F_{t}\right)$ and spot exchange rate $\left(S_{t}\right)$ in each currency. $\mu, \alpha$, and $\Gamma$ are vectors of parameters with $(2 \times 1),(2 \times 1)$, and $(2 \times 2)$ dimensions, respectively. The Schwarz criterion is used to determine lag-length $K . \epsilon_{t}$ is the error vector with mean zero and $(2 \times 1)$ dimension and variance-covariance matrix $(\Omega) . \Delta$ is the difference operator and $\beta$ is the cointegrating vector.

A variant representation of a vector error correction model (VECM) may be stated as:

$$
\Delta F_{t}=a_{1}+\alpha_{1} z_{t-1}+\sum_{k=1}^{K} b_{E T F, k} \Delta F_{t-k}+\sum_{k=1}^{K} c_{E T F, k} \Delta S_{t-k}+\varepsilon_{E T F, k}
$$

\footnotetext{
${ }^{4}$ De Jong (2002) concludes that Hasbrouck (1995) information share is a more accurate estimator for the information generated in each asset. However, some studies show that the results from Gonzalo and Granger (1995) are qualitative similar to Hasbrouck information share (for example, Poskitt (2009)).
} 


$$
\Delta S_{t}=a_{2}+\alpha_{2} z_{t-1}+\sum_{k=1}^{K} b_{S p o t, k} \Delta F_{t-k}+\sum_{k=1}^{K} c_{S p o t, k} \Delta S_{t-k}+\varepsilon_{S p o t, k}
$$

where the error correction term is $z_{t-1}=F_{t-1}-S_{t-1}$. The VECM representation given in the above equations can be presented as a common trend representation model as:

$$
p_{t}=p_{0}+{ }_{\imath} C \sum_{i=1}^{t} \epsilon_{t-i}+C(L) \epsilon_{t}
$$

where $p_{0}$ is a constant vector. $l$ is column vector of ones. $C(L)$ is a matrix polynomial in the lag operator. $\imath C \sum_{i=1}^{t} \epsilon_{t-i}$ is the common random walk component for all prices, and $C(L) \epsilon_{t}$ is the transitory component with zero mean and stationary covariance.

Hasbrouck (1995) information share is the proportion of the innovations of market $j$ to the total innovation of the common efficient price. If the price innovations between markets are not correlated, the information share (IS) of the market $j$ is presented as:

$$
I S_{j}=\frac{c_{j}^{2} \Omega_{j j}}{c \Omega c^{\prime}}
$$

where $\mathbf{c}$ is the common row vector of the impact matrix in the common trend representation (See Hasbrouck (1995), page 1181). If the price innovations between markets are correlated, the information share (IS) of the market $j$ is presented as:

$$
I S_{j}=\frac{\left([c F]_{j}\right)^{2}}{c \Omega c^{\prime}}
$$

A Cholesky factorization of $\Omega$ is used to obtain the lower triangular matrix such that $\Omega=$ $F F^{\prime}$. The higher the information share, the larger the market impounding the new arrival of information. 


\subsection{Data}

Some details of the ETFs are in order at this point. CurrencyShares is an exchanged traded fund (ETF) in which the market price of a foreign currency is quoted at several prices in the United States dollar. ${ }^{5}$ The ETF shares are traded on the NYSE Arca. The purpose of this share is to reflect the price in the United States of the foreign currency. Thus, the prices of currency ETF and its corresponding spot rate should be largely similar. Hasbrouck (1995) suggests that there is an implicit efficient price in the cointegration system for the particular types of the asset in different markets. The difference in the implicit efficient price is due to each market-specific factor. Price discovery is defined as the innovations in common efficient price.

In this chapter our data are high frequency. Minute-by-minute data for the ETFs (currencyshares) and their associated spot exchange rates are from TradeStation. For consistency, weekends and holidays ${ }^{6}$ observations are excluded in the analysis, though the spot exchange market involves 24-hour trading. In addition, we truncate spot foreign

\footnotetext{
${ }^{5}$ According to the prospectus of currencyshares, "... each CurrencyShares Trust issues shares that represent units of fractional undivided beneficial interest in, and ownership of, the Trust. Shares may be purchased from each Trust only in one or more blocks of 50,000 Shares, called "Baskets". The Trusts issue shares in Baskets on a continuous basis to authorized participants (Depository Trust Company participants that are registered broker-dealers or other securities market participants, such as a bank or other financial institution, that are not required to register as a broker-dealer to engage in securities transactions, and that has entered into a Participant Agreement with the Trustee). It is expected that shares of each Trust will be offered and sold to the public by authorized participants at varying prices in U.S. Dollars to be determined by reference to, among other things, the market price of the currency held by the particular Trust and the trading price of the shares on the NYSE Arca at the time of each sale. Authorized Participants will not receive from the Trust, the Sponsor or any of their affiliates, any fee or other compensation in connection with the sale of shares, although they may receive commissions or fees from investors who purchase shares through their commission- or fee-based brokerage accounts." (www.currencyshares.com)

${ }^{6}$ There are 13 holidays for the entire sample data: Good Friday (April 6, 2007 and March 21, 2008), Memorial Day (May 28, 2007 and May 26, 2008), Independence Day (July 4, 2007 and July 4, 2008), Labor Day (September 3, 2007 and September 1, 2008), Thanksgiving Day (November 22, 2007), Christmas Day (December 25, 2007), New Year's Day (January 1, 2009), Martin Luther King Day (January 21, 2009), and President Day (February 18, 2009).
} 
exchange rate data, from 9.30 A.M. to 16.00 P.M. EST, which matches the operating hours in ETFs markets. The spot exchange rates are quoted in terms of United States dollars/ foreign currency in order to match with the currencyshares quotation. The currencyshares is the price in USD of the foreign currency. Due to the data limitation, the entire sample of this chapter starts April 1, 2007 and lasts through September 2008; the first nine months are referred to as the non-crisis period (April 1, 2007-December 31, 2007), and the second nine months are the crisis period (January 1, 2008-September 2008). The symbols for the different series are as follows:

\begin{tabular}{llll}
\hline \multicolumn{1}{c}{ Currency } & ETF & Spot Rate & ETF Inception Date \\
\hline Australian dollar & FXA & AUDUSD & June 21, 2006 \\
Canadian dollar & FXC & CADUSD & June 21, 2006 \\
Swiss franc & FXF & CHFUSD & December 9,2005 \\
Euro & FXE & EURUSD & June 21, 2006 \\
British pound & FXB & GBPUSD & June 21, 2006 \\
Japanese yen & FXY & JPYUSD & February 12, 2007 \\
\hline
\end{tabular}

\subsection{Empirical Results}

Given the stationarity of the variables in their first difference as shown in the previous chapter, we start our analysis by performing the vector error correction model (VECM). The equations (12) and (13) are employed for this purpose. The aim is to identify price discovery in the spot and ETFs markets. The number of optimal lag lengths is obtained by Schwarz Bayesian Criterion (SBC), which is set equal to 12 in most cases. The estimation of the VECM in this section is prerequisite to the computation of the information shares in the next section.

As an example of the above approach, Table 12 presents the results of the VECM estimation for the AUD/FXA. This computation is repeated for all days and for all pairs 
of currencies/ETFs. Because of the voluminous results of the VECM estimation, we present only the results of the first two lags in Table 12.

Table 12 Error Correction Model for Spot Exchange Rate and Currency Exchange Traded Fund

This table presents the results of a bivariate VECM for minute-by-minute AUD and FXA. Through an iterative process, this computation is repeated for all days and for all pairs of currencies/ETFs. In all cases, the optimal lag is obtained by Schwarz Bayesian Criterion (SBC), e.g., 12 lags for AUD/FXA in this example. Due to space limitation, this table presents only the first two lags. The $t$-statistics are shown in parentheses and $* * *$, and $* * *$ indicate significance at the $10 \%, 5 \%$, and $1 \%$, respectively.

\begin{tabular}{cccccc}
\hline & $\alpha$ & $\Delta A U D_{t-1}$ & $\Delta A U D_{t-2}$ & $\Delta F X A_{t-1}$ & $\Delta F X A_{t-2}$ \\
\hline AUD & -0.000 & 0.002 & 0.001 & -0.000 & 0.000 \\
& $(-0.36)$ & $(0.82)$ & $(0.47)$ & $(-0.66)$ & $(0.10)$ \\
FXA & $-0.002^{* * *}$ & $21.654^{* * *}$ & $16.556^{* * *}$ & $-0.168^{* * *}$ & $-0.152^{* * *}$ \\
& $(-14.57)$ & $(74.21)$ & $(55.83)$ & $(-67.12)$ & $(-59.94)$ \\
\hline
\end{tabular}

The error correction terms $(\alpha)$ of both the AUD and FXA are negative, and statistically significant for only FXA. This shows that an increase in the previous period error leads to a decrease in the current period FXA price. Further, the statistical insignificance of the negative error correction term of the AUD shows that this market does not seem to yield a clear response to the prior equilibrium error. The lag difference terms measure the short-run dynamics between the AUD and FXA. Considering the statistical significance of the estimated coefficients, the first two lag coefficients of the AUD influence the current FXA price, but those of the FXA do not influence the current AUD price.

Panels A, B, and C of Table 13 report the information share of spot exchange rate and currencyshares for the entire sample, non-crisis, and crisis periods, respectively. The results for the entire sample period (as shown in Panel A) show that spot exchange rates contribute up to $90 \%$ to the price discovery mechanism, and currencyshares ETFs 
contribute the rest. These estimates are based on the mid-point between the upper and lower bounds of information share. We anticipate that the significant contribution of spot exchange rates to the price discovery process is from the very active traders in foreign exchange markets, especially from institutional investors.

In conclusion, spot foreign exchange markets play a highly significant role in price discovery mechanisms, while ETFs provide a small contribution. In other words, spot exchange markets heavily lead ETFs markets. Our findings are the same for all currencies and ETFs.

Panels B and C of Table 13 present information share over the non-crisis and crisis periods, respectively. In general, the range between upper and lower bounds over the non-crisis period is narrower than that of the crisis period. The information shares of the spot exchange rates are lower over the crisis than non-crisis periods. However, it is not true for the AUD and CAD, whose information shares improve by approximately 0.06 over the crisis period. The EUR information share over the crisis period is smallest and shows the largest diminishing information share from the non-crisis period.

During the crisis period, the information shares of the spot currencies are relatively high, albeit diminishing, when compared with the entire sample and non-crisis periods. For the currency ETFs, the results show moderate gains. Most of the currency ETFs possess higher information shares over the crisis period, especially for the Euro (FXE) and Japanese yen (FXY). However, the information shares of the Australian dollar and Canadian dollar decline largely over the crisis period. In general, the currencies ETFs perform better over the crisis period, reflecting the potential that could have been available in the downside market. 
Table 13 Information Share of Spot Exchange Rates and Currency ETFs

This table presents Hasbrouck's (1995) information shares over different time periods, entire sample (Panel A), non-crisis (Panel B), and crisis (Panel C) periods. The currencyshares ETF is minute-by-minute trade price. The spot exchange rate is the mid-quote of its minute-by-minute bid and ask prices. Both upper and lower bounds are estimated for each day in the sample. The upper and lower bounds presented below are the average daily estimates. The average is the arithmetic mean of the paired upper and lower bounds presented below.

Panel A: Entire sample period (April 1, 2007 to September 30, 2008)

\begin{tabular}{lccc}
\hline Information Share & Upper Bound & Lower Bound & Average \\
\hline Currency & & & \\
AUD & 0.971 & 0.909 & 0.940 \\
CAD & 0.973 & 0.913 & 0.943 \\
CHF & 0.960 & 0.901 & 0.930 \\
EUR & 0.979 & 0.788 & 0.884 \\
GBP & 0.943 & 0.894 & 0.918 \\
JPY & 0.979 & 0.832 & 0.906 \\
& & & \\
Currencyshares & & & \\
FXA & 0.091 & 0.029 & 0.060 \\
FXC & 0.087 & 0.027 & 0.057 \\
FXF & 0.099 & 0.040 & 0.116 \\
FXE & 0.212 & 0.021 & 0.082 \\
FXB & 0.106 & 0.057 & 0.094 \\
FXY & 0.168 & 0.021 & \\
\hline
\end{tabular}

Panel B: Non-crisis period (April 1, 2007 to December 31, 2007)

\begin{tabular}{lccc}
\hline Information Share & Upper Bound & Lower Bound & Average \\
\hline Currency & & & \\
AUD & 0.963 & 0.922 & 0.943 \\
CAD & 0.964 & 0.919 & 0.942 \\
CHF & 0.941 & 0.904 & 0.922 \\
EUR & 0.976 & 0.908 & 0.942 \\
GBP & 0.913 & 0.872 & 0.892 \\
JPY & 0.980 & 0.888 & 0.934 \\
Currencyshares & & & \\
FXA & 0.078 & 0.037 & 0.057 \\
FXC & 0.081 & 0.036 & 0.058 \\
FXF & 0.096 & 0.059 & 0.078 \\
FXE & 0.092 & 0.024 & 0.058 \\
FXB & 0.128 & 0.087 & 0.108 \\
FXY & 0.112 & 0.020 & 0.066 \\
\hline
\end{tabular}

Panel C: Crisis period (January 1, 2008 to September 30, 2008) 


\begin{tabular}{lccc}
\hline Information Share & Upper Bound & Lower Bound & Average \\
\hline Currency & & & \\
AUD & 0.979 & 0.896 & 0.937 \\
CAD & 0.982 & 0.907 & 0.944 \\
CHF & 0.980 & 0.897 & 0.939 \\
EUR & 0.982 & 0.668 & 0.825 \\
GBP & 0.973 & 0.916 & 0.945 \\
JPY & 0.978 & 0.777 & 0.877 \\
& & & \\
Currencyshares & & & \\
FXA & 0.104 & 0.021 & 0.063 \\
FXC & 0.093 & 0.018 & 0.056 \\
FXF & 0.103 & 0.020 & 0.061 \\
FXE & 0.332 & 0.018 & 0.175 \\
FXB & 0.084 & 0.027 & 0.055 \\
FXY & 0.223 & 0.022 & 0.123 \\
\hline
\end{tabular}

We further investigate the difference of market leadership between non-crisis and crisis periods. Table 14 presents the results of the paired $t$-test values of information shares between the non-crisis and crisis periods. Most of the $t$-values of the average values are statistically significant, showing that leadership of the instruments is statistically changed. More specifically, the leadership of the EUR and JPY is statistically decreasing over the crisis period, while that of the CHF and GBP is statistically increasing. In another words, their corresponding ETFs do worse for the EUR and JPY and do better for the CHF and GBP in price discovery process over the crisis period. Note that the results of the currencyshares are a mirror image of their corresponding spot exchange rates, albeit opposite by design. 
Table 14 Test of Equality of Information Share Between the Non-Crisis and Crisis Periods

This table presents paired $t$-statistic values for information shares over the non-crisis and crisis periods. ${ }^{*}, * *$, and $* * *$ are significant at the $10 \%, 5 \%$, and $1 \%$, respectively.

\begin{tabular}{lrrr}
\hline Information Share & Upper Bound & Lower Bound & Average \\
\hline Currency & & & \\
AUD & $-2.56^{* *}$ & $2.74^{* * *}$ & 0.69 \\
CAD & $-3.01^{* * *}$ & 1.35 & -0.38 \\
CHF & $-5.16^{* * *}$ & 0.61 & $-1.97^{* *}$ \\
EUR & $-1.73^{*}$ & $14.53^{* * *}$ & $12.88^{* * *}$ \\
GBP & $-5.64^{* * *}$ & $-3.47^{* * *}$ & $-4.59^{* * *}$ \\
JPY & 0.46 & $8.71^{* * *}$ & $7.63^{* * *}$ \\
& & & \\
Currencyshares & & & -0.69 \\
FXA & $-2.74^{* * *}$ & $2.56^{* *}$ & 0.38 \\
FXC & -1.35 & $3.01^{* * *}$ & $1.97 * *$ \\
FXF & -0.61 & $5.16^{* * *}$ & $-12.88^{* * *}$ \\
FXE & $-14.53^{* * *}$ & $1.73^{* *}$ & $4.59^{* * *}$ \\
FXB & $3.47^{* *}$ & $5.64^{* * *}$ & $-7.63 * * *$ \\
FXY & $-8.71^{* * *}$ & -0.46 & \\
\hline
\end{tabular}

4.5 Summary and Conclusions

This chapter examines the efficiency of the foreign exchange markets using intraday data. Minute-by-minute data is employed for the analysis. Spot exchange rates and their corresponding ETFs (currenyshares) are the sample data. The efficiency of the foreign exchange markets is studied through price discovery mechanism. The results for the entire sample period show that spot exchange markets make a greater contribution to the price discovery, while ETFs play a small role. Thus, the spot foreign exchange market leads the ETF market significantly. The results of both non-crisis and crisis periods confirm the same evidence, though they are not identical in the degree of contributions.

The comparison of information shares over the non-crisis and crisis periods confirms the superior role of foreign exchange rates over their corresponding currency 
ETFs. In both periods the leadership of the foreign exchange is in place, though not the same in the two periods. A few of the spot currencies show superior information shares over the crisis period. Interestingly, most of the information shares of the currency ETFs over the crisis period are significantly larger than those of the non-crisis period. Another interpretation is that the spot exchange market is more transparent and that spot prices can incorporate new information efficiently when the markets are more stable. The volatility factor may have played a role in the price discovery process, but it is the subject of a separate study. The small information share of the currency ETFs points out the roles of hedging instruments and a passive indexing that could be employed in these markets. 


\section{CHAPTER 5: FINAL SUMMARY AND CONCLUSIONS}

\subsection{Summary of Empirical Findings}

This dissertation investigates the behaviors of the foreign exchange market in the most recent financial crisis. The financial crisis of 2008 is different from prior financial crises (for example, Asian crisis in 1997 and Russian crisis in 1998). The 2008 crisis is unique, global, and severe. The objectives of this dissertation are: 1) to examine the behaviors of the foreign exchange market, 2) to compare the behaviors of the foreign exchange market between non-crisis and crisis periods, 3) to test the efficiency of the foreign exchange market, and 4) to compare the efficiency of the foreign exchange market between non-crisis and crisis periods

This dissertation begins with the exploration of fundamental characteristics of foreign exchange markets using daily data. We have designed and presented the analysis in three parts: entire sample, non-crisis, and crisis periods. The break point of two periods is determined by the NBER official announcement, which is an ex post date. The data include six major actively trading forward and spot foreign exchange rates, namely, the Australian dollar, Canadian dollar, Swiss franc, Euro, British pound, and Japanese yen. All values are expressed as foreign currency per United States dollar, or indirect quotations in view of American investors. Chapter 4 further expands the analysis by including the currency ETFs (CurrencyShares) corresponding with the currencies in the prior Chapters. High frequency data is employed to investigate price discovery mechanisms between spot foreign exchange and currency ETF markets.

The second chapter of this dissertation provides basic characteristics of both spot and forward exchange rates for the three different subsamples: entire data, non-crisis, and 
crisis periods. Further, we have investigated seasonal patterns over each period. In general, basic statistics of the spot and corresponding forward markets are similar. However, the currencies' spot and their corresponding forward rates are more diverse over the non-crisis period, with the exception of EUR and GBP. The USD is weakened during the crisis period, creating substantially deviations across all currencies. The results indicate that the behavior of basic statistics over the non-crisis period is significantly different from the crisis period. All spot and forward exchange rates strongly reject the null hypothesis of normal distribution in all periods. The null hypothesis of the equality of means between the non-crisis and crisis periods is strongly rejected using parametric (paired t-test) as well as nonparametric (Wilcoxon signed test) statistical tests. However, the Wilcoxon signed test cannot reject the equality of means for the GBP forward rate.

The behavior of each currency for the days of the week is also investigated. The results show varied situations. Most currencies over the full sample period are most dispersed on Mondays and Fridays. The CAD and EUR are most volatile on Wednesdays. The spreads in forward markets are wider than in corresponding spot markets and are relatively constant over the entire week. The standard deviations on each day of forward markets are minimally lower than those of spot markets over the crisis period, but slightly higher over the non-crisis period. In addition, the behaviors of higher moments of spot and forward markets during the non-crisis and crisis periods are inconclusive. The behaviors of spreads in these two periods are not significantly different from those of the full sample period. The spreads in forward markets are still larger than in corresponding spot markets. 
Examining further dynamics of the currencies, the results indicate that the currencies' spot and forward markets are more dispersed at the beginning (the AUD, CAD, and CHF) and end (the JPY) of a year. However, the EUR are most dispersed in the middle of a year. In sum, the higher moment factors of all currencies are timevarying, behave differently from month to month, and are consistent with the results of the end-of-the week effect. However, the spreads for both spot and forward rates are relatively invariant over a year; thus they are not seasonal. It is also important to note that the size of the spread at the end of the month is almost identical with that of the end of the week.

In general, the volatility pattern computed from the squared returns of spot currencies is relatively stable over a weekly interval, with the exception of the JPY; however, each currency possesses a unique volatility style. For monthly intervals, the currencies' spot markets at the beginning and end of a year are more volatile than the other months, with the exception of the CAD. The volatility pattern over a monthly interval still displays differently from currency to currency: two-humped for the AUD, CAD, and EUR, W-shaped for the CHF, three-humped for the GBP, and flat U-shaped for the JPY.

The third chapter of this dissertation examines the efficiency of the foreign exchange market employing daily data. Several methodologies are considered and results are discussed in detail. Starting with a famous well-known efficiency test in foreign exchange markets, i.e., the forward rate as an unbiased predictor of future spot rate, the results are presented in terms of both level and percentage change in currency prices. The results in the level regression model show that the model is well fitted, providing high R- 
square value, and though the theory of unbiased forward predictor may be argued to hold to some extent, the estimated results suffer from serious econometric shortcomings. The nonstationarity of the exchange rate variables in their levels is a major shortcoming. Moreover, the Durbin-Watson autocorrelation statistic values are all too small, signaling other econometric problems. Thus, the results obtained by using level of variables are not well suited. To rectify these problems, the results from the percentage change in variables are computed. The outcome does not support that forward rate is an unbiased predictor of future spot rate in nearly all cases. The AUD market is the least informationally efficient in this sample.

As a third methodology, GARCH $(1,1)$ specification model is employed. This effort shows that the foreign exchange market is time-varying. In particular, the CHF, EUR, and GBP over the crisis period are not informationally efficient. The rest exhibit nearly the same results.

As an extension of the above methodologies and experiments, we examine whether the structure of the models remains the same for the non-crisis and crisis periods. We perform statistical tests over these two periods and find that there is no difference between these two periods, except for the CHF.

As the last item in Chapter 3, one of the most popular techniques in efficiency testing, i.e., cointegration methodology, is employed. Bivariate cointegration between spot and its forward rates in each currency shows that the null hypothesis of no cointegration cannot be rejected over the entire sample period in all cases. But the results are not true for the sub-samples. In most cases over the non-crisis period the null hypothesis, except for the AUD and CHF, is rejected. A similar pattern occurs over the 
crisis period as well. Only for AUD the null hypothesis cannot be rejected. In sum, the evidence on the efficiency of the foreign market is not supported. In some instances the outcome is mixed, largely depending upon the methodology and time period. Our findings confirm previous mixed results in previous studies.

Chapter 4 focuses on the informational efficiency in the foreign exchange market under a completely different framework. High frequency data is employed throughout this chapter. We extend our analyses of foreign exchange markets by using currency ETFs (CurrencyShares) and their corresponding spot exchange rates. The currencies in this chapter are the same as in Chapters 2 and 3. The currency ETF is the price of the foreign currency in USD. The focus in this chapter is on price discovery, which can be interpreted as the efficiency. We employ the Hasbrouck (1995) information share to detect the price discovery function between the spot and ETF markets. The larger the information share, the quicker is the market in impounding the new arrival of information. Our findings show that the price discovery largely exists in the spot market. The ETF market lags spot market significantly. These findings are consistent across all cases.

The empirical findings of this dissertation have important implications for traders, importers and exporters, financial managers, and portfolio managers in selecting currency investment and seeking arbitrage profits in the foreign exchange market. The findings are also expected to contribute to the current knowledge that the currency traders and policy makers often rely upon in world-wide currency management. The results reported in this study suggest that arbitrage opportunities exist and arbitrageurs can gain profit from the disparity. 


\subsection{Limitations}

One of the common limitations of the efficiency testing is the nature of the data that enters the econometric models. Although there are some widely acceptable methodologies to account for this factor, the assumptions underlying the models are not always well specified. For example, some researchers argue fractional cointegration is the best method for efficiency testing. Another issue is the transparency of the data. When currencies are traded over the counter, then the quality of the data is a suspect. Though there are many data collectors in the marketplace nowadays, there is no consensus what data source is the best. Some papers argue their superior results from the better and more accurate database. Finally, the existence of time zones and matching data collection across them properly are also of some concern, particularly in the global currency markets that operate 24 hours.

\subsection{Future Research}

Further research on the test of efficiency in the foreign exchange market could focus on the new methodologies, for example spectral analysis or compression algorithms. Due to the lack of comprehensive research in these areas, it is not clear if these methodologies will be able to generate more accurate results in the foreign exchange market.

The variety and availability of the data in the foreign currency market should also be noted. This feature renders research on currency with relative ease and provides the potential for more complex analysis. Finally, the emerging markets are another area for

further research. Infrequent trading and some other factors such as market fragility and 
unstable political climate in these markets warrant further analysis. There is also an untested area when the spot exchange market and its corresponding FX currency interest swap market is considered. These are all potentials for future research. 


\section{REFERENCES}

Abhyankar, A. H., (1995). Return and volatility dynamics in the FT-SE 100 stock index and stock index futures markets. Journal of Futures Markets, 15:457-488.

Aroskar, R., Sarkar, S. K., and Swanson, P. E., (2004). European foreign exchange market efficiency: Evidence based on crisis and noncrisis periods. International Review of Financial Analysis, 13:333-347.

Balillie, R. T. and Bollerslev, T., (1989). Common stochastic trends in a system of exchange rates. Journal of Finance, 44:167-181.

Balillie, R. T. and Bollerslev, T., (1994). Cointegration, fractional cointegration, and the exchange rate dynamics. Journal of Finance, 49:737-745.

Barkoulas, J. and Buam, C. F., (1997). A re-examination of the fragility of evidence from cointegration-based tests of foreign exchange market efficiency. Applied Financial Economics, 7:635-643.

Booth, G. G., So, R. W., and Tse, Y., (1999). Price discovery in the German equity index and derivatives markets. Journal of Futures Markets, 6:619-643.

Chan, K., (1992). A further analysis of the lead-lag relationship between the cash market and stock futures market. Review of Financial Studies, 5:123-152.

Cheung, Y. W. and Ng, L. K., (1990). The dynamics of S\&P 500 index and S\&P 500 futures intraday price volatilities. Review of Futures Markets, 2:458-486.

Christodoulakis, N. M. and Kalyvitis, S. C. (1997). Efficiency testing revisited: A foreign exchange market with Bayesian learning. Journal of International Money and Finance, $16: 367-385$.

Crowder, W. J., (1994). Foreign exchange market efficiency and common stochastic trends. Journal of International Money and Finance, 13:551-564.

Crowder, W. J., (1996). A note on cointegration and international capital market efficiency: A reply. Journal of International Money and Finance, 15:661-664.

De Boyrie, M. E., Pavlova, I., and Parhizgari, A. M., (2012). Price discovery in currency markets: Evidence from three emerging markets. FIU manuscript.

De Jong, F. and Donders, M. W. M., (1998). Intraday lead-lag relationships between futures-options, and stock market. European Financial Review, 1:337-359.

De Jong, F., Mahieu, R., and Schotman, P., (1998). Price discovery in the foreign exchange market: An empirical analysis of the yen/dmark rate. Journal of International Money and Finance, 17:5-27. 
De Jong, F., 2002. Measures of contribution of price discovery: A comparison. Journal of Financial Markets, 5:323-328.

Diebold, F. X., Gardeazabal, J., and Yilmaz, K., (1994). On cointegration and exchange rate dynamics. Journal of Finance, 49:727-735.

Douglas, J. and Rahman, A., (1986). Foreign currency futures and monetary policy announcements: An intervention analysis. Journal of Futures Market, 6:343-373.

Dwyer, G. P. J., Locke, P., and Yu, W., (1996). Index arbitrage and nonlinear dynamics between the S\&P 500 futures and cash. Review of Financial Studies, 9:301-332.

Dwyer, G. P. J. and Wallace, M. S., (1992). Cointegration and market efficiency. Journal of International Money and Finance, 11:318-327.

Easley, D. and O'Hara, M., (1987). Price, trade size, and information in securities markets. Journal of Financial Economics, 19:69-90.

Enders, W., Applied Econometric Time Series 2nd Ed.

Engle, R. F. and Granger, C. W. J., (1987). Co-integration and error correction: Representation, estimation and testing. Econometrica, 55:251-276.

Fama, E. F., (1970). Efficient capital markets: A review of theory and empirical work. Journal of Finance, 25:383-417.

Fleming, J., Ostdiek, B., and Whaley, R. E., (1996). Trading costs and the relative rates of price discovery in stock, futures and option markets. Journal of Futures Markets, 16:353-387.

Garbade, K.D. and Silber, W.L., (1979). Dominant and satellite markets: A study of dually traded securities. Review of Economics and Statistics, 60:455-460.

Ghosh, A., (1993). Cointegration and error correction models: Intertemporal causality between index and futures prices. Journal of Futures Markets, 13:193-198.

Glosten, L. R. and Milgrom, P. R., (1985). Bid, ask and transaction prices in a specialist market with heterogeneously informed traders. Journal of Financial Economics, 14:71-100.

Gonzalo, J. and Granger, C. W. J., (1995). Estimation of common long-memory components in cointegrated systems. Journal of Business and Economic Statistics, $13: 27-35$.

Greene, W. H., (2003). Econometric Analysis 5th Ed. 
Guillaume, D. M., Dacorogna, M. M., Davé, R. R., Müller, U. A., Olsen, R. B., and Pictet, O. V., (1997). From the bird's eye to the microscope: A survey of new stylized facts of the intra-daily foreign exchange markets. Finance and Stochastics, 1:95-129.

Grünbichler, A., Longstaff, F. A., and Schwartz, E., (1994). Electronic screen trading and the transmission of information: An empirical examination. Journal of Financial Intermediation, 3:166-187.

Hakkio, C. S. and Rush, M., (1989). Market efficiency and cointegration: An application to the sterling and deutschmark exchange markets. Journal of International Money and Finance, 8:75-88.

Hasbrouck, J., (1995). One security, many markets: Determining the contributions to price discovery. Journal of Finance, 50:1175-1199.

Haug, A. A., MacKinnon, J. G., and Michelis, L., (2000). European monetary union: A cointegration analysis. Journal of International Money and Finance, 19:419-432.

Herbst, A., McCormack, J., and West, E., (1987). Investigation of a lead-lag relationship between spot indices and their futures contracts. Journal of Futures Markets, 7:373-382.

$\mathrm{Hu}, \mathrm{X}$. , (1997). Macroeconomic uncertainty and the risk premium in the foreign exchange market. Journal of International Money and Finance, 16:699-718.

Iihara, Y., Kato, K., and Tokunaga, T., (1996). Intraday return dynamics between the cash and futures markets in Japan. Journal of Futures Markets, 16:147-162.

Johansen, S., (1988). Statistical analysis of cointegration vectors. Journal of Economic Dynamics and Control, 12:231-254.

Johansen, S., (1991). Estimation and hypothesis testing of cointegration vectors in Gaussian vector autoregressive models, Econometrica, 59:1551-1580.

Johansen, S. and Jeselius, K., (1990). Maximum likelihood estimation and inference on cointegration-with applications to the demand for money. Oxford Bulletin of Economics and Statistics, 52:169-210.

Kawaller, I. G., Koch, P. D., and Koch, T. W., (1987). The temporal price relationship between S\&P 500 futures and the S\&P 500 index. Journal of Finance, 42:1309-1329.

Kawaller, I. G., Koch, P. D., and Koch, T. W., (1993). Intraday market behavior and the extent of feedback between S\&P 500 futures prices and the S\&P 500 index. Journal of Financial Research, 14:107-121.

Kühl, M., (2010). Bivariate cointegration of major exchange rates, cross-market efficiency and the introduction of the Euro. Journal of Economics and Business, 62:1-19. 
Labonte, M., (2009). U.S. economy in recession: Similarities to and differences from the past. Congressional Research Service.

Lajaunie, J. P. and Naka, A., (1992). Is the Tokyo spot foreign exchange market consistent with the efficient market hypothesis?. Review of Financial Economics, 68:74.

Lajaunie, J. P., McManis, B., and Naka, A., (1996). Further evidence on foreign exchange market efficiency: An application of cointegration tests. Financial Review, 31:553-564.

Ligeralde, A. V., (1997). Covariance matrix estimators and tests of market efficiency. Journal of International Money and Finance, 16:323-343.

Liu, C. Y. and He, J., (1992). Risk premia in foreign currency futures. Financial Review, 27:571-587.

MacDonald, R. and Taylor, M. P., (1989). Foreign exchange market efficiency and cointegration: Some evidence from the recent float. Economics Letters, 29:63-68.

Maddala, G. S., Introduction to Economics 3rd Ed.

Martens, M., (1998). Price discovery in high and low volatility periods: Open outcry versus electronic trading. Journal of International Financial Markets, Institutional and Money, 8:243-260.

Martens, M., Kofman, P., and Vorst, T. C. F., (1998). A threshold error-correction model for intraday futures and index returns. Journal of Applied Econometrics, 13:245-263.

Melvin, M. and Taylor, M. P., (2009). The crisis in the foreign exchange market. CESIFO Working Paper No. 2707.

$\mathrm{Ng}$, N., (1987). Detecting spot forecast in futures prices using causality tests. Review of Futures Markets, 6:250-267.

Pavabutr, P. and Chaihetphon, P., (2010). Price discovery in the Indian gold futures market. Journal of Economics and Finance, 34:455-467.

Poskitt, R., (2009). Price discovery in electronic foreign exchange markets: The sterling/dollar market. Journal of Futures Markets, 30:590-606.

Rangvid, J. and Sørensen, C., (2002). Convergence in the ERM and declining numbers of common stochastic trends. Journal of Emerging Market Finance, 1:183-213.

Rittler, D., (2012). Price discovery and volatility spillovers in the European Union emissions trading scheme: A high-frequency analysis. Journal of Banking and Finance, 36:774-785. 
Roope, M. and Zurbruegg, R., (2002). The intra-day price discovery process between the Singapore exchange and Taiwan futures exchange. Journal of Futures Markets, 22:219-240.

Rosenberg, J. V. and Traub, L. G., (2009). Price discovery in the foreign currency futures and spot market. Journal of Derivatives, 17:7-25.

Sephton, P. S. and Larsen, H. K., (1991). Tests of exchange market efficiency: Fragile evidence from cointegration tests. Journal of International Money and Finance, 10:561-570.

Shyy, G., Vijayraghavan, V., and Scott-Quinn, B., (1996). A further investigation of the lead-lag relationship between the cash market and the stock index futures market with the use of bid/ask quotes: The case of France. Journal of Futures Markets, 16:405-420.

So, R. W. and Tse, Y., (2004). Price discovery in the Hang Seng index markets: Index, futures, and the tracker fund. Journal of Futures Markets, 24:887-907.

Stephan, J. A. and Whaley, R. E., (1990). Intraday price change and trading volume relations in the stock and stock option markets. Journal of Finance, 45:191-220.

Stoll, H. R. and Whaley, R. E., (1990). The dynamics of stock index and stock index futures returns. Journal of Financial and Quantitative Analysis, 25:441-468.

Stucki, T. and Wasserfallen, W., (1994). Stock and option markets: the Swiss evidence. Journal of Banking and Finance, 18:881-893.

Subrahmanyam, A., (1991). A theory of trading in stock index futures. Review of Financial Studies, 4:17-51.

Theissen, E., (2002). Price discovery in floor and screen trading systems. Journal of Empirical Finance, 9:455-474.

Tse, Y., (1999). Price discovery and volatility spillovers in the DJIA index and futures markets. Journal of Futures Markets, 19:911-930.

Tse, Y. and Erenburg, G., (2003). Competition for order flow, market quality, and price discovery in the Nasdaq 100 index tracking stock. Journal of Financial Research, 26:301-318.

Tse, Y. and Martinez, V., (2007). Price discovery and informational efficiency of international iShares funds. Global Finance Journal, 18:1-15.

Tse, Y. and Xiang, J., (2005). Market quality and price discovery: Introduction of the E-mini energy futures. Global Finance Journal, 16:164-179. 
Tse, Y., Bandyopadhyay, P., and Shen, Y, P., (2006). Intraday price discovery in the DJIA index markets. Journal of Business Finance and Accounting, 33:1572-1585.

Tse, Y., Xiang, J., and Fung, J. K., (2006). Price discovery in the foreign exchange futures market. Journal of Futures Markets, 26:1131-1143.

Wahab, M. and Lashgari, M., (1993). Price dynamics and error correction in stock index and stock index futures markets: A cointegration approach. Journal of Futures Markets, 13:711-742.

Woo, K. Y., (1999). Cointegration analysis of intensity of the ERM currencies under the European Monetary System. Journal of International Financial Markets, Institutions and Money, 9:393-405.

Yang, J., Bessler, D. A., and Leatham, D. J., (2001). Asset storability and price discovery in commodity futures markets: A new look. Journal of Futures Markets, 21:279-300.

Zhong, M., Darrat, A. F., and Otero, R., (2004). Price discovery and volatility spillovers in index futures markets: Some evidence from Mexico. Journal of Banking and Finance, 28:3037-3054. 
VITA

\section{CHAIYUTH PADUNGSAKSAWASDI}

May 11, 1979

2001

2006

2007-2011
Born, Bangkok, Thailand

B.S., Finance

Thammasat University

Bangkok, Thailand

M.S., Finance

Florida International University

Miami, Florida

Graduate Teaching and

Research Assistant

Florida International University

Miami, Florida

\section{PUBLICATIONS AND PRESENTATIONS}

"Bankruptcy Prediction of Real Estate Firms in Thailand," with Suporn Treewichayapong and Pornchai Chunhachinda, The International Journal of Finance, forthcoming.

"An Analysis of Credit Default Swaps: 2002-2009 Period," with Ali M. Parhizgari, Proceedings of the 11th ISINI Conference: New Challenges, New Methodologies.

"The Return-Implied Volatility Relation for Commodity ETFs: A Behavioral Perspective," Midwest Finance Association Meeting, Chicago, Illinois 2011.

"Alpha: Empirical Evidence of Mutual Fund Performance under Different Economic Cycles and over Fund Objectives," Eastern Finance Association Meeting, Savannah, Georgia 2011.

"Bankruptcy Prediction of Real Estate Firms in Thailand," Multinational Finance Society, Rome, Italy 2011. 
"An Analysis of Credit Default Swaps: 2002-2009 Period," Global Finance Conference, Bangkok, Thailand 2011 and the 11th ISINI Conference: New Challenges, New Methodologies, Hermosillo, Sonora, Mexico 2011.

"ETFs in Emerging Markets: Price Discovery through Volatility," Global Finance Conference, Chicago, Illinois 2012. 